\title{
Inversion of adjunction for quotient singularities
}

\author{
Yusuke Nakamura and Kohsuke Shibata
}

\begin{abstract}
We prove the precise inversion of the adjunction formula for quotient singularities and klt Cartier divisors. As an application, we prove the semi-continuity of minimal log discrepancies for klt hyperquotient singularities.
\end{abstract}

\section{Introduction}

The minimal log discrepancy is an invariant of singularities defined in birational geometry. Shokurov proved that two conjectures on the minimal log discrepancies, the LSC (lower semicontinuity) conjecture and the ACC (ascending chain condition) conjecture, imply the termination of flips conjecture [Sho04]. We refer the reader to [Kaw14, Kaw15, Nak16a, MN18, Kaw21, HLS19] for the recent developments related to the ACC conjecture. In this paper, we focus on the LSC conjecture. We always work over an algebraically closed field of characteristic zero unless otherwise stated.

Conjecture 1.1 (LSC conjecture). Let $(X, \mathfrak{a})$ be a log pair, and let $|X|$ be the set of all closed points of $X$ with the Zariski topology. Then the function

$$
|X| \rightarrow \mathbb{R}_{\geqslant 0} \cup\{-\infty\}, \quad x \mapsto \operatorname{mld}_{x}(X, \mathfrak{a})
$$

is lower semi-continuous.

The LSC conjecture is known to be true in the following cases:

(1.1.1) the case when $\operatorname{dim} X \leqslant 3$ [Amb99],

(1.1.2) the case when $X$ is smooth [EMY03],

(1.1.3) more generally, the case when $X$ is a normal local complete intersection variety [EM04],

(1.1.4) the case when $X$ has only quotient singularities [Nak16b],

(1.1.5) the case when $X$ is a smooth variety over an algebraically closed field of arbitrary characteristic under some condition [Shi19].

The main purpose of this paper is to prove the LSC conjecture for varieties with hyperquotient singularities, more generally, the quotient of a complete intersection variety by a finite linear group action.

Received 30 November 2020, accepted in final form 30 July 2021.

2020 Mathematics Subject Classification 14E18 (primary), 14E30, 14B05 (secondary).

Keywords: minimal log discrepancy, arc space, hyperquotient singularity.

This journal is (C) Foundation Compositio Mathematica 2022. This article is distributed with Open Access under the terms of the Creative Commons Attribution Non-Commercial License, which permits non-commercial reuse, distribution, and reproduction in any medium, provided that the original work is properly cited. For commercial re-use, please contact the Foundation Compositio Mathematica.

The first author is partially supported by the Grant-in-Aid for Young Scientists (KAKENHI No. 18K13384).

The second author is partially supported by the Grant-in-Aid for Young Scientists (KAKENHI No. 19K14496). 
ThEOREM 1.2 (Theorem 6.2). Suppose that a finite subgroup $G \subset \mathrm{GL}_{N}(k)$ acts on $\mathbb{A}_{k}^{N}$ freely in codimension one. Let $X:=\mathbb{A}_{k}^{N} / G$ be the quotient variety. Let $Y$ be a subvariety of $X$ of codimension $c$ which has only Kawamata log terminal (klt) singularities, and let $\mathfrak{a}$ be an $\mathbb{R}$-ideal sheaf on $Y$. Suppose that $Y$ is locally defined by $c$ equations in $X$. Then the function

$$
|Y| \rightarrow \mathbb{R}_{\geqslant 0} \cup\{-\infty\}, \quad y \mapsto \operatorname{mld}_{y}(Y, \mathfrak{a})
$$

is lower semi-continuous, where we denote by $|Y|$ the set of all closed points of $Y$ with the Zariski topology.

In this paper, we also treat the PIA (precise inversion of adjunction) conjecture.

Conjecture 1.3 (PIA conjecture, [Kol92, Conjecture 17.3.1]). Let $(X, \mathfrak{a})$ be a log pair, and let $D$ be a normal Cartier prime divisor. Let $x \in D$ be a closed point. Suppose that $D$ is not contained in the cosupport of the $\mathbb{R}$-ideal sheaf $\mathfrak{a}$. Then we have

$$
\operatorname{mld}_{x}\left(X, \mathfrak{a} \mathcal{O}_{X}(-D)\right)=\operatorname{mld}_{x}\left(D, \mathfrak{a} \mathcal{O}_{D}\right) .
$$

The PIA conjecture is known to be true in the following cases:

(1.3.1) the case when $X$ is smooth [EMY03],

(1.3.2) more generally, the case when $X$ is a normal local complete intersection variety [EM04].

In this paper, we study the PIA conjecture for varieties with quotient singularities.

Theorem 1.4 (= Corollary 6.1). Suppose that a finite subgroup $G \subset \mathrm{GL}_{N}(k)$ acts on $\mathbb{A}_{k}^{N}$ freely in codimension one. Let $X:=\mathbb{A}_{k}^{N} / G$ be the quotient variety, and let $x \in X$ be the image of the origin of $\mathbb{A}_{k}^{N}$. Let $Y$ be a subvariety of $X$ through $x$ of codimension $c$, and let $\mathfrak{a}$ be an $\mathbb{R}$-ideal sheaf on $Y$. Suppose that $Y$ is locally defined by $c$ equations at $x$ in $X$. Let $D$ be a Cartier prime divisor on $Y$ through $x$ with a klt singularity at $x \in D$. Suppose that $D$ is not contained in the cosupport of the $\mathbb{R}$-ideal sheaf $\mathfrak{a}$. Then it follows that

$$
\operatorname{mld}_{x}\left(Y, \mathfrak{a} \mathcal{O}_{Y}(-D)\right)=\operatorname{mld}_{x}\left(D, \mathfrak{a} \mathcal{O}_{D}\right) .
$$

By Theorem 1.4, Theorem 1.2 can be reduced to the case (1.1.4) when $X$ has quotient singularities. Hence this paper is mainly devoted to proving Theorem 1.4.

The main tools of this paper involve the theory of the arc space of a quotient singularity established by Denef and Loeser in [DL02] and the technique on arc spaces for proving (1.3.1) established by Ein, Mustaţă and Yasuda in [EMY03].

By the theory of Denef and Loeser, the arc space of quotient variety can be studied by those of certain $k[t]$-schemes. We briefly review their theory here. Suppose that a finite group $G \subset \mathrm{GL}_{N}(k)$ of order $d$ acts on $\bar{X}=\operatorname{Spec} k\left[x_{1}, \ldots, x_{N}\right]$. Let $\bar{Y} \subset \bar{X}$ be a $G$-invariant closed subvariety and $I \subset k\left[x_{1}, \ldots, x_{N}\right]$ its defining ideal. We denote by $Y:=\bar{Y} / G$ its quotient. For each $\gamma \in G$, a $k[t]$-scheme $\bar{Y}^{(\gamma)}$ is defined as follows. By changing the basis $x_{1}, \ldots, x_{N}$ linearly, we may assume that $\gamma$ is a diagonal matrix with entries $\xi^{e_{1}}, \ldots, \xi^{e_{N}}$ (for $0 \leqslant e_{i} \leqslant d-1$ ), where $\xi$ is a primitive $d$ th root of unity in $k$. Let $\bar{\lambda}_{\gamma}^{*}$ be the ring homomorphism defined by

$$
\bar{\lambda}_{\gamma}^{*}: k\left[x_{1}, \ldots, x_{N}\right]^{G} \rightarrow k[t]\left[x_{1}, \ldots, x_{N}\right], \quad x_{i} \mapsto t^{e_{i} / d} x_{i}
$$

Then the $k[t]$-scheme $\bar{Y}^{(\gamma)}$ is defined by

$$
\bar{Y}^{(\gamma)}=\operatorname{Spec} k[t]\left[x_{1}, \ldots, x_{N}\right] / \bar{I}^{(\gamma)},
$$




\section{Y. NAKAMURA AND K. ShiBATA}

where $\bar{I}^{(\gamma)}$ is the ideal generated by elements of $\bar{\lambda}_{\gamma}^{*}(I)$. We denote by $Y_{\infty}$ the arc space of $Y$, which parametrizes $k$-morphisms Spec $k[[t]] \rightarrow Y$. We also denote by $\bar{Y}_{\infty}^{(\gamma)}$ the arc space of $\bar{Y}^{(\gamma)}$ which parametrizes $k[t]$-morphisms Spec $k[[t]] \rightarrow \bar{Y}^{(\gamma)}$. In [DL02], Denef and Loeser investigate the change of variables formula on the map $\bar{Y}_{\infty}^{(\gamma)} \rightarrow Y_{\infty}$. Their theory allows us to compare the two spaces $Y_{\infty}$ and $\bigsqcup_{\gamma \in G} \bar{Y}_{\infty}^{(\gamma)}$. In [DL02], they basically work in the case where $I=0$ since they are interested in the quotient singularity. In Sections 2 and 3, we explain their theory in a more general setting in detail. We will give self-contained proofs of most of the propositions. However, we emphasize that they are not originally ours. Most of the propositions in Section 2 follow from existing works by Sebag [Seb04] and Yasuda [Yas19] (cf. [Yas04, Yas06]). In [Seb04], Sebag develops the theory of motivic integration for formal schemes over a complete discrete valuation ring. In [Yas19], Yasuda develops it for formal Deligne-Mumford stacks over $k[[t]]$ of arbitrary characteristic. The construction in Section 3 is a special case of the construction in [Yas16]. The construction in [Yas16] is intrinsic and more general, and it works even in positive characteristics. See Remarks 2.14 and 3.9 for more detail.

By the result of Ein, Mustaţă and Yasuda in [EMY03] (and [EM09]), the minimal log discrepancy of $Y$ can be described by the codimension of certain contact loci in $Y_{\infty}$. Then by applying the theory of Denef and Loeser above, it can be described by the codimension of the corresponding contact loci in $\bar{Y}_{\infty}^{(\gamma)}$. This description (Theorem 4.8) is one of the key steps to prove Theorem 1.4.

In Section 4, to prove Theorem 1.4, we apply the technique of Ein, Mustaţă and Yasuda in [EMY03] (and [EM09]) to the contact loci in $\bar{Y}_{\infty}^{(\gamma)}$. Their argument basically works well even in our setting because $\bar{I}^{(\gamma)}$ is generated by a regular sequence outside $t=0$ when $I$ is generated by a regular sequence. However, there are two main difficulties in this step, as we discuss below.

The first difficulty is that $\bar{Y}^{(\gamma)}$ is neither normal nor a complete intersection in general (see Remark 4.4). Therefore, we have no standard definition of the relative canonical sheaf $\omega_{\bar{Y}^{(\gamma)} / k[t]}$ on $\bar{Y}^{(\gamma)}$. We overcome this difficulty by defining an invertible sheaf $L_{\bar{Y}(\gamma)}$ instead. Furthermore, Lemma 4.7, which relates the age of $\gamma$ and certain orders of arcs, is a key lemma for the argument in [EMY03] to work in our setting.

The second difficulty is that there may be very few arcs on $\bar{Y}^{(\gamma)}$. More precisely, the arc space $\bar{Y}_{\infty}^{(\gamma)}$ may be a thin set of $\bar{Y}_{\infty}^{(\gamma)}$ itself (see Definition 2.21 for the definition of a thin set). For example, if $\left(d, e_{1}, e_{2}, e_{3}\right)=(3,0,1,2)$ and $I=\left(x_{1}^{3}+x_{2}^{3}+x_{3}^{3}\right)$, then we have

$$
\bar{Y}^{(\gamma)}=\operatorname{Spec} k[t]\left[x_{1}, x_{2}, x_{3}\right] /\left(x_{1}^{3}+t x_{2}^{3}+t^{2} x_{3}^{3}\right) .
$$

In this case, $\bar{Y}_{\infty}^{(\gamma)}$ consists of only one arc, and the order of the Jacobian ideal $\operatorname{Jac}_{\bar{Y}^{(\gamma)} / k[t]}$ is infinity. If the arc space $\bar{Y}_{\infty}^{(\gamma)}$ is a thin set, then any arc $\alpha \in \bar{Y}_{\infty}^{(\gamma)}$ has order $\operatorname{ord}_{\alpha}\left(\operatorname{Jac}_{\bar{Y}^{(\gamma)} / k[t]}\right)=\infty$, and because of this, the argument in [EMY03] does not work. In Claim 5.2, we prove that $\bar{Y}_{\infty}^{(\gamma)}$ is not a thin set if $Y$ is klt and show that the argument in [EMY03] really works. The key idea in the proof of Claim 5.2 is to apply the result by Hacon and McKernan [HM07], which states the rational chain connectedness of the fibers of the resolution $W \rightarrow \bar{Y}^{(\gamma)}$ of singularities of $\bar{Y}^{(\gamma)}$, and prove that there are actually many arcs on $W$ using the result by Graber, Harris and Starr [GHS03]. The klt assumption in Theorem 1.4 is essentially used in this argument. 


\section{INVERSION OF ADJUNCTION FOR QUOTIENT SINGULARITIES}

We also prove the Reid-Tai type formula on minimal log discrepancies (Corollary 4.12). This gives the affirmative answer to a question by Borisov in [Bor97] of whether the set of minimal discrepancies of quotient singularities with respect to arbitrary groups coincides with that of cyclic quotients of the same dimension. We prove Corollary 4.12 using the description of the minimal log discrepancy in terms of arc spaces of the $k[t]$-schemes. Moreover, we give another proof without the theory of arc spaces. As an application of this result, we prove the ACC conjecture for quotient singularities (Theorem 4.14).

The paper is organized as follows. In Section 2, we review some definitions and facts on pairs and arc spaces. We also prove some basic results on the arc spaces of $k[t]$-schemes, which are necessary for this paper. In particular, we discuss the theory of contact loci and their codimension for the arc spaces of $k[t]$-schemes following [EM09], where the arc spaces of $k$-schemes are dealt with. In Section 3, we review the theory of arc spaces of quotient varieties established by Denef and Loeser in [DL02]. As mentioned previously, most of the propositions in Sections 2 and 3 follow from existing works by Sebag [Seb04] and Yasuda [Yas19, Yas16]. Readers who are familiar with these papers could skip these sections. In Section 4, we discuss the minimal log discrepancy of quotient singularities of linear action and describe them by the codimension of cylinders in arc spaces of the $k[t]$-schemes defined in the previous section (Theorem 4.8). In Section 5 , we prove the PIA conjecture for hyperquotient singularities (Theorem 5.1). In Section 6, we prove the main theorems Corollary 6.1 and Theorem 6.2 with some generalizations.

\section{Preliminaries}

\subsection{Notation}

- We basically follow the notation and the terminology in [Har77] and [Kol13].

- Throughout this paper, $k$ is an algebraically closed field of characteristic zero. We say that $X$ is a variety over $k$ or a $k$-variety if $X$ is an integral scheme that is separated and of finite type over $k$.

2.2. Log pairs. A log pair $(X, \mathfrak{a})$ is a normal $\mathbb{Q}$-Gorenstein variety $X$ and an $\mathbb{R}$-ideal sheaf $\mathfrak{a}$ on $X$. Here, an $\mathbb{R}$-ideal sheaf $\mathfrak{a}$ on $X$ is a formal product $\mathfrak{a}=\prod_{i=1}^{s} \mathfrak{a}_{i}^{r_{i}}$, where $\mathfrak{a}_{1}, \ldots, \mathfrak{a}_{s}$ are non-zero coherent ideal sheaves on $X$ and $r_{1}, \ldots, r_{s}$ are positive real numbers. For a morphism $Y \rightarrow X$ and an $\mathbb{R}$-ideal sheaf $\mathfrak{a}=\prod_{i=1}^{s} \mathfrak{a}_{i}^{r_{i}}$, we denote by $\mathfrak{a} \mathcal{O}_{Y}$ the $\mathbb{R}$-ideal sheaf $\prod_{i=1}^{s}\left(\mathfrak{a}_{i} \mathcal{O}_{Y}\right)^{r_{i}}$ on $Y$.

Let $\left(X, \mathfrak{a}=\prod_{i=1}^{s} \mathfrak{a}_{i}^{r_{i}}\right)$ be a log pair. For a proper birational morphism $f: X^{\prime} \rightarrow X$ from a normal variety $X^{\prime}$ and a prime divisor $E$ on $X^{\prime}$, the log discrepancy of $(X, \mathfrak{a})$ at $E$ is defined as

$$
a_{E}(X, \mathfrak{a}):=1+\operatorname{ord}_{E}\left(K_{X^{\prime}}-f^{*} K_{X}\right)-\operatorname{ord}_{E}(\mathfrak{a}),
$$

where we set $\operatorname{ord}_{E}(\mathfrak{a})=\sum_{i=1}^{s} r_{i} \operatorname{ord}_{E}\left(\mathfrak{a}_{i}\right)$. The image $f(E)$ is called the center of $E$ on $X$; we denote it by $c_{X}(E)$. For a closed point $x \in X$, we define the minimal log discrepancy at $x$ as

$$
\operatorname{mld}_{x}(X, \mathfrak{a}):=\inf _{c_{X}(E)=\{x\}} a_{E}(X, \mathfrak{a})
$$

if $\operatorname{dim} X \geqslant 2$, where the infimum is taken over all prime divisors $E$ over $X$ with center $c_{X}(E)=\{x\}$. It is known that $\operatorname{mld}_{x}(X, \mathfrak{a}) \in \mathbb{R}_{\geqslant 0} \cup\{-\infty\}$ in this case (cf. [KM98, Corollary 2.31]). When $\operatorname{dim} X=1$, we define $\operatorname{mld}_{x}(X, \mathfrak{a}):=\inf _{c_{X}(E)=\{x\}} a_{E}(X, \mathfrak{a})$ if the infimum is non-negative and $\operatorname{mld}_{x}(X, \mathfrak{a}):=-\infty$ otherwise. 


\section{Y. NAKAMURA AND K. ShiBATA}

2.3. Jet schemes and arc spaces for $\boldsymbol{k}$-schemes. In this subsection, we briefly review the definition and some properties of jet schemes and arc spaces. The reader is referred to [EM09] for details.

Let $X$ be a scheme of finite type over $k$, and let (Sch/ $k$ ) be the category of $k$-schemes and (Sets) the category of sets. Define a contravariant functor $F_{m}:(\operatorname{Sch} / k) \rightarrow($ Sets) by

$$
F_{m}(Y)=\operatorname{Hom}_{k}\left(Y \times \operatorname{Spec} k \operatorname{Spec} k[t] /\left(t^{m+1}\right), X\right) .
$$

Then, the functor $F_{m}$ is representable by a scheme $X_{m}$ of finite type over $k$, and the scheme $X_{m}$ is called the $m$ th jet scheme of $X$. For $m \geqslant n \geqslant 0$, the canonical surjective homomorphism $k[t] /\left(t^{m+1}\right) \rightarrow k[t] /\left(t^{n+1}\right)$ induces a morphism $\pi_{m n}: X_{m} \rightarrow X_{n}$. We have the projective limit and projections

$$
X_{\infty}:=\underbrace{\lim }_{m} X_{m}, \quad \psi_{m}: \quad X_{\infty} \rightarrow X_{m},
$$

and $X_{\infty}$ is called the arc space of $X$. There is a bijective map

$$
\operatorname{Hom}_{k}\left(\operatorname{Spec} K, X_{\infty}\right) \simeq \operatorname{Hom}_{k}(\operatorname{Spec} K[[t]], X)
$$

for any field $K$ with $k \subset K$.

For $m \in \mathbb{Z}_{\geqslant 0} \cup\{\infty\}$, we denote by $\pi_{m}: X_{m} \rightarrow X$ the canonical truncation morphism. For $m \in \mathbb{Z}_{\geqslant 0} \cup\{\infty\}$ and a morphism $f: Y \rightarrow X$ of schemes of finite type over $k$, we denote by $f_{m}: Y_{m} \rightarrow X_{m}$ the morphism induced by $f$.

A subset $C \subset X_{\infty}$ is called a cylinder if $C=\psi_{m}^{-1}(S)$ holds for some $m \geqslant 0$ and a constructible subset $S \subset X_{m}$. Typical examples of cylinders appearing in this paper are the contact loci Cont $^{m}(\mathfrak{a})$ and Cont ${ }^{\geqslant m}(\mathfrak{a})$ defined as follows.

Definition 2.1. (1) For an arc $\gamma \in X_{\infty}$ and an ideal sheaf $\mathfrak{a} \subset \mathcal{O}_{X}$, the order of $\mathfrak{a}$ measured by $\gamma$ is defined as

$$
\operatorname{ord}_{\gamma}(\mathfrak{a})=\sup \left\{r \in \mathbb{Z}_{\geqslant 0} \mid \gamma^{*}(\mathfrak{a}) \subset\left(t^{r}\right)\right\},
$$

where $\gamma^{*}: \mathcal{O}_{X} \rightarrow k[[t]]$ is the ring homomorphism induced by $\gamma$.

(2) For $m \in \mathbb{Z}_{\geqslant 0}$, we define $\operatorname{Cont}^{m}(\mathfrak{a})$, Cont ${ }^{\geqslant m}(\mathfrak{a}) \subset X_{\infty}$ as

$$
\begin{aligned}
\operatorname{Cont}^{m}(\mathfrak{a}) & =\left\{\gamma \in X_{\infty} \mid \operatorname{ord}_{\gamma}(\mathfrak{a})=m\right\}, \\
\operatorname{Cont}^{\geqslant m}(\mathfrak{a}) & =\left\{\gamma \in X_{\infty} \mid \operatorname{ord}_{\gamma}(\mathfrak{a}) \geqslant m\right\} .
\end{aligned}
$$

By definition, we can see that

$$
\text { Cont }^{\geqslant m}(\mathfrak{a})=\psi_{m-1}^{-1}\left(Z(\mathfrak{a})_{m-1}\right),
$$

where $Z(\mathfrak{a})$ is the closed subscheme of $X$ defined by the ideal sheaf $\mathfrak{a}$. Therefore, $\operatorname{Cont}^{m}(\mathfrak{a})$ and Cont $\geqslant m(\mathfrak{a})$ become cylinders.

For $m \leqslant n+1$, we also define the subsets $\operatorname{Cont}^{m}(\mathfrak{a})_{n}$ and Cont ${ }^{\geqslant m}(\mathfrak{a})_{n}$ of $X_{n}$ in the same way.

We shall define the codimension for cylinders. For a variety $X$ of dimension $n$, we denote by $\operatorname{Jac}_{X}:=\operatorname{Fitt}^{n}\left(\Omega_{X}\right)$ the Jacobian ideal of $X$ and by $X_{\text {sing }}$ the singular locus of $X$ (see [Eis95] for the definition of the Fitting ideal).

Definition 2.2. Let $X$ be a variety, and let $C \subset X_{\infty}$ be a cylinder.

(1) Assume that $C \subset \operatorname{Cont}^{e}\left(\operatorname{Jac}_{X}\right)$ for some $e \in \mathbb{Z}_{\geqslant 0}$. Then we define the codimension of $C$ in $X_{\infty}$ as

$$
\operatorname{codim}(C):=(m+1) \operatorname{dim} X-\operatorname{dim}\left(\psi_{m}(C)\right)
$$




\section{INVERSION OF ADJUNCTION FOR QUOTIENT SINGULARITIES}

for any sufficiently large $m$. This is well defined by [EM09, Proposition 4.1].

(2) In general, we define the codimension of $C$ in $X_{\infty}$ as

$$
\operatorname{codim}(C):=\min _{e \in \mathbb{Z}_{\geqslant 0}} \operatorname{codim}\left(C \cap \operatorname{Cont}^{e}\left(\operatorname{Jac}_{X}\right)\right) .
$$

By convention, $\operatorname{codim}(C)=\infty$ if $C \subset\left(X_{\text {sing }}\right)_{\infty}$.

We recall the definition of the Nash ideals of varieties and morphisms.

Definition 2.3. (1) Let $X$ be a normal $\mathbb{Q}$-Gorenstein variety over $k$ of dimension $n$, and let $r$ be a positive integer such that the reflexive power $\omega_{X}^{[r]}:=\left(\omega_{X}^{\otimes r}\right)^{* *}$ is an invertible sheaf. Then we have a canonical map

$$
\eta_{r}:\left(\Omega_{X}^{n}\right)^{\otimes r} \rightarrow \omega_{X}^{[r]} .
$$

Since $\omega_{X}^{[r]}$ is an invertible sheaf, an ideal sheaf $\mathfrak{n}_{r, X} \subset \mathcal{O}_{X}$ is uniquely determined by $\operatorname{Im}\left(\eta_{r}\right)=$ $\mathfrak{n}_{r, X} \otimes \omega_{X}^{[r]}$. The ideal sheaf $\mathfrak{n}_{r, X}$ is called the $r$ th Nash ideal of $X$.

(2) Furthermore, let $f: X \rightarrow Y$ be a morphism to a variety $Y$ over $k$. Then we have a canonical map

$$
\theta_{r}: f^{*}\left(\Omega_{Y}^{n}\right)^{\otimes r} \rightarrow \omega_{X}^{[r]}
$$

and an ideal sheaf $\mathfrak{n}_{r, f} \subset \mathcal{O}_{X}$ such that $\operatorname{Im}\left(\theta_{r}\right)=\mathfrak{n}_{r, f} \otimes \omega_{X}^{[r]}$. The ideal sheaf $\mathfrak{n}_{r, f}$ is called the $r$ th Nash ideal of $f$.

2.4. Jet schemes and arc spaces for $\boldsymbol{k}[\boldsymbol{t}]$-schemes. Following [DL02], we extend the definition of the arc spaces of $k$-schemes in Subsection 2.3 to the case where $X$ is a $k[t]$-scheme, namely a scheme over $\operatorname{Spec} k[t]$.

Let $X$ be a scheme of finite type over Spec $k[t]$. Define a contravariant functor $F_{m}:(\mathrm{Sch} / k) \rightarrow$ (Sets) by

$$
F_{m}(Y)=\operatorname{Hom}_{k[t]}\left(Y \times_{\text {Spec } k} \operatorname{Spec} k[t] /\left(t^{m+1}\right), X\right) .
$$

Then, $F_{m}$ is representable by a scheme $X_{m}$ of finite type over $k$, and the scheme $X_{m}$ is called the $m$ th jet scheme of $X$. We shall also use the same symbols $X_{\infty}, \pi_{m n}, \psi_{m}, \pi_{m}$ for this setting. Cylinders and the contact loci $\operatorname{Cont}^{m}(\mathfrak{a})$ and $\operatorname{Cont}^{\geqslant m}(\mathfrak{a})$ are also defined in the same way for this setting.

Remark 2.4. Note that $X_{0} \simeq X$ holds if $X$ is a scheme over $k$. However, this is not true for $k[t]$-schemes. Indeed, if $X=\mathbb{A}_{k[t]}^{1}$, then $X_{0} \simeq \mathbb{A}_{k}^{1}$ holds. More generally, $X_{m} \simeq Y_{m}$ holds for a $k$-scheme $X$ and $Y=X \times_{\text {Spec } k} \operatorname{Spec} k[t]$.

In this paper, we basically treat $k[t]$-schemes with one of the following two conditions:

$(\star)_{n}$ The scheme $X$ is of finite type over Spec $k[t]$. Any irreducible component of $X$ has dimension at least $n+1$. Furthermore, any irreducible component dominating Spec $k[t]$ is exactly $(n+1)$-dimensional.

$(\star \star)_{n}$ The scheme $X$ is a $k[t]$-scheme with the condition $(\star)_{n}$. Furthermore, any irreducible components of $X$ dominating Spec $k[t]$ are reduced outside $t=0$.

These categories are suitable for defining the Jacobian ideal and the codimension of cylinders.

For a $k[t]$-schemes $X$ with the condition $(\star)_{n}$, we denote by $\operatorname{Jac}_{X / k[t]}:=\operatorname{Fitt}^{n}\left(\Omega_{X / k[t]}\right)$ the Jacobian ideal of $X$ over $k[t]$. Under the condition $(\star \star)_{n}$, we will see in Subsection 2.5 that the codimension of a cylinder is also defined in the same way as in Definition 2.2. 


\section{Y. NAKAMURA AND K. ShiBATA}

Remark 2.5. Let $X$ be a $k[t]$-scheme with the condition $(\star)_{n}$. Let $X^{(1)}, \ldots, X^{(\ell)}$ be the irreducible components of $X$ dominating Spec $k[t]$. Then we have $X_{\infty}=\bigcup_{i=1}^{\ell} X_{\infty}^{(i)}$. Therefore, we can reduce some problems on the arc space of $X$ to problems for its irreducible component dominating Spec $k[t]$. However, we cannot do such a reduction on problems relating to the order of the Jacobian ideal (cf. Example 2.6).

Example 2.6. The $k[t]$-schemes

$$
X=\operatorname{Spec}(k[t][x, y, z] /(t x, t y)), \quad Y=\operatorname{Spec}(k[t][x, y, z] /(x, y))
$$

satisfy the condition $(\star \star)_{1}$. We have a canonical isomorphism $X_{\infty} \simeq Y_{\infty}$ on the arc spaces, but corresponding arcs have different orders of the Jacobian ideals because

$$
\operatorname{Jac}_{X / k[t]}=\left(t^{2}, t x, t y\right) /(t x, t y) \quad \text { and } \quad \operatorname{Jac}_{Y / k[t]}=(1) .
$$

We also define the order of the Jacobian for a morphism.

Definition 2.7. Let $X$ and $Y$ be $k[t]$-schemes of finite type, and let $f: X \rightarrow Y$ be a morphism over $k[t]$. Let $\gamma$ : Spec $k[[t]] \rightarrow X$ be an arc, and let $\gamma^{\prime}:=f_{\infty}(\gamma)$. Let $S$ be the torsion part of $\gamma^{*} \Omega_{X / k[t]}$. Then we define the order $\operatorname{ord}_{\gamma}\left(\mathrm{jac}_{f}\right)$ of the Jacobian of $f$ at $\gamma$ as the length of the $k[[t]]$-module

$$
\operatorname{Coker}\left(\gamma^{\prime *} \Omega_{Y / k[t]} \rightarrow \gamma^{*} \Omega_{X / k[t]} / S\right)
$$

In particular, if $\operatorname{ord}_{\gamma}\left(\mathrm{jac}_{f}\right)<\infty$, then

$$
\operatorname{Coker}\left(\gamma^{\prime *} \Omega_{Y / k[t]} \rightarrow \gamma^{*} \Omega_{X / k[t]} / S\right) \simeq \bigoplus_{i} k[t] /\left(t^{e_{i}}\right)
$$

holds as $k[[t]]$-modules with some positive integers $e_{i}$ satisfying $\sum_{i} e_{i}=\operatorname{ord}_{\gamma}\left(\mathrm{jac}_{f}\right)$.

By abuse of notation, we set

$$
\operatorname{Cont}^{e}\left(\mathrm{jac}_{f}\right):=\left\{\gamma \in X_{\infty} \mid \operatorname{ord}_{\gamma}\left(\mathrm{jac}_{f}\right)=e\right\}
$$

for $e \in \mathbb{Z}_{\geqslant 0}$. We note that it is not clear from the definition that $\operatorname{Cont}^{e}\left(\mathrm{jac}_{f}\right)$ is a cylinder.

Remark 2.8. In some papers, the Jacobian ideal $\operatorname{Jac}_{f}$ of $f$ is defined by $\operatorname{Jac}_{f}:=\operatorname{Fitt}^{0}\left(\Omega_{X / Y}\right)$. We note that $\operatorname{ord}_{\gamma}\left(\operatorname{Jac}_{f}\right)$ coincides with the length of the $k[[t]]$-module

$$
\operatorname{Coker}\left(\gamma^{\prime *} \Omega_{Y / k[t]} \rightarrow \gamma^{*} \Omega_{X / k[t]}\right)=\gamma^{*} \Omega_{X / Y}
$$

Therefore, if $\Omega_{X / k[t]}$ is locally free, then $\operatorname{ord}_{\gamma}\left(\operatorname{Jac}_{f}\right)=\operatorname{ord}_{\gamma}\left(\operatorname{jac}_{f}\right)$ holds. However, the equality does not hold in general (cf. Example 2.9).

Example 2.9. Let $R=k[t][x, y, z] /\left(x y+z^{2}\right)$, and let $f: R \rightarrow R$ be the homomorphism defined by $f(x)=x^{2}, f(y)=x y$ and $f(z)=x z$. Let $\gamma: R \rightarrow k[[t]]$ be the arc defined by $\gamma(x)=t$ and $\gamma(y)=\gamma(z)=0$. Then $\gamma^{*} \Omega_{R / k[t]}=k[[t]] d x \oplus(k[[t]] /(t)) d y \oplus k[[t]] d z$. Note that $d(f(x))=$ $2 x d x, d(f(y))=y d x+x d y$ and $d(f(z))=z d x+x d z$. Therefore, we have $\operatorname{ord}_{\gamma}\left(\operatorname{Jac}_{f}\right)=3$ and $\operatorname{ord}_{\gamma}\left(\mathrm{jac}_{f}\right)=2$.

Additivity holds for the orders of the Jacobian of morphisms.

Lemma 2.10. Let $n$ be a non-negative integer, and let $X, Y$ and $Z$ be $k[t]$-schemes with the condition $(\star)_{n}$. Let $f: X \rightarrow Y$ and $g: Y \rightarrow Z$ be morphisms over $k[t]$. Let $\gamma \in X_{\infty}$ be an arc, and let $\gamma^{\prime}:=f_{\infty}(\gamma)$. Suppose that

$$
\operatorname{ord}_{\gamma}\left(\operatorname{Jac}_{X / k[t]}\right)<\infty, \quad \operatorname{ord}_{\gamma^{\prime}}\left(\operatorname{Jac}_{Y / k[t]}\right)<\infty .
$$


Then we have

$$
\operatorname{ord}_{\gamma}\left(\operatorname{jac}_{g \circ f}\right)=\operatorname{ord}_{\gamma}\left(\operatorname{jac}_{f}\right)+\operatorname{ord}_{\gamma^{\prime}}\left(\operatorname{jac}_{g}\right) .
$$

Proof. Set $\gamma^{\prime \prime}:=g_{\infty}\left(\gamma^{\prime}\right)$. Let $S, T$ and $U$ be the torsion parts of $\gamma^{*} \Omega_{X / k[t]}, \gamma^{\prime *} \Omega_{Y / k[t]}$ and $\gamma^{\prime \prime *} \Omega_{Z / k[t]}$, respectively. Since $\operatorname{ord}_{\gamma}\left(\operatorname{Jac}_{X / k[t]}\right)<\infty$, the scheme $X$ is smooth over $k[t]$ at $\gamma(\eta)$, where $\eta$ is the generic point of Spec $k[[t]]$. Hence we have $\gamma^{*} \Omega_{X / k[t]} / S \simeq k[[t]]{ }^{\oplus n}$. For the same reason, we have $\gamma^{\prime *} \Omega_{Y / k[t]} / T \simeq k[[t]]^{\oplus n}$. If

$$
\operatorname{ord}_{\gamma}\left(\operatorname{jac}_{f}\right)=\operatorname{length}\left(\operatorname{Coker}\left(\gamma^{\prime *} \Omega_{Y / k[t]} / T \rightarrow \gamma^{*} \Omega_{X / k[t]} / S\right)\right)=\infty
$$

holds, then we have

$$
\operatorname{ord}_{\gamma}\left(\operatorname{jac}_{g \circ f}\right)=\operatorname{length}\left(\operatorname{Coker}\left(\gamma^{\prime \prime *} \Omega_{Z / k[t]} / U \rightarrow \gamma^{*} \Omega_{X / k[t]} / S\right)\right)=\infty .
$$

Otherwise, $\gamma^{\prime *} \Omega_{Y / k[t]} / T \rightarrow \gamma^{*} \Omega_{X / k[t]} / S$ is injective, and the additivity $\operatorname{ord}_{\gamma}\left(\mathrm{jac}_{g \circ f}\right)=\operatorname{ord}_{\gamma}\left(\mathrm{jac}_{f}\right)$ $+\operatorname{ord}_{\gamma^{\prime}}\left(\mathrm{jac}_{g}\right)$ follows from the additivity of the length of modules.

Nash ideals can also be defined in this setting.

Definition 2.11. Let $X$ be a normal $k[t]$-variety of relative dimension $n$. Suppose that $X$ is smooth over $k[t]$ outside a closed subset of $X$ of codimension two. Then the canonical sheaf $\omega_{X / k[t]}$ is defined (cf. [Kol13, Definition 1.6]). Suppose that there exists a positive integer $r$ such that $\omega_{X / k[t]}^{[r]}$ is an invertible sheaf.

(1) Then we have a canonical map

$$
\eta_{r}:\left(\Omega_{X / k[t]}^{n}\right)^{\otimes r} \rightarrow \omega_{X / k[t]}^{[r]} .
$$

Since $\omega_{X / k[t]}^{[r]}$ is an invertible sheaf, an ideal sheaf $\mathfrak{n}_{r, X} \subset \mathcal{O}_{X}$ is uniquely determined by $\operatorname{Im}\left(\eta_{r}\right)=\mathfrak{n}_{r, X} \otimes \omega_{X / k[t]}^{[r]}$. The ideal sheaf $\mathfrak{n}_{r, X}$ is called the $r$ th Nash ideal of $X$.

(2) Furthermore, let $f: X \rightarrow Y$ be a $k[t]$-morphism from a $k[t]$-scheme $Y$. Then we have a canonical map

$$
\theta_{r}: f^{*}\left(\Omega_{Y / k[t]}^{n}\right)^{\otimes r} \rightarrow \omega_{X / k[t]}^{[r]}
$$

and an ideal sheaf $\mathfrak{n}_{r, f} \subset \mathcal{O}_{X}$ such that $\operatorname{Im}\left(\theta_{r}\right)=\mathfrak{n}_{r, f} \otimes \omega_{X / k[t]}^{[r]}$. The ideal sheaf $\mathfrak{n}_{r, f}$ is called the $r$ th Nash ideal of $f$.

Remark 2.12. In this paper, we only use this definition for a $k[t]$-variety $X^{\prime}$ of the form $X^{\prime}=$ $X \times_{\text {Spec } k}$ Spec $k[t]$, where $X$ is a normal $k$-variety. In this case, $\omega_{X^{\prime} / k[t]}$ is just the pull-back of $\omega_{X}$ to $X^{\prime}$. Therefore, $\mathfrak{n}_{r, X^{\prime}}=\mathfrak{n}_{r, X} \mathcal{O}_{X^{\prime}}$ holds.

Lemma 2.13. (1) Let $X$ be a $k[t]$-scheme with the condition $(\star)_{n}$, and let $\gamma \in \operatorname{Cont}^{e}\left(\mathrm{Jac}_{X / k[t]}\right)$ be an arc. Then

$$
\gamma^{*} \Omega_{X / k[t]} \simeq k[[t]]^{\oplus n} \oplus \bigoplus_{i} k[t] /\left(t^{e_{i}}\right)
$$

holds as $k[[t]]$-modules with $\sum_{i} e_{i}=e$.

(2) Let $r$ be a positive integer and $f: X \rightarrow Y$ be a $k[t]$-morphism which satisfy the assumption of Definition 2.11(2). Let $\gamma \in X_{\infty}$ be an arc. Suppose $\operatorname{ord}_{\gamma}\left(\operatorname{Jac}_{X / k[t]}\right)<\infty$. Then

$$
r \operatorname{ord}_{\gamma}\left(\operatorname{jac}_{f}\right)+\operatorname{ord}_{\gamma}\left(\mathfrak{n}_{r, X}\right)=\operatorname{ord}_{\gamma}\left(\mathfrak{n}_{r, f}\right)
$$

holds. 


\section{Y. NAKAMURA AND K. ShiBATA}

Proof. First we prove assertion (1). Since $k[[t]]$ is a principal ideal domain, the finitely generated module $\gamma^{*} \Omega_{X / k[t]}$ is isomorphic to a module of the form $k[[t]]^{\oplus a} \oplus \bigoplus_{i} k[t] /\left(t^{e_{i}}\right)$. Since $\operatorname{ord}_{\gamma}\left(\operatorname{Jac}_{X / k[t]}\right)=e<\infty$, the scheme $X$ is smooth over $k[t]$ at $\gamma(\eta)$, where $\eta$ is the generic point of Spec $k[[t]]$. Therefore, we have $a=n$. Then the assertion follows from the definition of $\mathrm{Jac}_{X / k[t]}$ and

$$
\left(t^{\sum_{i} e_{i}}\right)=\operatorname{Fitt}^{n}\left(\gamma^{*} \Omega_{X / k[t]}\right)=\gamma^{*} \operatorname{Fitt}^{n}\left(\Omega_{X / k[t]}\right)=\gamma^{*} \operatorname{Jac}_{X / k[t]} .
$$

We now prove assertion (2). Let $S$ be the torsion part of $\gamma^{*} \Omega_{X / k[t]}$. Let $S^{\prime}$ be the torsion part of $\left(\gamma^{*} \Omega_{X / k[t]}^{n}\right)^{\otimes r}$. Since $\gamma^{*} \omega_{X / k[t]}^{[r]}$ is torsion-free, the map

$$
\eta_{r}:\left(\gamma^{*} \Omega_{X / k[t]}^{n}\right)^{\otimes r} \rightarrow \gamma^{*} \omega_{X / k[t]}^{[r]}
$$

factors through $\left(\gamma^{*} \Omega_{X / k[t]}^{n}\right)^{\otimes r} / S^{\prime}$. Hence we have

$$
\operatorname{ord}_{\gamma}\left(\mathfrak{n}_{r, X}\right)=\operatorname{length}\left(\operatorname{Coker} \eta_{r}\right)=\operatorname{length}\left(\operatorname{Coker}\left(\left(\gamma^{*} \Omega_{X / k[t]}^{n}\right)^{\otimes r} / S^{\prime} \rightarrow \gamma^{*} \omega_{X / k[t]}^{[r]}\right)\right) \text {. }
$$

In the same way, we have

$$
\operatorname{ord}_{\gamma}\left(\mathfrak{n}_{r, f}\right)=\operatorname{length}\left(\operatorname{Coker}\left(\left(\gamma^{\prime *} \Omega_{Y / k[t]}^{n}\right)^{\otimes r} / T^{\prime} \rightarrow \gamma^{*} \omega_{X / k[t]}^{[r]}\right)\right),
$$

where $\gamma^{\prime}=f_{\infty}(\gamma)$ and $T^{\prime}$ is the torsion part of $\left(\gamma^{\prime *} \Omega_{Y / k[t]}^{n}\right)^{\otimes r}$.

We note that

and we have

$$
\left(\gamma^{*} \Omega_{X / k[t]}^{n}\right)^{\otimes r} / S^{\prime} \simeq\left(\left(\gamma^{*} \Omega_{X / k[t]} / S\right)^{\wedge n}\right)^{\otimes r} \simeq k[[t]]
$$

$$
r \operatorname{ord}_{\gamma}\left(\operatorname{jac}_{f}\right)=\operatorname{length}\left(\operatorname{Coker}\left(\left(\gamma^{\prime *} \Omega_{Y / k[t]}^{n}\right){ }^{\otimes r} / T^{\prime} \rightarrow\left(\gamma^{*} \Omega_{X / k[t]}^{n}\right)^{\otimes r} / S^{\prime}\right)\right)
$$

by Definition 2.7. Then the desired formula

$$
r \operatorname{ord}_{\gamma}\left(\operatorname{jac}_{f}\right)+\operatorname{ord}_{\gamma}\left(\mathfrak{n}_{r, X}\right)=\operatorname{ord}_{\gamma}\left(\mathfrak{n}_{r, f}\right)
$$

follows from the additivity of the length of modules.

Remark 2.14. (1) In [Seb04], Sebag extends the theory of motivic integration for $k[t]$-schemes to the case of formal schemes over $k[[t]]$ with $k$ a perfect field. We also refer the reader to [CNS18] to this theory.

With a scheme $X$ of finite type over $k[t]$, we can associate the formal scheme $\mathcal{X}$ over $k[[t]]$ by

$$
\mathcal{X}:=\underset{i \geqslant 0}{\lim _{i}} \mathcal{X}_{i}, \quad \text { where } \mathcal{X}_{i}:=X \times_{\operatorname{Spec} k[t]} \operatorname{Spec}\left(k[[t]] /\left(t^{i+1}\right)\right) .
$$

Then the Greenberg schemes $\operatorname{Gr}_{m}(\mathcal{X})$ and $\operatorname{Gr}(\mathcal{X})$ defined in [Seb04] are isomorphic to $X_{m}$ and $X_{\infty}$, respectively (cf. [CNS18, Chapter 4, Example 3.3.3]). Therefore, the theory of the Greenberg schemes developed in [Seb04] and [CNS18] can be applied to the arc spaces $X_{\infty}$ of $k[t]$-schemes $X$.

When $\mathcal{X}$ is a formal scheme of finite type of relative dimension $d$ over $k[[t]]$, the Jacobian ideal $\operatorname{Jac}_{\mathcal{X}}$ is defined by $\operatorname{Jac}_{\mathcal{X}}=\operatorname{Fitt}^{d}\left(\Omega_{\mathcal{X} / k[t]]}\right)$ (see [CNS18, Chapter 5, Definition 1.3.1]). When $\mathcal{X}$ is the formal scheme associated with a scheme $X$ of finite type of relative dimension $d$ over $k[t]$, this definition is compatible with the definition of $\mathrm{Jac}_{X / k[t]}$ in the sense that

$$
\operatorname{Jac}_{\mathcal{X}} \mathcal{O}_{\mathcal{X}_{i}}=\operatorname{Jac}_{X / k[t]} \mathcal{O}_{\mathcal{X}_{i}}
$$

for each $i \geqslant 0$. This follows from the base change properties of the sheaves of differentials (cf. [Liu02, Chapter 6, Proposition 1.8(a)]) and the Fitting ideal (cf. [Eis95, Corollary 20.5]). For a morphism $h: \mathcal{X} \rightarrow \mathcal{Y}$ of formal schemes of finite type over $k[[t]]$ and for $\gamma \in \operatorname{Gr}(\mathcal{X})$, the order 
$\operatorname{ord}_{t}(\mathrm{Jac})_{h}(\gamma)$ is defined in [Seb04, Section 5]; it is denoted by $\operatorname{ordjac}_{h}(\gamma)$ in [CNS18, Chapter 5, Section 3.1.1]. This corresponds to $\operatorname{ord}_{\gamma}\left(\mathrm{jac}_{h}\right)$ in Definition 2.7 when $h: \mathcal{X} \rightarrow \mathcal{Y}$ is induced by a $k[t]$-morphism $h: X \rightarrow Y$ of $k[t]$-schemes.

(2) Theorems in [Seb04] and [CNS18] often assume that the formal schemes are flat over $k[[t]]$. However, many of them can be applied to non-flat formal schemes as well. In particular, they can be applied to $k[t]$-schemes with the condition $(\star)_{n}$, as we will see in Subsection 2.5.

(3) Let $X$ be a $k[t]$-scheme with the condition $(\star)_{n}$. Let $X^{\prime}$ be the maximal closed subscheme of $X$ which is flat over Spec $k[t]$. Then we have $X_{\infty}^{\prime}=X_{\infty}$, though the inclusion $X_{m}^{\prime} \subset X_{m}$ is not necessarily an equality. Let $I_{X^{\prime}} \subset \mathcal{O}_{X}$ be the defining ideal of $X^{\prime}$. Then there exists a non-negative integer $a$ such that $t^{a} I_{X^{\prime}}=0$ holds in a neighborhood of $t=0$. It follows that $\pi_{m+a, m}\left(X_{m+a}\right) \subset X_{m}^{\prime}$.

Furthermore, $\operatorname{ord}_{\gamma}\left(\mathrm{Jac}_{X^{\prime} / k[t]}\right) \neq \operatorname{ord}_{\gamma}\left(\operatorname{Jac}_{X / k[t]}\right)$ holds for $\gamma \in X_{\infty}$ in general (Example 2.6). However, as we will see below, the difference is bounded. Since $t^{a} I_{X^{\prime}}=0$ holds, there exists a non-negative integer $a^{\prime}$ such that

$$
t^{a^{\prime}} p^{-1}\left(\operatorname{Jac}_{X^{\prime} / k[t]}\right) \subset \operatorname{Jac}_{X / k[t]}
$$

in a neighborhood of $t=0$, where we set $p: \mathcal{O}_{X} \rightarrow \mathcal{O}_{X^{\prime}}$. Hence we have

$$
\operatorname{ord}_{\gamma}\left(\operatorname{Jac}_{X^{\prime} / k[t]}\right) \leqslant \operatorname{ord}_{\gamma}\left(\operatorname{Jac}_{X / k[t]}\right) \leqslant a^{\prime}+\operatorname{ord}_{\gamma}\left(\operatorname{Jac}_{X^{\prime} / k[t]}\right)
$$

for any $\gamma \in X_{\infty}$.

Let $h: X \rightarrow Y$ be a $k[t]$-morphism of $k[t]$-schemes with the condition $(\star)_{n}$. Let $Y^{\prime}$ be the maximal closed subscheme of $Y$ which is flat over Spec $k[t]$. Then $h$ induces a $k[t]$-morphism $h^{\prime}: X^{\prime} \rightarrow Y^{\prime}$. It follows that $\operatorname{ord}_{\gamma}\left(\operatorname{jac}_{h}\right)=\operatorname{ord}_{\gamma}\left(\operatorname{jac}_{h^{\prime}}\right)$ for any $\gamma \in X_{\infty}$. This is because

$$
\left((i \circ \gamma)^{*} \Omega_{X / k[t]}\right) / S \rightarrow\left(\gamma^{*} \Omega_{X^{\prime} / k[t]}\right) / T
$$

is an isomorphism for the inclusion $i: X^{\prime} \rightarrow X$ and an arc $\gamma:$ Spec $k[[t]] \rightarrow X^{\prime}$, where $S$ and $T$ are the torsion parts of $(\gamma \circ i)^{*} \Omega_{X / k[t]}$ and $\gamma^{*} \Omega_{X^{\prime} / k[t]}$, respectively.

2.5. The codimension of cylinders in arc spaces. In this subsection, we prove Proposition 2.17, which is necessary for defining the codimension of cylinders in the arc spaces of $k[t]$ schemes. Proposition 2.17 is a generalization of [DL99, Lemma 4.1] (cf. [EM09, Proposition 4.1]). In their proof, the authors reduce the problem to that for locally complete intersections. The same strategy works in our setting.

First we state Proposition 2.17 for the case of complete intersections with a little generalization. After one replaces $\operatorname{Jac}_{X}$ with the ideal generated by minors of Jacobian matrix, their proof works for non-local complete intersection (non-l.c.i.) varieties.

Lemma 2.15. Let $N$ and $r$ be positive integers with $N \geqslant r$. Let $R=k[t]\left[x_{1}, \ldots, x_{N}\right]$, and let $I=$ $\left(F_{1}, \ldots, F_{r}\right)$ be the ideal generated by elements $F_{1}, \ldots, F_{r} \in R$. We denote by $M=\operatorname{Spec}(R / I)$ the $k[t]$-scheme corresponding to $R / I$. Let $J \subset R$ be the ideal generated by all the $r$-minors of the Jacobian matrix $\left(\partial F_{i} / \partial x_{j}\right)_{1 \leqslant i \leqslant r, 1 \leqslant j \leqslant N}$, and let $\bar{J}=(J+I) / I$. For non-negative integers $m$ and $e$ with $m \geqslant e$, the following hold:

(1) $\psi_{m}\left(\operatorname{Cont}^{e}(\bar{J})\right)=\pi_{m+e, m}\left(\operatorname{Cont}^{e}(\bar{J})_{m+e}\right)$.

(2) The morphism $\pi_{m+1, m}: M_{m+1} \rightarrow M_{m}$ induces a piecewise trivial fibration

$$
\psi_{m+1}\left(\operatorname{Cont}^{e}(\bar{J})\right) \rightarrow \psi_{m}\left(\operatorname{Cont}^{e}(\bar{J})\right)
$$

with fiber $\mathbb{A}^{N-r}$. 


\section{Y. NAKAMURA AND K. ShiBATA}

Proof. The first statement follows from [CNS18, Chapter 1, Lemma 1.3.3].

The second statement for locally complete intersection varieties is proved in the proof of Lemma 4.1 in [DL99] (cf. [EM09, Proposition 4.1]). We can apply the same proof as in [DL99, Lemma 4.1] by replacing Hensel's lemma with [CNS18, Chapter 1, Lemma 1.3.3].

Remark 2.16. In [EM09, Proposition 4.1], the 1.c.i. cases (or, more generally, only the puredimensional cases) are treated. We treat the non-l.c.i. cases in Lemma 2.15 because we will treat such cases in Section 4 (cf. Remark 4.4).

When $X$ is flat over $k[t]$, Proposition 2.17(1) below is proved in [CNS18, Chapter 5, Proposition 2.3.4], and Proposition 2.17(2) is proved in [Seb04, Lemma 4.5.4] (cf. [CNS18, Chapter 5, Theorem 2.3.11]). We note here that Proposition 2.17(2) can be reduced to the flat case by Remark 2.14(3).

Proposition 2.17. Let $X$ be a $k[t]$-scheme with the condition $(\star)_{n}$ from Subsection 2.4. Then there exists a positive integer $c$ such that the following hold for non-negative integers $m$ and $e$ with $m \geqslant c e$ :

(1) $\psi_{m}\left(\operatorname{Cont}^{e}\left(\operatorname{Jac}_{X / k[t]}\right)\right)=\pi_{m+e, m}\left(\operatorname{Cont}^{e}\left(\operatorname{Jac}_{X / k[t]}\right)_{m+e}\right)$.

(2) The morphism $\pi_{m+1, m}: X_{m+1} \rightarrow X_{m}$ induces a piecewise trivial fibration

$$
\psi_{m+1}\left(\operatorname{Cont}^{e}\left(\operatorname{Jac}_{X / k[t]}\right)\right) \rightarrow \psi_{m}\left(\operatorname{Cont}^{e}\left(\operatorname{Jac}_{X / k[t]}\right)\right)
$$

with fiber $\mathbb{A}^{n}$.

Proof. In [Seb04, Lemma 4.5.4] (cf. [CNS18, Chapter 5, Theorem 2.3.11]), assertion (2) is proved for flat formal schemes of finite type of pure relative dimension over $k[[t]]$. Therefore, assertion (2) can be reduced to this result by Remark 2.14(1)-(3). On the other hand, it seems that assertion (1) cannot be easily reduced to the flat case (we can only see that $\psi_{m}\left(\operatorname{Cont}^{e}\left(\mathrm{Jac}_{X / k[t]}\right)\right)=$ $\pi_{m+e+a, m}\left(\operatorname{Cont}^{e}\left(\mathrm{Jac}_{X / k[t]}\right)_{m+e+a}\right)$ by Remark 2.14(3)). However, if we assume the condition $(\star)_{n}$, it turns out that the proof itself is valid for the non-flat case as well. For the reader's convenience, we give a proof below, following the argument in [EM09, Proposition 4.1].

Since the assertion is local on $X$, we may assume that $X \subset \mathbb{A}_{k[t]}^{N}$ is affine. Set $r:=N-n$. Let $R:=k[t]\left[x_{1}, \ldots, x_{N}\right]$, and let $I_{X} \subset R$ be the defining ideal of $X$. Let $f_{1}, \ldots, f_{d}$ be generators of $I_{X}$. For $1 \leqslant i \leqslant d$, we set

$$
F_{i}=\sum_{j=1}^{d} a_{i j} f_{j}
$$

for general $a_{i j} \in k$. Then for each subset $\Lambda \subset\{1, \ldots, d\}$ with $\# \Lambda=r=N-n$, we denote by $M_{\Lambda} \subset \mathbb{A}_{k[t]}^{N}$ the subscheme defined by the ideal $I_{M_{\Lambda}}:=\left(F_{i} \mid i \in \Lambda\right)$ generated by the $F_{i}$ with $i \in \Lambda$. We denote by $J_{\Lambda} \subset R$ the ideal generated by the $r$-minors of the Jacobian matrix $\left(\partial F_{i} / \partial x_{j}\right)_{i \in \Lambda, 1 \leqslant j \leqslant N}$. Set $\bar{J}_{\Lambda}:=\left(J_{\Lambda}+I_{M_{\Lambda}}\right) / I_{M_{\Lambda}}$.

We note that for $\gamma \in X_{\infty}$, we have

$$
\operatorname{ord}_{\gamma}\left(\operatorname{Jac}_{X / k[t]}\right)=\min _{\Lambda} \operatorname{ord}_{\gamma}\left(J_{\Lambda}\right) .
$$

Hence

$$
U_{\Lambda}:=\left\{\gamma \in \operatorname{Cont}^{e}\left(\operatorname{Jac}_{X / k[t]}\right) \mid \operatorname{ord}_{\gamma}\left(J_{\Lambda}\right)=e\right\}
$$

is an open subset of $\operatorname{Cont}^{e}\left(\operatorname{Jac}_{X / k[t]}\right)$ satisfying $\operatorname{Cont}^{e}\left(\operatorname{Jac}_{X / k[t]}\right)=\bigcup_{\Lambda} U_{\Lambda}$. Since $X$ is a closed subscheme of $M_{\Lambda}$, we may identify the arc space $X_{\infty}$ with a closed subset of $\left(M_{\Lambda}\right)_{\infty}$. Under this identification, we have $U_{\Lambda} \subset \operatorname{Cont}^{e}\left(\bar{J}_{\Lambda}\right)$. 


\section{INVERSION OF ADJUNCTION FOR QUOTIENT SINGULARITIES}

Then we claim the following (cf. [EM09, Lemma 4.2]).

Claim 2.18. There exists a positive integer $c_{\Lambda}$ such that the following condition holds for any non-negative integers $m$ and $e$ satisfying $m \geqslant c_{\Lambda} e$ :

- If $\gamma \in \operatorname{Cont}^{e}\left(\bar{J}_{\Lambda}\right) \subset\left(M_{\Lambda}\right)_{\infty}$ satisfies $\psi_{m}(\gamma) \in X_{m}$, then $\gamma \in X_{\infty}$.

Proof. Let $I_{X_{\Lambda}^{\prime}}:=\left(I_{M_{\Lambda}}: I_{X}\right)$, and let $X_{\Lambda}^{\prime} \subset \mathbb{A}_{k[t]}^{N}$ be the corresponding subscheme. For a prime ideal $\mathfrak{p}$ of $R$, we note that $I_{M_{\Lambda}} \subset \mathfrak{p}$ and $I_{X} \not \subset \mathfrak{p}$ imply $I_{X_{\Lambda}^{\prime}}=\left(I_{M_{\Lambda}}: I_{X}\right) \subset \mathfrak{p}$. Therefore, settheoretically, $X_{\Lambda}^{\prime}$ is the union of the irreducible components of $M_{\Lambda}$ which are not contained in $X$. Hence we have $\left(M_{\Lambda}\right)_{\infty}=X_{\infty} \cup\left(X_{\Lambda}^{\prime}\right)_{\infty}$. Since any irreducible component of $X$ has dimension at least $n+1$ and the $a_{i j}$ are general elements of $k$, for any irreducible component $X_{0}$ of $X$, there exists an irreducible component of $M_{0}$ of $M_{\Lambda}$ with $X_{0}=M_{0}$. Therefore, if $M_{\Lambda}$ is smooth at a point $x \in X$, then $X$ is smooth at $x$ and $\mathcal{O}_{X, x}=\mathcal{O}_{M_{\Lambda}, x}$. Hence if $\left(R / I_{M_{\Lambda}}\right)_{\mathfrak{q}}$ is a regular local ring for a prime ideal $\mathfrak{q}$ of $R$ with $I_{X} \subset \mathfrak{q}$, then we have

$$
\left(I_{X_{\Lambda}^{\prime}}\right)_{\mathfrak{q}}=\left(I_{M_{\Lambda}}: I_{X}\right)_{\mathfrak{q}}=\left(\left(I_{M_{\Lambda}}\right)_{\mathfrak{q}}:\left(I_{X}\right)_{\mathfrak{q}}\right)=R_{\mathfrak{q}} .
$$

This implies that $X_{\Lambda}^{\prime} \cap\left(\left(M_{\Lambda}\right)_{\text {reg }} \cap X\right)=\emptyset$. Hence $M_{\Lambda}$ is singular at every point $x \in X \cap X_{\Lambda}^{\prime}$. Here we claim that

(D) $J_{\Lambda} \subset \sqrt{I_{X}+I_{X_{\Lambda}^{\prime}}}$ holds.

Let $J_{\Lambda}^{\prime}$ be the ideal generated by the $r$-minors of the Jacobian matrix with respect to $I_{M_{\Lambda}}=$ $\left(F_{i} \mid i \in \Lambda\right)$ and the derivations $\partial x_{j}$ and $\partial t$. Then by the definition of $J_{\Lambda}$, we have $J_{\Lambda} \subset J_{\Lambda}^{\prime}$. Let $\mathfrak{p}$ be a prime ideal satisfying $I_{X}+I_{X_{\Lambda}^{\prime}} \subset \mathfrak{p}$. Since $\operatorname{ht}\left(I_{M_{\Lambda}} R_{\mathfrak{p}}\right) \leqslant r$ and the $\operatorname{ring} R_{\mathfrak{p}} / I_{M_{\Lambda}} R_{\mathfrak{p}}$ is not regular, it follows by the Jacobian criterion (cf. [Mat89, Theorem 30.4]) that $J_{\Lambda}^{\prime} \subset \mathfrak{p}$, which proves the claim $(\odot)$.

By $(\triangleright)$,

$$
J_{\Lambda}^{c_{\Lambda}} \subset I_{X}+I_{X_{\Lambda}^{\prime}}
$$

holds for some $c_{\Lambda}$. Suppose $\gamma \in \operatorname{Cont}^{e}\left(\bar{J}_{\Lambda}\right) \subset\left(M_{\Lambda}\right)_{\infty}$. Since

$$
\operatorname{ord}_{\gamma}\left(J_{\Lambda}^{c_{\Lambda}}\right)=c_{\Lambda} e<m+1 \leqslant \operatorname{ord}_{\gamma}\left(I_{X}\right),
$$

we have $\operatorname{ord}_{\gamma}\left(I_{X_{\Lambda}^{\prime}}\right) \leqslant c_{\Lambda} e$. Hence $\gamma \notin\left(X_{\Lambda}^{\prime}\right)_{\infty}$, and therefore $\gamma \in X_{\infty}$. This completes the proof of the claim.

Proof of Proposition 2.17, continued. We set $c=\max _{\Lambda} c_{\Lambda}$. Then the assertions (1) and (2) for $X$ follow from the assertions of Lemma 2.15 for $M_{\Lambda}$ by Claim 2.18.

We define cylinders in the arc spaces of $k[t]$-schemes and define their codimension. For a $k[t]$ scheme $X$, a subset $C \subset X_{\infty}$ is called a cylinder if $C=\psi_{m}^{-1}(S)$ holds for some $m \geqslant 0$ and a constructible subset $S \subset X_{m}$.

Definition 2.19. Let $X$ be a $k[t]$-scheme with the condition $(\star \star)_{n}$. Let $C \subset X_{\infty}$ be a cylinder.

(1) Assume that $C \subset \operatorname{Cont}^{e}\left(\operatorname{Jac}_{X / k[t]}\right)$ holds for some $e \in \mathbb{Z}_{\geqslant 0}$. Then we define the codimension of $C$ in $X_{\infty}$ as

$$
\operatorname{codim}(C):=(m+1) n-\operatorname{dim}\left(\psi_{m}(C)\right)
$$

for any sufficiently large $m$. This codimension is well defined by Proposition 2.17.

(2) In general, we define the codimension of $C$ in $X_{\infty}$ as

$$
\operatorname{codim}(C):=\min _{e \in \mathbb{Z}_{\geqslant 0}} \operatorname{codim}\left(C \cap \operatorname{Cont}^{e}\left(\operatorname{Jac}_{X / k[t]}\right)\right) .
$$




\section{Y. NAKAMURA AND K. ShiBATA}

Remark 2.20. The codimension is also well defined for $X$ with $(\star)_{n}$. However, in this case, we may have $X_{\infty} \cap \operatorname{Cont}^{e}\left(\operatorname{Jac}_{X / k[t]}\right)=\emptyset$ for any $e \geqslant 0$, and $\operatorname{codim}(C)=\infty$ may hold for any cylinder $C$. Therefore, we assume $(\star \star)_{n}$ when we discuss the codimension of a cylinder.

Definition 2.21. Let $X$ be a $k[t]$-scheme with the condition $(\star \star)_{n}$. A subset $A \subset X_{\infty}$ is called thin if $A \subset Z_{\infty}$ holds for some closed subscheme $Z$ of $X$ with the condition $(\star \star)_{\ell}$ for some $\ell \leqslant n-1$.

Remark 2.22. The arc space $X_{\infty}$ is never a thin set of $X_{\infty}$ for a $k$-variety $X$. However, $X_{\infty}$ can be a thin set of $X_{\infty}$ for a $k[t]$-scheme $X$ even if we assume the condition $(\star \star)_{n}$. See the example in Remark 5.3.

Lemma 2.23 (cf. [CNS18, Chapter 6, Proposition 2.4.6]). Let $X$ be a $k[t]$-scheme with the condition $(\star)_{n}$, and let $C \subset X_{\infty}$ be a cylinder. If $C$ is thin, then $C \cap \operatorname{Cont}^{e}\left(\operatorname{Jac}_{X / k[t]}\right)=\emptyset$ holds for any $e \geqslant 0$.

Proof. This follows from [CNS18, Chapter 6, Proposition 2.4.6] and Remark 2.14.

Proposition 2.24 ([Seb04, Lemme 4.3.9]). Let $X$ be a scheme of finite type over $k[t]$, and let $C$ be a cylinder in $X_{\infty}$. Then its image $\psi_{m}(C) \subset X_{m}$ is a constructible subset for any $m \geqslant 0$.

Proof. This follows from [Seb04, Lemme 4.3.9] (cf. [CNS18, Chapter 5, Corollary 1.5.7]) and Remark 2.14.

Proposition 2.25 (cf. [Seb04, Théorème 6.3.5]). Let $X$ be a $k[t]$-scheme with the condition $(\star \star)_{n}$, and let $C$ be a cylinder in $X_{\infty}$. Let $\left\{C_{\lambda}\right\}_{\lambda \in \Lambda}$ be a set of countably many disjoint subcylinders $C_{\lambda} \subset C$. If $C \backslash\left(\bigsqcup_{\lambda \in \Lambda} C_{\lambda}\right) \subset X_{\infty}$ is a thin set, then it follows that

$$
\operatorname{codim}(C)=\min _{\lambda \in \Lambda} \operatorname{codim}\left(C_{\lambda}\right) \text {. }
$$

Proof. This follows from [CNS18, Chapter 6, Lemma 3.4.1 and Example 3.5.2].

Lemma 2.26. Let $X$ be a variety over Spec $k[t]$ which dominates Spec $k[t]$ and has relative dimension $n$. Suppose that $X$ is smooth over $k$. Then there exists a non-negative integer $\ell$ such that the following hold:

(1) We have $\operatorname{ord}_{\gamma}\left(\operatorname{Jac}_{X / k[t]}\right) \leqslant \ell$ for every arc $\gamma \in X_{\infty}$.

(2) We have $\psi_{m}\left(X_{\infty}\right)=\pi_{m+\ell, m}\left(X_{m+\ell}\right)$ for every $m \geqslant \ell$.

(3) For any $m \geqslant \ell$, the morphism $\pi_{m+1, m}$ induces a piecewise trivial fibration $\psi_{m+1}\left(X_{\infty}\right) \rightarrow$ $\psi_{m}\left(X_{\infty}\right)$ with fiber $\mathbb{A}^{n}$.

Proof. Since $X$ is smooth over $k$, by the generic smoothness, $X$ is smooth over Spec $k[t]$ outside finitely many closed points. Therefore, we have an inclusion of ideals $\left(t^{\ell}\right) \subset \mathrm{Jac}_{X / k[t]}$ in a neighborhood of $t=0$ for some $\ell \geqslant 0$. Hence $\operatorname{ord}_{\gamma}\left(\operatorname{Jac}_{X / k[t]}\right) \leqslant \ell$ holds for any arc $\gamma \in X_{\infty}$. Then assertions (2) and (3) follow from Lemma 2.15.

Lemma 2.27. Let $f: Y \rightarrow X$ be a proper birational $k[t]$-morphism of $k[t]$-varieties $X$ and $Y$. Suppose that $Y$ is smooth over $k$. Let $C \subset X_{\infty}$ be a cylinder. If $C$ is a thin set of $X_{\infty}$, then $f_{\infty}^{-1}(C)=\emptyset$.

Proof. We may assume that $X$ dominates Spec $k[t]$. Let $n$ be the relative dimension of $X$. Since $C$ is a thin set, there exists a closed subset $Z \subsetneq X$ such that $C \subset Z_{\infty}$. Set $Z^{\prime}:=f^{-1}(Z)$. Then we have $f_{\infty}^{-1}(C) \subset Z_{\infty}^{\prime}$ (cf. Lemma 2.28(2)). Since dominant components of $Z^{\prime}$ have relative dimension at most $n-1$, the cylinder $f_{\infty}^{-1}(C)$ is also a thin set. By Lemmas 2.23 and 2.26(1), we have $f_{\infty}^{-1}(C)=\emptyset$. 
Lemma 2.28. (1) Let $Z \subset X$ be a closed subscheme of a $k[t]$-scheme $X$ of finite type. Then the induced map $f_{\infty}: Z_{\infty} \rightarrow X_{\infty}$ is a closed immersion.

(2) Moreover, for a $k[t]$-morphism $f: Y \rightarrow X$, it follows that $\left(f^{-1}(Z)\right)_{\infty} \simeq f_{\infty}^{-1}\left(Z_{\infty}\right)$.

Proof. The assertions follow from the same argument for $k$-varieties (cf. [EM09, Remarks 2.7 and 2.8]).

2.6. Fundamental properties of the arc spaces of $\boldsymbol{k}[\boldsymbol{t}]$-schemes. In this subsection, we prove Proposition 2.33, which is a generalization of [DL02, Lemma 1.17] to $k[t]$-schemes with the condition $(\star \star)_{n}$. Actually, in [DL02, Remark 1.19], it is mentioned that [DL02, Lemma 1.17] can be generalized to separated reduced schemes of finite type over $k[t]$. In [Yas19, Lemma 10.20], Yasuda proves Proposition 2.33 in a more general setting. For the reader's convenience, we give a proof of Proposition 2.33 following the argument in [EM09].

Proposition 2.29. Let $X$ be a $k[t]$-scheme with the condition $(\star)_{n}$. Let $p$ and $m$ be non-negative integers with $2 p+1 \geqslant m \geqslant p$.

(1) Let $\gamma \in X_{p}$ with $\pi_{m, p}^{-1}(\gamma) \neq \emptyset$. Then scheme-theoretically, we have

$$
\pi_{m, p}^{-1}(\gamma) \simeq \operatorname{Hom}_{k[t] /\left(t^{p+1}\right)}\left(\gamma^{*} \Omega_{X / k[t]},\left(t^{p+1}\right) /\left(t^{m+1}\right)\right) .
$$

(2) Let $\gamma^{\prime} \in X_{\infty}$, and let $e:=\operatorname{ord}_{\gamma^{\prime}}\left(\operatorname{Jac}_{X / k[t]}\right)$. Let $c$ be a positive integer appearing in Proposition 2.17. Let $T$ be the torsion part of $\gamma^{\prime *} \Omega_{X}$. Suppose $2 p+1-e \geqslant m \geqslant c e$ and $p \geqslant e$. For $\gamma=\psi_{p}\left(\gamma^{\prime}\right)$, it follows that

$$
\begin{aligned}
& \pi_{m, p}^{-1}(\gamma) \cap \psi_{m}\left(\operatorname{Cont}^{e}\left(\operatorname{Jac}_{X / k[t]}\right)\right)=\pi_{m+e, m}\left(\pi_{m+e, p}^{-1}(\gamma)\right) \\
& \quad \simeq \operatorname{Hom}_{k[t] /\left(t^{p+1}\right)}\left(\left(\gamma^{\prime *} \Omega_{X / k[t]}\right) / T \otimes_{k[[t]]} k[t] /\left(t^{p+1}\right),\left(t^{p+1}\right) /\left(t^{m+1}\right)\right) .
\end{aligned}
$$

Proof. We first prove assertion (1). We may assume $X=\operatorname{Spec} A$. Let $\gamma^{*}: A \rightarrow k[t] /\left(t^{p+1}\right)$ be the $k[t]$-ring homomorphism corresponding to $\gamma$. Suppose $\alpha \in \pi_{m, p}^{-1}(\gamma)$ and that $\alpha^{*}: A \rightarrow k[t] /\left(t^{m+1}\right)$ is the $k[t]$-ring homomorphism corresponding to $\alpha$. Then we have

$$
\pi_{m, p}^{-1}(\gamma) \simeq \operatorname{Der}_{k[t]}\left(A,\left(t^{p+1}\right) /\left(t^{m+1}\right)\right), \quad \beta \mapsto \beta^{*}-\alpha^{*} ;
$$

here $\left(t^{p+1}\right) /\left(t^{m+1}\right)$ has an $A$-module structure via $\gamma^{*}$ (cf. [EM09, Proposition 4.4]). Then the assertion follows from the isomorphisms

$$
\begin{aligned}
\operatorname{Der}_{k[t]}\left(A,\left(t^{p+1}\right) /\left(t^{m+1}\right)\right) & \simeq \operatorname{Hom}_{A}\left(\Omega_{A / k[t]},\left(t^{p+1}\right) /\left(t^{m+1}\right)\right) \\
& \simeq \operatorname{Hom}_{k[t] /\left(t^{p+1}\right)}\left(\gamma^{*} \Omega_{X / k[t]},\left(t^{p+1}\right) /\left(t^{m+1}\right)\right) .
\end{aligned}
$$

We now prove assertion (2). Note that

$$
\begin{aligned}
\gamma^{*} \Omega_{X / k[t]} & =\gamma^{\prime *} \Omega_{X / k[t]} \otimes_{k[[t]]} k[t] /\left(t^{p+1}\right) \\
& \simeq\left(\left(\gamma^{\prime *} \Omega_{X / k[t]}\right) / T \otimes_{k[[t]]} k[t] /\left(t^{p+1}\right)\right) \oplus\left(T \otimes_{k[[t]]} k[t] /\left(t^{p+1}\right)\right) .
\end{aligned}
$$

Since $T$ is the form of $\bigoplus_{i} k[t] /\left(t^{e_{i}}\right)$ with $\sum_{i} e_{i}=e$ and, in particular, $e_{i} \leqslant e \leqslant p$, it follows that $T \otimes_{k[[t]]} k[t] /\left(t^{p+1}\right) \simeq \bigoplus_{i} k[t] /\left(t^{e_{i}}\right)$. Note also that $\lambda: \gamma^{*} \Omega_{X / k[t]} \rightarrow\left(t^{p+1}\right) /\left(t^{m+1}\right)$ lifts to $\gamma^{*} \Omega_{X / k[t]} \rightarrow\left(t^{p+1}\right) /\left(t^{m+e+1}\right)$ if and only if $\lambda\left(T \otimes k[t] /\left(t^{p+1}\right)\right)=0$ holds (Lemma 2.30(2)). This equivalence and assertion (1) show that

$$
\begin{aligned}
\pi_{m+e, m}\left(\pi_{m+e, p}^{-1}(\gamma)\right) & \simeq \operatorname{Im}\left(\begin{array}{c}
\operatorname{Hom}_{k[t] /\left(t^{p+1}\right)}\left(\gamma^{*} \Omega_{X / k[t]},\left(t^{p+1}\right) /\left(t^{m+e+1}\right)\right) \\
\longrightarrow \operatorname{Hom}_{k[t] /\left(t^{p+1}\right)}\left(\gamma^{*} \Omega_{X / k[t]},\left(t^{p+1}\right) /\left(t^{m+1}\right)\right)
\end{array}\right) \\
& \simeq \operatorname{Hom}_{k[t] /\left(t^{p+1}\right)}\left(\left(\gamma^{\prime *} \Omega_{X / k[t]}\right) / T \otimes_{k[t]]} k[t] /\left(t^{p+1}\right),\left(t^{p+1}\right) /\left(t^{m+1}\right)\right) .
\end{aligned}
$$




\section{Y. NAKAmura AND K. Shibata}

Since $\operatorname{ord}_{\gamma}\left(\operatorname{Jac}_{X / k[t]}\right)=e$, it follows from Proposition 2.17(1) that

$$
\pi_{m+e, m}\left(\pi_{m+e, p}^{-1}(\gamma)\right)=\pi_{m, p}^{-1}(\gamma) \cap \psi_{m}\left(\operatorname{Cont}^{e}\left(\operatorname{Jac}_{X / k[t]}\right)\right) .
$$

This completes the proof.

Lemma 2.30. Let $m, p, \ell$ be non-negative integers with $m \geqslant p$. Then the following hold:

(1) The $k$-vector space $\operatorname{Hom}_{k[t]}\left(k[t] /\left(t^{\ell}\right),\left(t^{p+1}\right) /\left(t^{m+1}\right)\right)$ is isomorphic to $\mathbb{A}^{\ell}$ if $\ell \leqslant m-p$; otherwise, it is isomorphic to $\mathbb{A}^{m-p}$.

(2) If $\ell \leqslant e$, only the zero map $k[t] /\left(t^{\ell}\right) \rightarrow\left(t^{p+1}\right) /\left(t^{m+1}\right)$ can lift to $k[t] /\left(t^{\ell}\right) \rightarrow\left(t^{p+1}\right) /\left(t^{m+e+1}\right)$.

Proof. The proof is straightforward.

For an $\operatorname{arc} \delta$, we denote by $\delta_{m}$ its image in the space of the $m$ th jets.

Lemma 2.31. Let $X$ and $Y$ be $k[t]$-schemes with the condition $(\star)_{n}$, and let $f: Y \rightarrow X$ be a morphism over $k[t]$. Let $e, e^{\prime}, e^{\prime \prime}, q \in \mathbb{Z}_{\geqslant 0}$. Let $c_{X}$ and $c_{Y}$ be positive integers for $X$ and $Y$ appearing in Proposition 2.17. Suppose $\max \left\{e+e^{\prime}, e+e^{\prime \prime}, c_{X} e^{\prime}, c_{Y} e^{\prime \prime}\right\} \leqslant q-e$. Let $\alpha \in \mathrm{Cont}^{e^{\prime}}\left(\mathrm{Jac}_{X / k[t]}\right)$ and $\beta \in \operatorname{Cont}^{e^{\prime \prime}}\left(\operatorname{Jac}_{Y / k[t]}\right)$ with $f_{q}\left(\beta_{q}\right)=\alpha_{q}$ and $\operatorname{ord}_{\beta}\left(\operatorname{jac}_{f}\right)=e$. Then there is a $\delta \in \operatorname{Cont}^{e^{\prime \prime}}\left(\operatorname{Jac}_{Y / k[t]}\right)$ with $f_{q+1}\left(\delta_{q+1}\right)=\alpha_{q+1}$ such that $\beta_{q-e}=\delta_{q-e}$ and $\operatorname{ord}_{\delta}\left(\operatorname{jac}_{f}\right)=e$.

Proof. Let $S$ and $T$ be the torsion parts of $\alpha^{*} \Omega_{X / k[t]}$ and $\beta^{*} \Omega_{Y / k[t]}$, respectively. By Proposition 2.29(2), we have

$$
\begin{aligned}
& \pi_{q+1, q-e}^{-1}\left(\alpha_{q-e}\right) \cap \psi_{q+1}\left(\operatorname{Cont}^{e^{\prime}}\left(\operatorname{Jac}_{X / k[t]}\right)\right) \\
& \quad \simeq \operatorname{Hom}_{k[t] /\left(t^{q-e+1}\right)}\left(\left(\alpha^{*} \Omega_{X / k[t]}\right) / S \otimes_{k[t]]} k[t] /\left(t^{q-e+1}\right),\left(t^{q-e+1}\right) /\left(t^{q+2}\right)\right)
\end{aligned}
$$

and

$$
\begin{aligned}
& \pi_{q+1, q-e}^{-1}\left(\beta_{q-e}\right) \cap \psi_{q+1}\left(\operatorname{Cont}^{e^{\prime \prime}}\left(\operatorname{Jac}_{Y / k[t]}\right)\right) \\
& \quad \simeq \operatorname{Hom}_{k[t] /\left(t^{q-e+1}\right)}\left(\left(\beta^{*} \Omega_{Y / k[t]}\right) / T \otimes_{k[[t]]} k[t] /\left(t^{q-e+1}\right),\left(t^{q-e+1}\right) /\left(t^{q+2}\right)\right) .
\end{aligned}
$$

We may assume that $\beta_{q+1}$ corresponds to the zero map via this isomorphisms. Let

$$
w: \alpha^{*} \Omega_{X / k[t]} / S \otimes_{k[[t]]} k[t] /\left(t^{q-e+1}\right) \rightarrow\left(t^{q-e+1}\right) /\left(t^{q+2}\right)
$$

be the morphism corresponding to $\alpha_{q+1}$ via this isomorphism. Then it is sufficient to show the existence of a morphism

$$
u: \beta^{*} \Omega_{Y / k[t]} / T \otimes_{k[[t]]} k[t] /\left(t^{q-e+1}\right) \rightarrow\left(t^{q-e+1}\right) /\left(t^{q+2}\right)
$$

such that $u \circ h_{q-e}=w$, where

$$
h_{q-e}: \alpha^{*} \Omega_{X / k[t]} / S \otimes_{k[[t]]} k[t] /\left(t^{q-e+1}\right) \rightarrow \beta^{*} \Omega_{Y / k[t]} / T \otimes_{k[[t]]} k[t] /\left(t^{q-e+1}\right)
$$

is the natural morphism.

By the definition of $\operatorname{ord}_{\beta}\left(\mathrm{jac}_{f}\right)$, we have

$$
\operatorname{Coker}\left(\beta^{\prime *} \Omega_{X / k[t]} \rightarrow \beta^{*} \Omega_{Y / k[t]} / T\right) \simeq k[t] /\left(t^{a_{1}}\right) \oplus \cdots \oplus k[t] /\left(t^{a_{n}}\right)
$$

with $a_{i} \geqslant 0$ and $\sum_{i} a_{i}=e$, where $\beta^{\prime}:=f_{\infty}(\beta)$. Since $\beta_{q-e}^{\prime}=\alpha_{q-e}$ and $a_{i} \leqslant q-e+1$, we have

$$
\operatorname{Coker}\left(h_{q-e}\right) \simeq k[t] /\left(t^{a_{1}}\right) \oplus \cdots \oplus k[t] /\left(t^{a_{n}}\right) .
$$

Hence we can regard $h_{q-e}$ as the morphism given by the diagonal matrix with entries $t^{a_{1}}, \ldots, t^{a_{n}}$. Furthermore, since $\alpha_{q}=f_{q}\left(\beta_{q}\right)$, it follows that $\operatorname{Im}(w) \subset\left(t^{q+1}\right) /\left(t^{q+2}\right)$. Since $q \geqslant e+a_{i}$ holds for each $i$, we can find a desired $u$. 
Lemma 2.32. Let $X$ and $Y$ be $k[t]$-schemes with the condition $(\star)_{n}$, and let $f: Y \rightarrow X$ be a morphism over $k[t]$. Let $e, e^{\prime}, e^{\prime \prime}, m \in \mathbb{Z}_{\geqslant 0}$. Let $c_{X}$ and $c_{Y}$ be positive integers for $X$ and $Y$ appearing in Proposition 2.17. Suppose $\max \left\{e+e^{\prime}, e+e^{\prime \prime}, c_{X} e^{\prime}, c_{Y} e^{\prime \prime}\right\} \leqslant m-e$. Let $\alpha \in$ $\operatorname{Cont}^{e^{\prime}}\left(\mathrm{Jac}_{X / k[t]}\right)$ and $\beta \in \operatorname{Cont}^{e^{\prime \prime}}\left(\mathrm{Jac}_{Y / k[t]}\right)$ with $f_{m}\left(\beta_{m}\right)=\alpha_{m}$ and $\operatorname{ord}_{\beta}\left(\mathrm{jac}_{f}\right)=e$. Then there is a $\delta \in \operatorname{Cont}^{e^{\prime \prime}}\left(\operatorname{Jac}_{Y / k[t]}\right)$ with $\beta_{m-e}=\delta_{m-e}$ such that $f_{\infty}(\delta)=\alpha$ and $\operatorname{ord}_{\delta}\left(\operatorname{jac}_{f}\right)=e$.

Proof. By Lemma 2.31, we can construct recursively $\delta^{(q)} \in \operatorname{Cont}^{e^{\prime \prime}}\left(\mathrm{Jac}_{Y / k[t]}\right)$ for $q \geqslant m$ such that $\delta^{(m)}=\beta, \delta_{q-e}^{(q+1)}=\delta_{q-e}^{(q)}$ and $f_{q}\left(\delta_{q}^{(q)}\right)=\alpha_{q}$ for every $q \geqslant m$. The sequence $\left(\delta_{q-e}^{(q)}\right)_{q \in \mathbb{Z} \geqslant m}$ defines an element $\delta \in \operatorname{Cont}^{e^{\prime \prime}}\left(\mathrm{Jac}_{Y / k[t]}\right)$ such that $\delta_{q-e}=\delta_{q-e}^{(q)}$ for every $q \geqslant m$. By the construction of $\delta$, it follows that $\beta_{m-e}=\delta_{m-e}$ and $f_{\infty}(\delta)=\alpha$.

When $Y$ is smooth over $k[t]$, Proposition 2.33 below is proved in [Seb04, Lemma 7.1.3] (cf. [CNS18, Chapter 5, Theorem 3.2.2]). In [Yas19, Lemmas 10.19 and 10.20], Yasuda proves Proposition 2.33 in a more general setting (for formal Deligne-Mumford stacks of arbitrary characteristic).

Proposition 2.33 (cf. [DL02, Lemma 1.17, Remark 1.19]). Let $X$ and $Y$ be $k[t]$-schemes with the condition $(\star \star)_{n}$, and let $f: Y \rightarrow X$ be a morphism over $k[t]$. Let $e, e^{\prime}, e^{\prime \prime} \in \mathbb{Z}_{\geqslant 0}$. Let $B$ be a cylinder of $Y_{\infty}$, and let $A=f_{\infty}(B)$. Assume

$$
B \subset \operatorname{Cont}^{e^{\prime \prime}}\left(\operatorname{Jac}_{Y / k[t]}\right) \cap \operatorname{Cont}^{e}\left(\mathrm{jac}_{f}\right), \quad A \subset \operatorname{Cont}^{e^{\prime}}\left(\operatorname{Jac}_{X / k[t]}\right) .
$$

Then $A$ is a cylinder of $X_{\infty}$. Moreover, if $\left.f_{\infty}\right|_{B}$ is injective, then it follows that

$$
\operatorname{codim}(B)+e=\operatorname{codim}(A) .
$$

Proof. The second statement is obtained by specializing [Yas19, Lemma 10.20] to the case where $\Phi=\Psi=k$ and $\mathcal{Y}$ and $\mathcal{X}$ are the formal schemes over $k[[t]]$ associated with $Y$ and $X$, respectively (cf. Remark 2.14). For the reader's convenience, we give a proof in our setting below.

First, we prove that $A$ is a cylinder. Let $B_{m} \subset Y_{m}$ be a constructible subset such that $B=\psi_{m}^{-1}\left(B_{m}\right)$. By Proposition 2.24, we may assume $B_{m}=\psi_{m}(B)$. Furthermore, we may assume that $m$ is sufficiently large, and hence $B=\psi_{m-e}^{-1}\left(\pi_{m, m-e}\left(B_{m}\right)\right)$ also holds. It is enough to show that $A=\psi_{m}^{-1}\left(A_{m}\right)$ for $A_{m}=f_{m}\left(B_{m}\right)$. The inclusion $A \subset \psi_{m}^{-1}\left(A_{m}\right)$ is obvious. We shall show the opposite inclusion. Suppose that $\alpha \in X_{\infty}$ satisfies $\psi_{m}(\alpha) \in A_{m}$. Then by the definition of $A_{m}$, there exists a $\beta \in Y_{\infty}$ such that its image in $X_{m}$ coincides with $\alpha_{m}$. Therefore, by Lemma 2.32, there exists a $\gamma \in Y_{\infty}$ such that $f_{\infty}(\gamma)=\alpha$ and $\psi_{m-e}(\gamma)=\psi_{m-e}(\beta)$. Since $\gamma \in \psi_{m-e}^{-1}\left(\pi_{m, m-e}\left(\beta_{m}\right)\right) \subset B$, it follows that $\alpha \in f_{\infty}(B)=A$. Therefore, $A$ is a cylinder.

Next we shall prove that $\operatorname{codim}(B)+e=\operatorname{codim}(A)$. For this, it is sufficient to show that $\operatorname{dim}\left(f_{m}^{-1}\left(\alpha_{m}\right) \cap B_{m}\right)=e$ for each $\alpha_{m} \in A_{m}$. Let $\alpha \in A$ be a lift of $\alpha_{m}$, and let $\beta \in B$ be an arc satisfying $f_{\infty}(\beta)=\alpha$.

We claim that

$$
\pi_{m, m-e}\left(f_{m}^{-1}\left(\alpha_{m}\right) \cap B_{m}\right)=\left\{\beta_{m-e}\right\} .
$$

Take $\beta_{m}^{\prime} \in f_{m}^{-1}\left(\alpha_{m}\right) \cap B_{m}$. Then by Lemma 2.32, there exists a $\gamma \in B$ such that $\gamma_{m-e}=$ $\pi_{m, m-e}\left(\beta_{m}^{\prime}\right)$ and $f_{\infty}(\gamma)=\alpha$. Since $\left.f_{\infty}\right|_{B}$ is injective, it follows that $\beta=\gamma$. Therefore, we have $\pi_{m, m-e}\left(\beta_{m}^{\prime}\right)=\beta_{m-e}$.

Hence we have

$$
\begin{aligned}
f_{m}^{-1}\left(\alpha_{m}\right) \cap B_{m} & =f_{m}^{-1}\left(\alpha_{m}\right) \cap \pi_{m, m-e}^{-1}\left(\beta_{m-e}\right) \\
& \subset \pi_{m, m-e}^{-1}\left(\beta_{m-e}\right) \cap \psi_{m}\left(\operatorname{Cont}^{e \prime}\left(\operatorname{Jac}_{Y / k[t]}\right)\right) .
\end{aligned}
$$




\section{Y. NAKAMURA AND K. ShiBATA}

Therefore, by Proposition 2.29, $f_{m}^{-1}\left(\alpha_{m}\right) \cap B_{m}$ is isomorphic to the kernel of

$$
\begin{aligned}
& \operatorname{Hom}_{k[t] /\left(t^{m-e+1}\right)}\left(\left(\beta^{*} \Omega_{Y / k[t]}\right) / T \otimes_{k[[t]]} k[t] /\left(t^{m-e+1}\right),\left(t^{m-e+1}\right) /\left(t^{m+1}\right)\right) \\
& \quad \rightarrow \operatorname{Hom}_{k[t] /\left(t^{m-e+1}\right)}\left(\left(\alpha^{*} \Omega_{X / k[t]}\right) / S \otimes_{k[[t]]} k[t] /\left(t^{m-e+1}\right),\left(t^{m-e+1}\right) /\left(t^{m+1}\right)\right),
\end{aligned}
$$

where $S$ and $T$ are the torsion parts of $\alpha^{*} \Omega_{X / k[t]}$ and $\beta^{*} \Omega_{Y / k[t]}$, respectively. By the definition of $\operatorname{ord}_{\beta}\left(\mathrm{jac}_{f}\right)$, this is isomorphic to

$$
\operatorname{Hom}_{k[t] /\left(t^{m-e+1}\right)}\left(\bigoplus_{i} k[t] /\left(t^{a_{i}}\right) \otimes_{k[[t]]} k[t] /\left(t^{m-e+1}\right),\left(t^{m-e+1}\right) /\left(t^{m+1}\right)\right),
$$

with $a_{i}>0$ and $\sum_{i} a_{i}=e$. This is isomorphic to $\mathbb{A}^{e}$ by Lemma 2.30(1). This completes the proof.

The following lemma is a generalization of Lemma 8.4 in [EM09] to $k[t]$-schemes. This lemma plays an important role in the proof of Theorem 5.1.

Lemma 2.34. Let $A=\operatorname{Spec} k[t]\left[x_{1}, \ldots, x_{N}\right]$, and let $X \subset A$ be a closed subscheme with the condition $(\star \star)_{n}$. Suppose $c:=N-n \geqslant 0$. We denote by $I_{X} \subset k[t]\left[x_{1}, \ldots, x_{N}\right]$ the defining ideal of $X$ in $A$. Suppose that $I_{X}$ is generated by $c$ elements $f_{1}, \ldots, f_{c} \in k[t]\left[x_{1}, \ldots, x_{N}\right]$. Let $C \subset A_{\infty}$ be an irreducible locally closed cylinder. If

- $C \subset \bigcap_{i=1}^{c}$ Cont $^{\geqslant} d_{i}\left(f_{i}\right)$ and

- $C \cap X_{\infty} \cap \operatorname{Cont}^{e}\left(\operatorname{Jac}_{X / k[t]}\right) \neq \emptyset$

hold for some $d_{i} \geqslant 0$ and $e \geqslant 0$, then it follows that

$$
\operatorname{codim}_{X_{\infty}}\left(C \cap X_{\infty}\right) \leqslant \operatorname{codim}_{A_{\infty}}(C)+e-\sum_{i=1}^{c} d_{i}
$$

Proof. The same proof as in [EM09, Lemma 8.4] works by replacing $\mathrm{Jac}_{M}$ in [EM09] with $\mathrm{Jac}_{X / k[t]}$. We note that [EM09, Proposition 4.4(ii)], which is used in the proof, is still true for our $k[t]$-scheme $X$ :

- Let $p, m$ and $e$ be non-negative integers with $2 p \geqslant m \geqslant p+e$. Let $\gamma \in X_{p}$ with $\pi_{m, p}^{-1}(\gamma) \neq \emptyset$ and $\operatorname{ord}_{\gamma}\left(\operatorname{Jac}_{X / k[t]}\right)=e$. Then it follows that $\pi_{m, p}^{-1}(\gamma) \simeq \mathbb{A}^{e+(m-p) n}$.

In [EM09, Proposition 4.4(ii)], the assertion above is proved for l.c.i. varieties. This l.c.i. assumption is used only for proving $\operatorname{Fitt}^{n-1}\left(\Omega_{X / k}\right)=0$. In our case, Fitt ${ }^{n-1}\left(\Omega_{X / k[t]}\right)=0$ holds by the assumption that $I_{X}$ is generated by $c=N-n$ elements.

2.7. Dimension of the arc spaces of quotient varieties. In this subsection, we prove Proposition 2.35, which is a generalization of [DL02, Lemma 3.5] to singular $k[t]$-schemes.

Let $Y$ be a $k[t]$-scheme with the condition $(\star \star)_{n}$. Suppose that a finite group acts on $Y$ over $k[t]$. We denote its quotient by $X:=Y / G$ and the quotient map by $h: Y \rightarrow X$. Let $B \subset Y_{\infty}$ be a $G$-invariant cylinder and $A=h_{\infty}(B)$. Let $e, e^{\prime}, e^{\prime \prime} \in \mathbb{Z}_{\geqslant 0}$. Assume

$$
B \subset \operatorname{Cont}^{e^{\prime \prime}}\left(\operatorname{Jac}_{Y / k[t]}\right) \cap \operatorname{Cont}^{e}\left(\operatorname{jac}_{f}\right), \quad A \subset \operatorname{Cont}^{e^{\prime}}\left(\operatorname{Jac}_{X / k[t]}\right) .
$$


We have the following diagram:

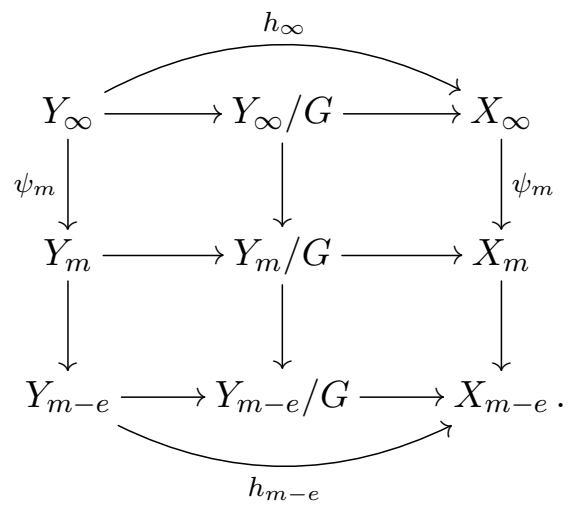

Proposition 2.35 (cf. [DL02, Lemma 3.5]). In the setting above, the following hold:

(1) The image $A$ is a cylinder of $X_{\infty}$.

(2) $\operatorname{codim}(B)+e=\operatorname{codim}(A)$.

Proof. The second statement is obtained by specializing [Yas19, Lemma 10.20] to the case where $\Phi=\Psi=k, g:[\mathcal{Y} / G] \rightarrow \mathcal{X}$ and $C=B / G$, where $\mathcal{Y}$ and $\mathcal{X}$ are the formal schemes over $k[[t]]$ associated with $Y$ and $X$, respectively (cf. Remark 2.14). We note that $\left|\mathrm{J}_{\infty}([\mathcal{Y} / \mathrm{G}])\right|$ is equal to $Y_{\infty} / G$ in the notation of [Yas19], and, furthermore, $\left.g_{\infty}\right|_{B / G}$ is injective. For the reader's convenience, we give a proof in our setting below.

By the same argument as that of the proof of Proposition 2.33, we can take a sufficiently large $m$ and a constructible subset $B_{m} \subset Y_{m}$, and $A_{m}=h_{m}\left(B_{m}\right)$, such that

$$
A=\psi_{m}^{-1}\left(A_{m}\right), \quad B=\psi_{m-e}^{-1}\left(\pi_{m, m-e}\left(B_{m}\right)\right) .
$$

In particular, $A$ is a cylinder of $X_{\infty}$.

In order to prove $\operatorname{codim}(B)+e=\operatorname{codim}(A)$, it is sufficient to show that $\operatorname{dim}\left(h_{m}^{-1}\left(\alpha_{m}\right) \cap B_{m}\right)$ $=e$ for each $\alpha_{m} \in A_{m}$. Let $\alpha \in A$ be a lift of $\alpha_{m}$, and let $\beta \in B$ be an arc satisfying $h_{\infty}(\beta)=\alpha$.

We claim that

- every arc in $\pi_{m, m-e}\left(h_{m}^{-1}\left(\alpha_{m}\right) \cap B_{m}\right)$ has the same image in $Y_{m-e} / G$.

Take $\beta_{m}^{\prime} \in h_{m}^{-1}\left(\alpha_{m}\right) \cap B_{m}$. Then by Lemma 2.32, there exists a $\delta \in B$ such that $\delta_{m-e}=$ $\pi_{m, m-e}\left(\beta_{m}^{\prime}\right)$ and $h_{\infty}(\delta)=\alpha$. Since $Y_{\infty} / G \rightarrow X_{\infty}$ is injective (cf. [GW10, Proposition 12.27(2)]), it follows that $\beta$ and $\delta$ have the same image in $Y_{\infty} / G$. Therefore, the image of $\beta_{m}^{\prime}$ in $Y_{m-e} / G$ coincides with that of $\beta$.

By the claim above, we have

$$
\begin{aligned}
h_{m}^{-1}\left(\alpha_{m}\right) \cap B_{m} & =h_{m}^{-1}\left(\alpha_{m}\right) \cap \bigcup_{\gamma \in G} \pi_{m, m-e}^{-1}\left(\gamma \cdot \beta_{m-e}\right) \\
& \subset \bigcup_{\gamma \in G}\left(\pi_{m, m-e}^{-1}\left(\gamma \cdot \beta_{m-e}\right) \cap \psi_{m}\left(\operatorname{Cont}^{\prime \prime}\left(\operatorname{Jac}_{Y / k[t]}\right)\right)\right) .
\end{aligned}
$$

Therefore, by Proposition 2.29, the intersection $h_{m}^{-1}\left(\alpha_{m}\right) \cap B_{m}$ is isomorphic to the union of the kernels $K_{\gamma}$ of

$$
\begin{aligned}
& \operatorname{Hom}_{k[t] /\left(t^{m-e+1}\right)}\left(\left((\gamma \cdot \beta)^{*} \Omega_{Y / k[t]}\right) / T_{\gamma} \otimes_{k[[t]]} k[t] /\left(t^{m-e+1}\right),\left(t^{m-e+1}\right) /\left(t^{m+1}\right)\right) \\
& \quad \rightarrow \operatorname{Hom}_{k[t] /\left(t^{m-e+1}\right)}\left(\left(\alpha^{*} \Omega_{X / k[t]}\right) / S \otimes_{k[[t]]} k[t] /\left(t^{m-e+1}\right),\left(t^{m-e+1}\right) /\left(t^{m+1}\right)\right)
\end{aligned}
$$


where $S$ and $T_{\gamma}$ are the torsion parts of $\alpha^{*} \Omega_{X / k[t]}$ and $(\gamma \cdot \beta)^{*} \Omega_{Y / k[t]}$, respectively. By the definition of $\operatorname{ord}_{\beta}\left(\mathrm{jac}_{h}\right)$, the kernel $K_{\gamma}$ is isomorphic to

$$
\operatorname{Hom}_{k[t] /\left(t^{m-e+1}\right)}\left(\bigoplus_{i} k[t] /\left(t^{a_{i}}\right) \otimes_{k[[t]]} k[t] /\left(t^{m-e+1}\right),\left(t^{m-e+1}\right) /\left(t^{m+1}\right)\right),
$$

with $a_{i}>0$ and $\sum_{i} a_{i}=e$. Therefore, $K_{\gamma} \simeq \mathbb{A}^{e}$, and hence $\operatorname{dim}\left(h_{m}^{-1}\left(\alpha_{m}\right) \cap B_{m}\right)=e$, which completes the proof.

\section{Denef and Loeser's theory for quotient varieties}

In this section, first we review the theory of the arc space of quotient varieties established by Denef and Loeser [DL02] in more detail (Propositions 3.4 and 3.7). In Subsection 3.3, we study quotients of singular varieties and make a statement analogous to Proposition 3.7 for this setting (Proposition 3.8).

3.1. Lifting property of arcs on quotient varieties. Let $\bar{X}$ be a variety over $k$, and let $G$ be a finite group with order $d$ acting on $\bar{X}$. Let $q: \bar{X} \rightarrow X:=\bar{X} / G$ be the quotient morphism. Let $Z \subset X$ be the minimal closed subset such that $q$ is étale outside $Z$. Set

$$
X_{\infty}^{\mathrm{g}}:=X_{\infty} \backslash Z_{\infty}, \quad \bar{X}_{\infty}^{1 / d}:=\operatorname{Hom}_{k}\left(\operatorname{Spec} k\left[\left[t^{1 / d}\right]\right], \bar{X}\right)
$$

Lemma 3.1. Any $\varphi \in X_{\infty}^{\mathrm{g}}$ lifts to $\bar{X}_{\infty}^{1 / d}$. That is, the composition Spec $k\left[\left[t^{1 / d}\right]\right] \rightarrow$ Spec $k[[t]] \stackrel{\varphi}{\rightarrow} X$ factors through $\bar{X}$. Moreover, $\varphi$ has exactly $d$ lifts, and $G$ acts on them transitively.

Proof. We consider the decomposition $\varphi^{\prime}: \operatorname{Spec} k((t)) \rightarrow \operatorname{Spec} k[[t]] \stackrel{\varphi}{\rightarrow} X$. First, we see that there are exactly $d$ lifts $\operatorname{Spec} k\left(\left(t^{1 / d}\right)\right) \rightarrow \bar{X}$ of $\varphi^{\prime}$. Let Spec $L$ be the fiber product of $q: \bar{X} \rightarrow X$ and $\varphi^{\prime}: \operatorname{Spec} k((t)) \rightarrow X$. Since $q: \bar{X} \rightarrow X$ is étale at the image of the generic point of $\varphi$, the extension $L / k((t))$ is étale. Furthermore, we have $L^{G}=k((t))$. Note that $k\left(\left(t^{1 / d^{\prime}}\right)\right)$ is the unique finite field extension of $k((t))$ of degree $d^{\prime}$ (cf. [Kol07, Theorem 1.94]). Hence $L \simeq \prod_{i=1}^{c} k\left(\left(t^{1 / a_{i}}\right)\right)$ for some $a_{i}$ and $c$. Note that if $a_{i} \neq a_{j}$, then $k\left(\left(t^{1 / a_{i}}\right)\right)$ is not isomorphic to $k\left(\left(t^{1 / a_{j}}\right)\right)$. This implies that $L^{G}$ is not a field if $a_{i} \neq a_{j}$ for some $i$ and $j$. Therefore, $L$ is decomposed as the product of $c$ copies of $k\left(\left(t^{1 / a}\right)\right)$ for some $a$ and $c$ with $a c=d$. Hence we have $\# \operatorname{Hom}_{k((t))}\left(L, k\left(\left(t^{1 / d}\right)\right)\right)=d$, and $G$ acts on $\operatorname{Hom}_{k((t))}\left(L, k\left(\left(t^{1 / d}\right)\right)\right)$ transitively:

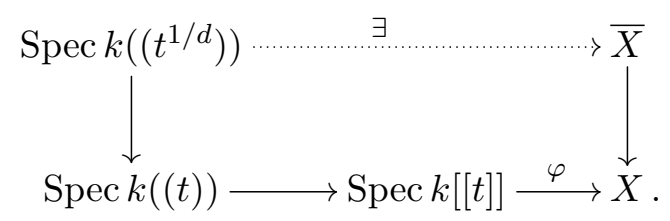

By the valuative criterion of properness on $\bar{X} \rightarrow X$, each Spec $k\left(\left(t^{1 / d}\right)\right) \rightarrow \bar{X}$ factors through Spec $k\left[\left[t^{1 / d}\right]\right] \rightarrow \bar{X}$. This completes the proof.

We have two group actions on $\bar{X}_{\infty}^{1 / d}$ :

- Let $\xi=\xi_{d} \in k$ be a $d$ th primitive root of unity in $k$. The group $\mathbb{Z} / d \mathbb{Z}=\langle\xi\rangle$ acts on $\bar{X}_{\infty}^{1 / d}$ as follows. For $\bar{\varphi}=\bar{\varphi}\left(t^{1 / d}\right) \in \bar{X}_{\infty}^{1 / d}$, we define $\bar{\varphi}\left(\xi t^{1 / d}\right)$ by the composition Spec $k\left[\left[t^{1 / d}\right]\right] \rightarrow$ Spec $k\left[\left[t^{1 / d}\right]\right] \stackrel{\bar{\varphi}}{\rightarrow} \bar{X}$, where the first map Spec $k\left[\left[t^{1 / d}\right]\right] \rightarrow \operatorname{Spec} k\left[\left[t^{1 / d}\right]\right]$ is a map induced by the ring homomorphism $k\left[\left[t^{1 / d}\right]\right] \rightarrow k\left[\left[t^{1 / d}\right]\right], t^{1 / d} \mapsto \xi t^{1 / d}$. 


\section{INVERSION OF ADJUNCTION FOR QUOTIENT SINGULARITIES}

- The group $G$ acts on $\bar{X}_{\infty}^{1 / d}$ as follows. For $\gamma \in G$ and $\bar{\varphi} \in \bar{X}_{\infty}^{1 / d}$, we define $\gamma \bar{\varphi}$ to be the composition Spec $k\left[\left[t^{1 / d}\right]\right] \stackrel{\bar{\varphi}}{\rightarrow} \bar{X} \stackrel{\gamma}{\rightarrow} \bar{X}$.

Lemma 3.2. Let $\varphi \in X_{\infty}^{\mathrm{g}}$, and let $\bar{\varphi} \in \bar{X}_{\infty}^{1 / d}$ be its lift. Then the following hold:

(1) There exists a unique $\gamma \in G$ such that $\bar{\varphi}\left(\xi t^{1 / d}\right)=\gamma \bar{\varphi}\left(t^{1 / d}\right)$.

(2) If $\bar{\varphi}^{\prime}$ is another lift and $\gamma^{\prime}$ satisfies $\bar{\varphi}^{\prime}\left(\xi t^{1 / d}\right)=\gamma^{\prime} \bar{\varphi}^{\prime}\left(t^{1 / d}\right)$, then $\gamma$ and $\gamma^{\prime}$ are in the same conjugacy class.

Proof. Since $\bar{\varphi}, \bar{\varphi}^{\prime}$ and $\bar{\varphi}\left(\xi t^{1 / d}\right)$ are lifts of $\varphi$, assertions (1) and (2) follow from Lemma 3.1.

For $\gamma \in G$, we define $\bar{X}_{\infty}^{1 / d,(\gamma)}$ and $X_{\infty}^{\mathrm{g},(\gamma)}$ as follows:

- $\bar{X}_{\infty}^{1 / d,(\gamma)}:=\left\{\bar{\varphi} \in \bar{X}_{\infty}^{1 / d} \mid \bar{\varphi}\left(\xi t^{1 / d}\right)=\gamma \bar{\varphi}\left(t^{1 / d}\right)\right\}$.

- $X_{\infty}^{\mathrm{g},(\gamma)}:=\left\{\varphi \in X_{\infty}^{\mathrm{g}} \mid \varphi\right.$ lifts to an $\operatorname{arc}$ in $\left.\bar{X}_{\infty}^{1 / d,(\gamma)}\right\}$.

LEMma 3.3. (1) We have $X_{\infty}^{\mathrm{g},(\gamma)}=X_{\infty}^{\mathrm{g},\left(\gamma^{\prime}\right)}$ if $\gamma$ and $\gamma^{\prime}$ are in the same conjugacy class.

(2) There is a natural map $\rho_{\gamma}: \bar{X}_{\infty}^{1 / d,(\gamma)} \rightarrow X_{\infty}$.

(3) The space $\bar{X}_{\infty}^{1 / d,(\gamma)}$ and $\rho_{\gamma}$ are $C_{\gamma}$-invariant, where $C_{\gamma}$ is the centralizer of $\gamma$.

(4) The $C_{\gamma}$-action on each fiber over $X_{\infty}^{\mathrm{g},(\gamma)}$ of $\rho_{\gamma}$ is transitive.

Proof. (1) If $\gamma^{\prime}=\beta \gamma \beta^{-1}$ for some $\beta \in G$ and $\bar{\varphi}\left(\xi t^{1 / d}\right)=\gamma \bar{\varphi}\left(t^{1 / d}\right)$, then $\bar{\varphi}^{\prime}:=\beta \bar{\varphi}$ satisfies $\gamma^{\prime} \bar{\varphi}^{\prime}\left(t^{1 / d}\right)=\bar{\varphi}^{\prime}\left(\xi t^{1 / d}\right)$.

(2) We may assume that $\bar{X}$ is an affine variety $\operatorname{Spec} R$. Let $\bar{\varphi} \in \bar{X}_{\infty}^{1 / d,(\gamma)}$, and let $\bar{\varphi}^{*}: R \rightarrow$ $k\left[\left[t^{1 / d}\right]\right]$ be the corresponding ring homomorphism. Suppose $a \in R^{G}$. It is sufficient to show that $\bar{\varphi}^{*}(a) \in k[[t]]$. Since $a=\gamma \cdot a$, we have

$$
\left(\bar{\varphi}^{*}(a)\right)\left(t^{1 / d}\right)=\left(\bar{\varphi}^{*}(\gamma \cdot a)\right)\left(t^{1 / d}\right)=\left(\bar{\varphi}^{*}(a)\right)\left(\xi t^{1 / d}\right),
$$

which shows that $\bar{\varphi}^{*}(a) \in k[[t]]$.

The proof of assertion (3) is straightforward.

(4) Suppose that $\bar{\varphi}_{1}, \bar{\varphi}_{2} \in \bar{X}_{\infty}^{1 / d,(\gamma)}$ are lifts of $\varphi \in X_{\infty}^{\mathrm{g},(\gamma)}$. Then by Lemma 3.1, we have $\bar{\varphi}_{1}=$ $\alpha \bar{\varphi}_{2}$ for some $\alpha \in G$. Since $\bar{\varphi}_{i}\left(\xi t^{1 / d}\right)=\gamma \bar{\varphi}_{i}\left(t^{1 / d}\right)$ holds for each $i$, it follows that $\alpha \gamma=\gamma \alpha$.

Proposition 3.4 ([DL02, Section 2.1], cf. [Yas16, Section 3]).

(1) We have $X_{\infty}^{\mathrm{g}}=\bigsqcup_{\langle\gamma\rangle \in \operatorname{Conj}(G)} X_{\infty}^{\mathrm{g},(\gamma)}$.

(2) The map $\rho_{\gamma}$ induces two maps

$$
\begin{aligned}
& \rho: \bigsqcup_{\langle\gamma\rangle \in \operatorname{Conj}(G)} \bar{X}_{\infty}^{1 / d,(\gamma)} \rightarrow X_{\infty}, \\
& \rho^{\prime}: \bigsqcup_{\langle\gamma\rangle \in \operatorname{Conj}(G)}\left(\bar{X}_{\infty}^{1 / d,(\gamma)} / C_{\gamma}\right) \rightarrow X_{\infty},
\end{aligned}
$$

and $\rho^{\prime}$ is bijective over $X_{\infty}^{\mathrm{g}}$.

Proof. Assertion (1) follows from Lemmas 3.1, 3.2(2) and 3.3(1). Assertion (2) follows from Lemma 3.3. 


\section{Y. NAKAmura AND K. Shibata}

3.2. Arc spaces of quotient singularities. Let $d$ be a positive integer, and let $\xi$ be a primitive $d$ th root of unity in $k$. Let $G \subset \mathrm{GL}_{N}(k)$ be a finite group with order $d$ which acts on $\bar{A}=\mathbb{A}_{k}^{N}=$ Spec $k\left[x_{1}, \ldots, x_{N}\right]$. Set $A=\bar{A} / G=\operatorname{Spec} k\left[x_{1}, \ldots, x_{n}\right]^{G}$. Suppose that an element $\gamma \in G$ is the diagonal matrix with entries $\xi^{e_{1}}, \ldots, \xi^{e_{n}}$ (for $0 \leqslant e_{i} \leqslant d-1$ ).

Lemma 3.5 ([DL02, Section 2.3]). The $k[t]$-ring homomorphism

$$
\epsilon_{\gamma}^{*}: k[t]\left[x_{1}, \ldots, x_{n}\right] \rightarrow k\left[t^{1 / d}\right]\left[x_{1}, \ldots, x_{n}\right], \quad x_{i} \mapsto t^{e_{i} / d} x_{i}
$$

induces a bijective map $\epsilon_{\gamma}: \bar{A}_{\infty} \rightarrow \bar{A}_{\infty}^{1 / d,(\gamma)}$.

Proof. First, we shall see that $\epsilon_{\gamma}^{*}$ induces an injective map $\epsilon_{\gamma}: \bar{A}_{\infty} \rightarrow \bar{A}_{\infty}^{1 / d}$. Let $\bar{\varphi} \in \bar{A}_{\infty}$, and let $\bar{\varphi}^{*}: k[t]\left[x_{1}, \ldots, x_{n}\right] \rightarrow k[[t]]$ be the corresponding $k[t]$-ring homomorphism. Then we define $\epsilon_{\gamma}(\bar{\varphi}) \in \bar{A}_{\infty}^{1 / d}$ to be the arc corresponding to the $k\left[t^{1 / d}\right]$-ring homomorphism

$$
k\left[t^{1 / d}\right]\left[x_{1}, \ldots, x_{n}\right] \rightarrow k\left[\left[t^{1 / d}\right]\right], \quad x_{i} \mapsto t^{e_{i} / d} \bar{\varphi}^{*}\left(x_{i}\right) .
$$

Since the map $\epsilon_{\gamma}: \bar{A}_{\infty} \rightarrow \bar{A}_{\infty}^{1 / d}$ is injective, it is sufficient to show that its image is $\bar{A}_{\infty}^{1 / d,(\gamma)}$.

Let $\bar{\varphi} \in \bar{A}_{\infty}^{1 / d}$, and let $\bar{\varphi}^{*}: k\left[t^{1 / d}\right]\left[x_{1}, \ldots, x_{n}\right] \rightarrow k\left[\left[t^{1 / d}\right]\right]$ be the corresponding $k\left[t^{1 / d}\right]$-ring homomorphism. Set $f_{i}:=\bar{\varphi}^{*}\left(x_{i}\right) \in k\left[\left[t^{1 / d}\right]\right]$. Then the condition $\bar{\varphi} \in \bar{A}_{\infty}^{1 / d,(\gamma)}$ is equivalent to the condition that

$$
\bar{\varphi}^{*}\left(x_{i}\right)\left(\xi t^{1 / d}\right)=\bar{\varphi}^{*}\left(\gamma \cdot x_{i}\right)\left(t^{1 / d}\right)
$$

holds for each $i$. This condition is equivalent to $f_{i}\left(t^{1 / d}\right) \in t^{e_{i} / d} k[[t]]$ since we have

- $\bar{\varphi}^{*}\left(x_{i}\right)\left(\xi t^{1 / d}\right)=f_{i}\left(\xi t^{1 / d}\right)$ and

- $\bar{\varphi}^{*}\left(\gamma \cdot x_{i}\right)\left(t^{1 / d}\right)=\bar{\varphi}^{*}\left(\xi^{e_{i}} x_{i}\right)\left(t^{1 / d}\right)=\xi^{e_{i}} \bar{\varphi}^{*}\left(x_{i}\right)\left(t^{1 / d}\right)=\xi^{e_{i}} f_{i}\left(t^{1 / d}\right)$.

This equivalence shows that the image of $\epsilon_{\gamma}$ is $\bar{A}_{\infty}^{1 / d,(\gamma)}$.

Lemma 3.6. (1) The map $\epsilon_{\gamma}$ is $G$-equivariant.

(2) There is a natural inclusion $\bar{A}_{\infty} / C_{\gamma} \hookrightarrow\left(\bar{A} / C_{\gamma}\right)_{\infty}$.

Proof. Assertion (1) easily follows from the definition of the $G$-actions on $\bar{A}_{\infty}$ and $\bar{A}_{\infty}^{1 / d,(\gamma)}$. Assertion (2) follows from [GW10, Proposition 12.27(2)].

Proposition 3.7 ([DL02, Section 2.7]). The map $\epsilon_{\gamma}^{*}$ induces a $k[t]$-ring homomorphism

$$
\lambda_{\gamma}^{*}: k[t]\left[x_{1}, \ldots, x_{n}\right]^{G} \rightarrow k[t]\left[x_{1}, \ldots, x_{n}\right]^{C_{\gamma}}, \quad x_{i} \mapsto t^{e_{i} / d} x_{i}
$$

and a morphism $\lambda_{\gamma}:\left(\bar{A} / C_{\gamma}\right)_{\infty} \rightarrow A_{\infty}$, and the following diagram commutes:

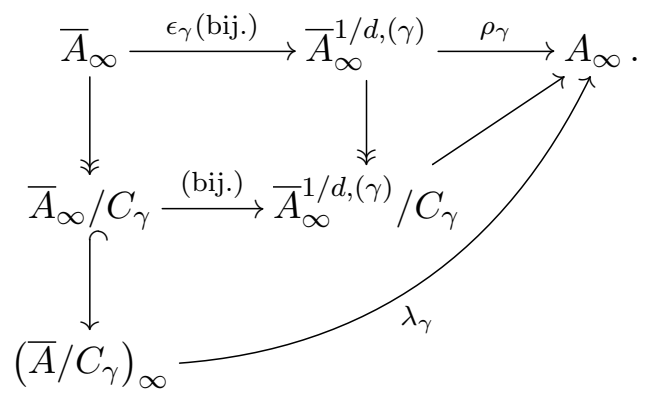

Moreover, the composite map $\bar{A}_{\infty} / C_{\gamma} \rightarrow A_{\infty}$ is bijective over $A_{\infty}^{\mathrm{g},(\gamma)}$. 


\section{INVERSION OF ADJUNCTION FOR QUOTIENT SINGULARITIES}

Proof. Note that $\epsilon_{\gamma}^{*}$ induces a $k[t]$-ring homomorphism $k[t]\left[x_{1}, \ldots, x_{n}\right]^{\langle\gamma\rangle} \rightarrow k[t]\left[x_{1}, \ldots, x_{n}\right]$, where $\langle\gamma\rangle \subset G$ is the subgroup generated by $\gamma$. Then $\lambda_{\gamma}^{*}$ is its restriction to

$$
k[t]\left[x_{1}, \ldots, x_{n}\right]^{G} \subset k[t]\left[x_{1}, \ldots, x_{n}\right]^{\langle\gamma\rangle} .
$$

The second assertion, on the bijectivity, follows from Proposition 3.4(2).

3.3. Arc spaces of quotient varieties. We keep the notation from Subsection 3.2. Suppose that $\bar{X} \subset \bar{A}$ is a $G$-invariant subvariety. In this subsection, we study the arc space of the quotient variety $X:=\bar{X} / G$.

Let $I_{X} \subset k\left[x_{1}, \ldots, x_{n}\right]^{G}$ be the defining ideal of $X$ in $A=\operatorname{Spec} k\left[x_{1}, \ldots, x_{n}\right]^{G}$. We denote by the same character $I_{X}$ the ideal of $k[t]\left[x_{1}, \ldots, x_{n}\right]^{G}$ generated by the original $I_{X}$. We denote by

$$
\widetilde{I}_{X}^{(\gamma)} \subset k[t]\left[x_{1}, \ldots, x_{n}\right]^{C_{\gamma}}, \quad \bar{I}_{X}^{(\gamma)} \subset k[t]\left[x_{1}, \ldots, x_{n}\right]
$$

the ideals generated by $\lambda_{\gamma}^{*}\left(I_{X}\right)$ and $\bar{\lambda}_{\gamma}^{*}\left(I_{X}\right)$, respectively, where we set $\bar{\lambda}_{\gamma}^{*}$ as the composition of $\lambda_{\gamma}^{*}$ and the inclusion $k[t]\left[x_{1}, \ldots, x_{n}\right]^{C_{\gamma}} \rightarrow k[t]\left[x_{1}, \ldots, x_{n}\right]$. Then we have the following commutative diagram:

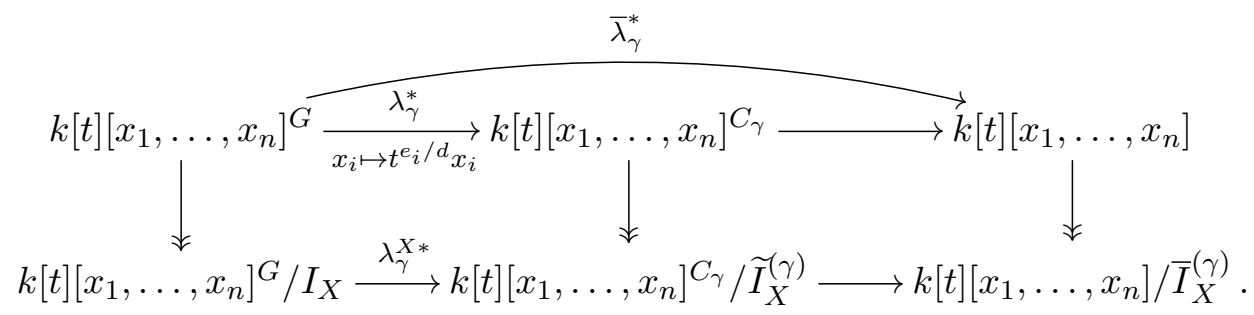

We define the arc spaces $\widetilde{X}_{\infty}^{(\gamma)}$ and $\bar{X}_{\infty}^{(\gamma)}$ as follows (see Subsection 2.4 for the definition of the arc spaces for $k[t]$-schemes):

$$
\begin{aligned}
& \widetilde{X}_{\infty}^{(\gamma)}:=\left(\operatorname{Spec} k[t]\left[x_{1}, \ldots, x_{n}\right]^{C_{\gamma}} / \widetilde{I}_{X}^{(\gamma)}\right)_{\infty}, \\
& \bar{X}_{\infty}^{(\gamma)}:=\left(\operatorname{Spec} k[t]\left[x_{1}, \ldots, x_{n}\right] / \bar{I}_{X}^{(\gamma)}\right)_{\infty} .
\end{aligned}
$$

Then we have the following diagram of arc paces:

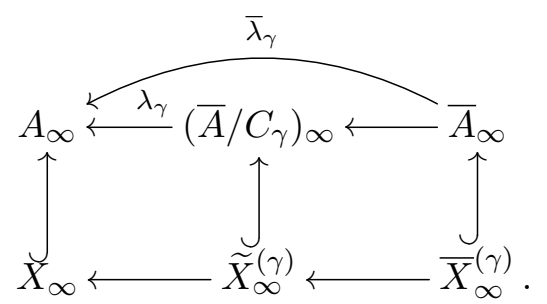

Here, the vertical arrows are closed immersions by Lemma 2.28(1). Moreover there is a natural injective map $\bar{X}_{\infty}^{(\gamma)} / C_{\gamma} \hookrightarrow \widetilde{X}_{\infty}^{(\gamma)}$ (cf. Lemma 3.6(2)).

Proposition 3.8. The ring homomorphism $\lambda_{\gamma}^{*}$ induces a morphism $\lambda_{\gamma}^{X}: \widetilde{X}_{\infty}^{(\gamma)} \rightarrow X_{\infty}$. Moreover, the composition $\bar{X}_{\infty}^{(\gamma)} / C_{\gamma} \hookrightarrow \widetilde{X}_{\infty}^{(\gamma)} \rightarrow X_{\infty}$ is bijective over $X_{\infty} \cap A_{\infty}^{\mathrm{g},(\gamma)}$.

Proof. The first assertion is straightforward.

We consider $\lambda_{\gamma}:\left(\bar{A} / C_{\gamma}\right)_{\infty} \rightarrow A_{\infty}$ and $\bar{\lambda}_{\gamma}: \bar{A}_{\infty} \rightarrow A_{\infty}$. We can identify $\bar{X}_{\infty}^{(\gamma)}, \tilde{X}_{\infty}^{(\gamma)}$ and $X_{\infty}$ with the closed subspaces of $\bar{A}_{\infty},\left(\bar{A} / C_{\gamma}\right)_{\infty}$ and $A_{\infty}$, respectively, and under these identifications, 
we have

$$
\lambda_{\gamma}^{-1}\left(X_{\infty}\right)=\widetilde{X}_{\infty}^{(\gamma)}, \quad \bar{\lambda}_{\gamma}^{-1}\left(X_{\infty}\right)=\bar{X}_{\infty}^{(\gamma)}
$$

by Lemma 2.28(2). Therefore, the second assertion follows from Proposition 3.7.

Remark 3.9. In [Yas16], Yasuda also generalizes the theory of Denef and Loeser to singular varieties. The construction in [Yas16] is intrinsic and more general, and it even works in positive characteristics. The propositions in this section are covered in the paper [Yas16]. The correspondence between the notation in this section and [Yas16] is described below.

Let $\gamma \in G$, and let $E$ be the $G$-cover of $D=\operatorname{Spec} k[[t]]$ corresponding to $\gamma$. Then $\bar{X}_{\infty}^{1 / d,(\gamma)}$ in Subsection 3.1 corresponds to the set $\operatorname{Hom}_{D}^{G}(E, V)$ of $G$-equivariant $D$-homomorphisms in [Yas16, Section 3] when $V=\bar{X} \times_{k} D$. Furthermore, $\bar{X}_{\infty}^{1 / d,(\gamma)} / C_{\gamma}$ corresponds to $J_{\infty}^{G, E} V:=$ $\operatorname{Hom}_{D}^{G}(E, V) / C_{G}(H)$ in [Yas16] when $H=\langle\gamma\rangle$ is the subgroup of $G$ generated by $\gamma$.

The diagram

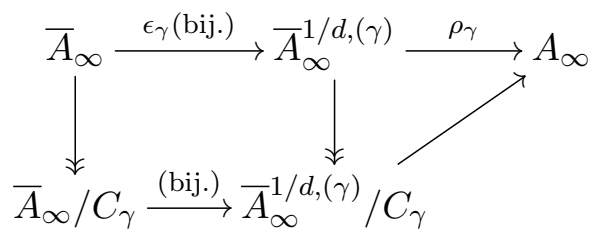

in Subsection 3.2 corresponds to the diagram

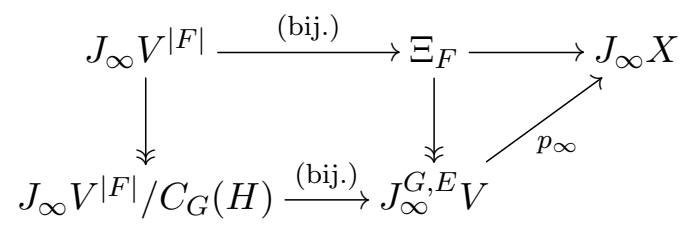

in [Yas16, Section 4] when $V=\bar{A} \times_{k} D$ and $X=V / G$.

The diagram

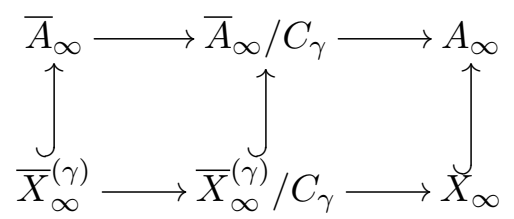

in Subsection 3.3 corresponds to

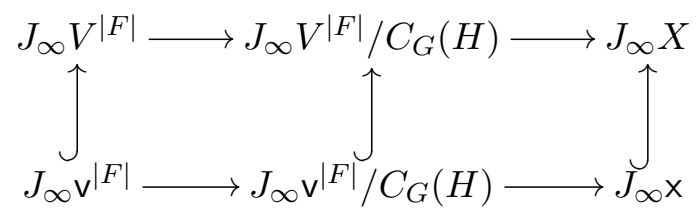

in [Yas16, Section 7] when $\mathrm{v}=\bar{X} \times_{k} D$ and $\mathrm{x}=\mathrm{v} / G$.

We note that $v^{|F|}$ in [Yas16, Section 7] corresponds to the dominant component $\bar{X}_{\text {dom }}^{(\gamma)}$ of $\bar{X}^{(\gamma)}:=\operatorname{Spec}\left(k[t]\left[x_{1}, \ldots, x_{n}\right] / \bar{I}_{X}^{(\gamma)}\right)$. In Section 4 and later, we will work on $\bar{X}^{(\gamma)}$ itself instead of $v^{|F|}=\bar{X}_{\text {dom }}^{(\gamma)}$, although their arc spaces are equal (cf. Remark 2.14(3)). One of the advantages of working on $\bar{X}^{(\gamma)}$ instead of $\bar{X}_{\text {dom }}^{(\gamma)}$ is that Lemma 2.34 can be applied to $\bar{X}^{(\gamma)}$ in the proof of Theorem 5.1. 


\section{INVERSION OF ADJUNCTION FOR QUOTIENT SINGULARITIES}

\section{Arc spaces of hyperquotient singularities}

In this section, we investigate the minimal log discrepancies of hyperquotient singularities in terms of the arc spaces of $k[t]$-schemes (Theorem 4.8).

4.1. Arc spaces of quotient singularities. In this subsection, we study the arc spaces of quotient singularities.

Let $d$ be a positive integer, and let $\xi$ be a primitive $d$ th root of unity in $k$. Let $G \subset \mathrm{GL}_{N}(k)$ be a finite group with order $d$ which acts on $\bar{A}=\mathbb{A}_{k}^{N}=\operatorname{Spec} k\left[x_{1}, \ldots, x_{N}\right]$. We denote by

$$
A:=\bar{A} / G
$$

the quotient variety. Let $Z \subset A$ be the minimal closed subset such that $\bar{A} \rightarrow A$ is étale outside $Z$. We assume $\operatorname{codim} Z \geqslant 2$, and hence the quotient map $\bar{A} \rightarrow A$ is étale in codimension one. We fix a positive integer $r$ such that $\omega_{A}^{[r]}$ is invertible (cf. [Kol13, Section 2.40]).

Let $\gamma \in G$, and let $C_{\gamma}$ be the centralizer of $\gamma$ in $G$. We denote by

$$
A^{(\gamma)}:=\bar{A} / C_{\gamma}
$$

the quotient varieties. Since $G$ is a finite group, $\gamma$ can be diagonalized with entries $\xi^{e_{1}}, \ldots, \xi^{e_{N}}$ (for $0 \leqslant e_{i} \leqslant d-1$ ) for a suitable basis $x_{1}, \ldots, x_{N}$. We set age $(\gamma)=(1 / d) \sum_{i=1}^{N} e_{i}$ (cf. [Kol13, Definition 3.20]).

Let

$$
\lambda_{\gamma}^{*}: k[t]\left[x_{1}, \ldots, x_{N}\right]^{G} \rightarrow k[t]\left[x_{1}, \ldots, x_{N}\right]^{C_{\gamma}}, \quad x_{i} \mapsto t^{e_{i} / d} x_{i}
$$

be the $k[t]$-morphism as in Section 3. We have maps

$$
\begin{aligned}
& \bar{\lambda}_{\gamma}^{*}
\end{aligned}
$$

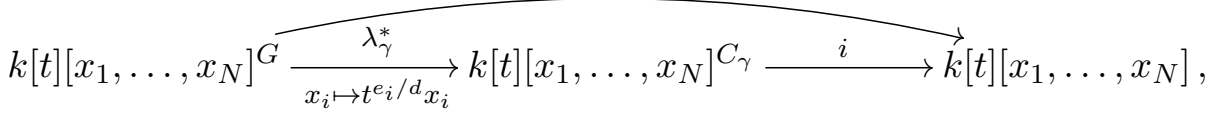

where $i$ is the inclusion map and $\bar{\lambda}_{\gamma}^{*}=i \circ \lambda_{\gamma}^{*}$ is the composite map. We have the following morphisms between the corresponding $k[t]$-varieties:

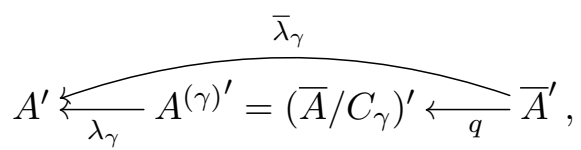

where we write $Y^{\prime}:=Y \times_{\operatorname{Spec} k} \operatorname{Spec} k[t]$ for the base change of a $k$-variety $Y$.

Lemma 4.1 (cf. [Yas16, Lemma 6.5]). Let $\alpha \in \bar{A}_{\infty}$ be an arc. Set $\alpha^{\prime}:=\left(\lambda_{\gamma} \circ q\right)_{\infty}(\alpha)$. Then it follows that

$$
\operatorname{ord}_{\alpha}\left(\operatorname{jac}_{\bar{\lambda}_{\gamma}}\right)=\frac{1}{r} \operatorname{ord}_{\alpha^{\prime}}\left(\mathfrak{n}_{r, A}\right)+\operatorname{age}(\gamma) .
$$

Proof. This follows from [Yas16, Lemma 6.5] (cf. Remark 3.9). We note that $\mathbf{v}_{V}(E)$ in $[$ Yas16, Lemma 6.5] is equal to age $(\gamma)$ when $E$ is the $G$-cover of Spec $k[[t]]$ corresponding to the conjugacy class of $\gamma$ (cf. [WY15, Lemma 4.3]).

For the reader's convenience, we give a proof in our setting below.

Let

$$
C=\operatorname{Spec} k\left[t^{1 / d}\right]\left[x_{1}, \ldots, x_{N}\right]^{G}, \quad \bar{C}=\operatorname{Spec} k\left[t^{1 / d}\right]\left[x_{1}, \ldots, x_{N}\right]
$$


We denote by the same character $\bar{\lambda}_{\gamma}$ the $k\left[t^{1 / d}\right]$-morphism $\bar{C} \rightarrow C$ induced by the original $\bar{\lambda}_{\gamma}: \bar{A}^{\prime} \rightarrow A^{\prime}$. Then $\bar{\lambda}_{\gamma}: \bar{C} \rightarrow C$ is decomposed as

$$
\bar{C} \stackrel{s}{\longrightarrow} \bar{C} \stackrel{t}{\longrightarrow} C,
$$

which correspond to $k\left[t^{1 / d}\right]$-ring homomorphisms

$$
k\left[t^{1 / d}\right]\left[x_{1}, \ldots, x_{N}\right] \overleftarrow{t_{t_{i} / d} x_{i} \longleftarrow x_{i}} k\left[t^{1 / d}\right]\left[x_{1}, \ldots, x_{N}\right] \longleftrightarrow k\left[t^{1 / d}\right]\left[x_{1}, \ldots, x_{N}\right]^{G} .
$$

Let $\beta: \operatorname{Spec} k\left[\left[t^{\frac{1}{d}}\right]\right] \rightarrow \bar{C}$ be the lift of $\alpha$. Then we have the diagram

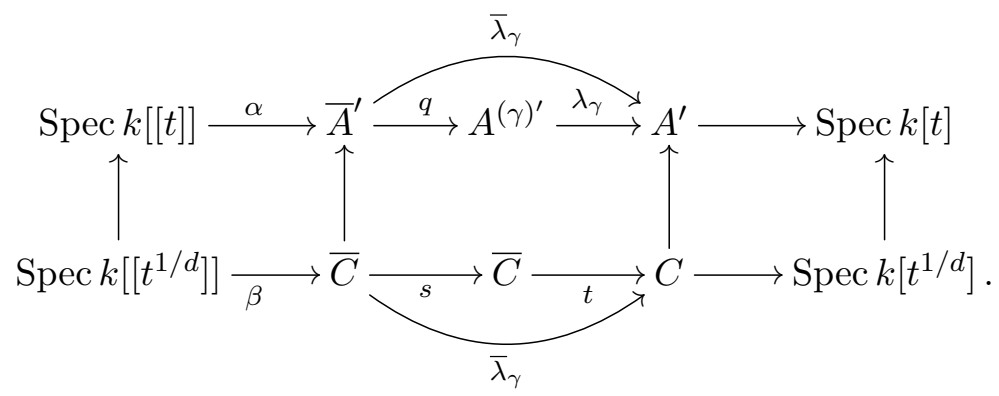

By abuse of notation, we define the order $\operatorname{ord}_{\beta}\left(\mathrm{jac}_{\bar{\lambda}_{\gamma}}\right)$ by

$$
\operatorname{ord}_{\beta}\left(\operatorname{jac}_{\bar{\lambda}_{\gamma}}\right):=\frac{1}{d} \operatorname{length}\left(\operatorname{Coker}\left(\left(\bar{\lambda}_{\gamma} \circ \beta\right)^{*} \Omega_{C / k\left[t^{1 / d}\right]} \rightarrow \beta^{*} \Omega_{\bar{C} / k\left[t^{1 / d}\right]}\right)\right),
$$

where the length is that of the cokernel considered as a $k\left[\left[t^{1 / d}\right]\right]$-module. Here, we note that $\Omega_{\bar{C} / k\left[t^{1 / d}\right]}$ is locally free because $\bar{C}$ is smooth over $k\left[t^{1 / d}\right]$. Since

$$
\begin{aligned}
& \operatorname{Coker}\left(\left(\bar{\lambda}_{\gamma} \circ \beta\right)^{*} \Omega_{C / k\left[t^{1 / d}\right]} \rightarrow \beta^{*} \Omega_{\bar{C} / k\left[t^{1 / d}\right]}\right) \\
& \quad \simeq \operatorname{Coker}\left(\left(\bar{\lambda}_{\gamma} \circ \alpha\right)^{*} \Omega_{A^{\prime} / k[t]} \rightarrow \alpha^{*} \Omega_{\bar{A}^{\prime} / k[t]}\right) \otimes_{k[[t]]} k\left[\left[t^{1 / d}\right]\right],
\end{aligned}
$$

we have $\operatorname{ord}_{\alpha}\left(\operatorname{jac}_{\bar{\lambda}_{\gamma}}\right)=\operatorname{ord}_{\beta}\left(\operatorname{jac}_{\bar{\lambda}_{\gamma}}\right)$. We also define $\operatorname{ord}_{\beta}\left(\operatorname{jac}_{s}\right)$ and $\operatorname{ord}_{\beta^{\prime}}\left(\operatorname{jac}_{t}\right)$ for $\beta^{\prime}:=s_{\infty}(\beta)$ in the same way. Then we have

$$
\operatorname{ord}_{\beta}\left(\operatorname{jac}_{\bar{\lambda}_{\gamma}}\right)=\operatorname{ord}_{\beta}\left(\operatorname{jac}_{s}\right)+\operatorname{ord}_{\beta^{\prime}}\left(\operatorname{jac}_{t}\right), \quad \operatorname{ord}_{\beta}\left(\operatorname{jac}_{s}\right)=\operatorname{age}(\gamma)
$$

by Lemma 2.10 and an easy calculation. It is then sufficient to show that we have $\operatorname{ord}_{\beta^{\prime}}\left(\mathrm{jac}_{t}\right)$ $=(1 / r) \operatorname{ord}_{\alpha^{\prime}}\left(\mathfrak{n}_{r, A}\right)$.

In the commutative diagram

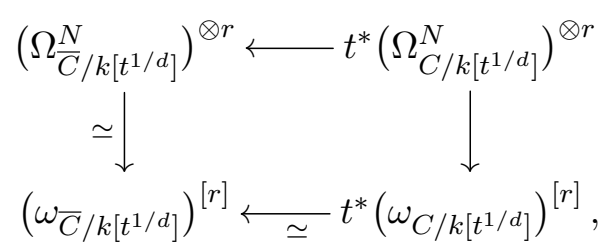

the vertical map $\left(\Omega_{\bar{C} / k\left[t^{1 / d}\right]}^{N}\right)^{\otimes r} \rightarrow\left(\omega_{\bar{C} / k\left[t^{1 / d}\right]}\right)^{[r]}$ is an isomorphism because $\bar{C}$ is smooth over $k\left[t^{1 / d}\right]$. Furthermore, $t^{*}\left(\omega_{C / k\left[t^{1 / d}\right]}\right)^{[r]} \rightarrow\left(\omega_{\bar{C} / k\left[t^{1 / d}\right]}\right)^{[r]}$ is an isomorphism since $t$ is étale in codimension one. By combining this with Lemma 2.13(2), we conclude that $r \operatorname{ord}_{\beta^{\prime}}\left(\mathrm{jac}_{t}\right)=$ $\operatorname{ord}_{\alpha^{\prime}}\left(\mathfrak{n}_{r, A}\right)$. This completes the proof. 


\section{INVERSION OF ADJUNCTION FOR QUOTIENT SINGULARITIES}

4.2. Arc spaces of hyperquotient singularities. In this subsection, we study the arc spaces of hyperquotient singularities.

Let $\xi, G, \gamma, C_{\gamma}, \bar{A}, A, Z, A^{(\gamma)}, r, \lambda_{\gamma}^{*}, i, \bar{\lambda}_{\gamma}^{*}, \lambda_{\gamma}, q, \bar{\lambda}_{\gamma}$ be as in Subsection 4.1. Let $f_{1}, \ldots, f_{c} \in$ $k\left[x_{1}, \ldots, x_{N}\right]^{G}$ be a regular sequence which is contained in the maximal ideal at the origin. We set

$$
B:=\operatorname{Spec} k\left[x_{1}, \ldots, x_{N}\right]^{G} /\left(f_{1}, \ldots, f_{c}\right), \quad \bar{B}:=\operatorname{Spec} k\left[x_{1}, \ldots, x_{N}\right] /\left(f_{1}, \ldots, f_{c}\right) .
$$

Suppose that $B$ is normal. Note that $\omega_{B}^{[r]}$ is invertible since $\omega_{A}^{[r]}$ is invertible. We define ideals $I$, $\widetilde{I}^{(\gamma)}$ and $\bar{I}^{(\gamma)}$ as

$$
\begin{aligned}
I & :=\left(f_{1}, \ldots, f_{c}\right) \subset k[t]\left[x_{1}, \ldots, x_{N}\right]^{G}, \\
\widetilde{I}^{(\gamma)} & :=\left(\lambda_{\gamma}^{*}\left(f_{1}\right), \ldots, \lambda_{\gamma}^{*}\left(f_{c}\right)\right) \subset k[t]\left[x_{1}, \ldots, x_{N}\right]^{C_{\gamma}}, \\
\bar{I}^{(\gamma)} & :=\left(\bar{\lambda}_{\gamma}^{*}\left(f_{1}\right), \ldots, \bar{\lambda}_{\gamma}^{*}\left(f_{c}\right)\right) \subset k[t]\left[x_{1}, \ldots, x_{N}\right] .
\end{aligned}
$$

We define $k[t]$-schemes $B^{\prime}, \widetilde{B}^{(\gamma)}$ and $\bar{B}^{(\gamma)}$ as

$$
\begin{aligned}
B^{\prime} & =\operatorname{Spec} k[t]\left[x_{1}, \ldots, x_{N}\right]^{G} / I, \\
\widetilde{B}^{(\gamma)} & =\operatorname{Spec} k[t]\left[x_{1}, \ldots, x_{N}\right]^{C_{\gamma}} / \widetilde{I}^{(\gamma)}, \\
\bar{B}^{(\gamma)} & =\operatorname{Spec} k[t]\left[x_{1}, \ldots, x_{N}\right] / \bar{I}^{(\gamma)} .
\end{aligned}
$$

Then we have the following diagram with the induced $k[t]$-morphisms $\mu_{\gamma}^{*}, \bar{\mu}_{\gamma}^{*}$ and $j$ :

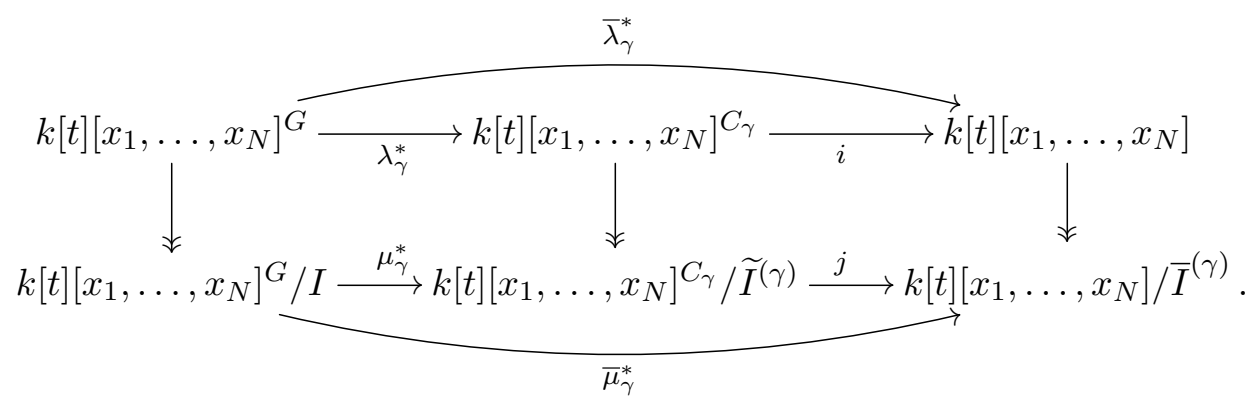

Furthermore, we have the following morphisms between the corresponding $k[t]$-schemes:

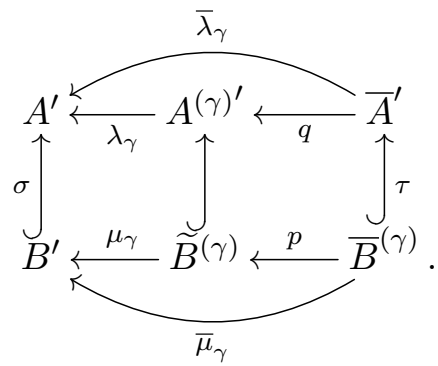

First we give an easy observation on the intersection of $B$ and $Z$.

Lemma 4.2. We have $\operatorname{codim}_{B}(Z \cap B) \geqslant 2$. In particular, $\bar{B} \rightarrow B$ is étale in codimension one, and $\bar{B}$ is normal.

Proof. Since $\operatorname{codim}_{A}(Z) \geqslant 2$, we have $A_{\text {sing }}=Z$ by the purity of the branch locus (cf. [Nag59]). Since $f_{1}, \ldots, f_{c} \in A$ is a regular sequence, it follows that $Z \cap B \subset B_{\text {sing }}$ (cf. [Sta21, tag 00NU]). By the normality of $B$, we have $\operatorname{codim}_{B}(Z \cap B) \geqslant 2$. Therefore, $\bar{B} \rightarrow B$ is étale in codimension one. 
Furthermore, we have $\operatorname{codim}_{\bar{B}}\left(\bar{B}_{\text {sing }}\right) \geqslant 2$. Since $\bar{B}$ is Cohen-Macaulay, the normality follows from Serre's criterion.

Remark 4.3. Let $\bar{B}_{t \neq 0}^{(\gamma)}$ be the open subscheme of $\bar{B}^{(\gamma)}$ defined by $t \neq 0$. We shall see in this remark that we have a surjective étale morphism

$$
\left(\mathbb{A}^{1} \backslash\{0\}\right) \times \bar{B} \rightarrow \bar{B}_{t \neq 0}^{(\gamma)} .
$$

First, we have the following natural morphisms:

$$
\begin{aligned}
& \bar{B}^{(\gamma)}=\operatorname{Spec}\left(k[t]\left[x_{1}, \ldots, x_{N}\right] /\left(\bar{\lambda}_{\gamma}^{*}\left(f_{1}\right), \ldots, \bar{\lambda}_{\gamma}^{*}\left(f_{c}\right)\right)\right) \\
& \uparrow \\
& \bar{B}_{t \neq 0}^{(\gamma)}:=\operatorname{Spec}\left(k\left[t, t^{-1}\right]\left[x_{1}, \ldots, x_{N}\right] /\left(\bar{\lambda}_{\gamma}^{*}\left(f_{1}\right), \ldots, \bar{\lambda}_{\gamma}^{*}\left(f_{c}\right)\right)\right) \\
& \text { 个étale } \\
& \operatorname{Spec}\left(k\left[t^{1 / d}, t^{-1 / d}\right]\left[x_{1}, \ldots, x_{N}\right] /\left(\bar{\lambda}_{\gamma}^{*}\left(f_{1}\right), \ldots, \bar{\lambda}_{\gamma}^{*}\left(f_{c}\right)\right)\right) \\
& \downarrow \\
& \left(\mathbb{A}^{1} \backslash\{0\}\right) \times \bar{B} \simeq \operatorname{Spec}\left(k\left[t^{1 / d}, t^{-1 / d}\right]\left[x_{1}, \ldots, x_{N}\right] /\left(f_{1}, \ldots, f_{c}\right)\right) .
\end{aligned}
$$

Here the second morphism is étale since it is induced by the étale homomorphism $k\left[t, t^{-1}\right] \rightarrow$ $k\left[t^{1 / d}, t^{-1 / d}\right]$. The third morphism, which is actually an isomorphism, is induced by the ring isomorphism

$$
k\left[t^{1 / d}, t^{-1 / d}\right]\left[x_{1}, \ldots, x_{N}\right] \rightarrow k\left[t^{1 / d}, t^{-1 / d}\right]\left[x_{1}, \ldots, x_{N}\right], \quad x_{i} \mapsto t^{e_{i} / d} x_{i},
$$

whose inverse map is $x_{i} \mapsto t^{-e_{i} / d} x_{i}$. Therefore, we have a surjective étale morphism

$$
\left(\mathbb{A}^{1} \backslash\{0\}\right) \times \bar{B} \rightarrow \bar{B}_{t \neq 0}^{(\gamma)} .
$$

By Remark 4.3, the $k[t]$-scheme $\bar{B}^{(\gamma)}$ satisfies the condition $(\star \star)_{n}$ for $n:=N-c$. Furthermore, $B^{\prime}$ and $\widetilde{B}^{(\gamma)}$ also satisfy the condition $(\star \star)_{n}$.

Remark 4.4. Note that $\bar{B}^{(\gamma)}$ is not a complete intersection in $\bar{A}^{\prime}$ in general because $\bar{\lambda}_{\gamma}^{*}\left(f_{1}\right), \ldots$, $\bar{\lambda}_{\gamma}^{*}\left(f_{c}\right)$ is not necessarily a regular sequence. In fact, $\bar{B}^{(\gamma)}$ is not pure-dimensional in general (cf. Example 2.6). Hence we do not have the standard definition of the canonical sheaf on $\bar{B}^{(\gamma)}$ (cf. Definition 2.11).

We define the following invertible sheaf $L_{\bar{B}^{(\gamma)}}$ on $\bar{B}^{(\gamma)}$ instead:

$$
L_{\bar{B}^{(\gamma)}}:=\bar{\mu}_{\gamma}^{*}\left(\operatorname{det}^{-1}\left(I / I^{2}\right)\right) \otimes_{\mathcal{O}_{\bar{B}}(\gamma)} \tau^{*} \omega_{\bar{A}^{\prime} / k[t]} .
$$

Here $\operatorname{det}^{-1}\left(I / I^{2}\right)=\left(\bigwedge^{c}\left(I / I^{2}\right)\right)^{*}$ is an invertible sheaf on $B^{\prime}$. Then $L_{\bar{B}^{(\gamma)}}$ fills the role of the canonical sheaf as follows.

LEMma 4.5. There is a canonical morphism $\eta: \Omega_{\bar{B}^{(\gamma)} / k[t]}^{n} \rightarrow L_{\bar{B}^{(\gamma)}}$ with the following conditions:

(1) We have $\operatorname{Im}(\eta)=\operatorname{Jac}_{\bar{B}^{(\gamma)} / k[t]} \otimes L_{\bar{B}^{(\gamma)}}$ 


\section{INVERSION OF ADJUNCTION FOR QUOTIENT SINGULARITIES}

(2) There exists a commutative diagram

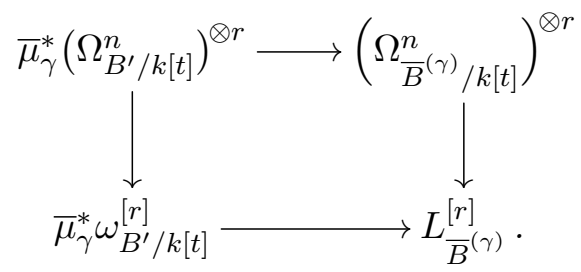

Proof. First, we shall see that there exists a canonical morphism

$$
\bar{\mu}_{\gamma}^{*}\left(\operatorname{det}\left(I / I^{2}\right)\right) \otimes_{\mathcal{O}_{\bar{B}}(\gamma)} \Omega_{\bar{B}^{(\gamma)} / k[t]}^{n} \rightarrow \tau^{*} \omega_{\bar{A}^{\prime} / k[t]},
$$

whose image is $\operatorname{Jac}_{\bar{B}^{(\gamma)} / k[t]} \otimes \tau^{*} \omega_{\bar{A}^{\prime} / k[t]}$. We set

$$
R:=k[t]\left[x_{1}, \ldots, x_{N}\right], \quad \bar{S}:=k[t]\left[x_{1}, \ldots, x_{N}\right] / \bar{I}^{(\gamma)}, \quad S:=k[t]\left[x_{1}, \ldots, x_{N}\right]^{G} / I .
$$

Let $N \subset \Omega_{R / k[t]}$ be the $R$-submodule which is generated by $d f$ for $f \in \bar{I}^{(\gamma)}$. Then we have $\Omega_{\bar{S} / k[t]} \simeq\left(\Omega_{R / k[t]} / N\right) \otimes_{R} \bar{S}$. Since $\bar{I}^{(\gamma)}$ is generated by $c$ elements, we have $\bigwedge^{c+1} N=0$. Hence a canonical map $\bigwedge^{c} N \otimes_{R} \bigwedge^{N-c} \Omega_{R / k[t]} \rightarrow \Omega_{R / k[t]}^{N}$ induces

$$
\bigwedge^{c} N \otimes_{R} \bigwedge^{N-c}\left(\Omega_{R / k[t]} / N\right) \rightarrow \Omega_{R / k[t]}^{N}
$$

By taking $-\otimes_{R} \bar{S}$ and composing with

$$
I / I^{2} \otimes_{S} \bar{S} \rightarrow \bar{I}^{(\gamma)} /\left(\bar{I}^{(\gamma)}\right)^{2} \stackrel{\bar{h} \mapsto d(h) \otimes 1}{\longrightarrow} N \otimes_{R} \bar{S},
$$

we have a canonical map

$$
\left(\bigwedge^{c}\left(I / I^{2}\right) \otimes_{S} \bar{S}\right) \otimes_{\bar{S}} \Omega_{\bar{S} / k[t]}^{N-c} \rightarrow \Omega_{R / k[t]}^{N} \otimes_{R} \bar{S} .
$$

The $\bar{S}$-module $\left(\bigwedge^{c}\left(I / I^{2}\right) \otimes_{S} \bar{S}\right) \otimes_{\bar{S}} \Omega_{\bar{S} / k[t]}^{N-c}$ is generated by the elements of the form

$$
\left(\bar{f}_{1} \wedge \cdots \wedge \bar{f}_{c}\right) \otimes\left(d x_{i_{1}} \wedge \cdots \wedge d x_{i_{N-c}}\right)
$$

and its image in $\Omega_{R / k[t]}^{N} \otimes_{R} \bar{S}$ is

$$
d\left(\bar{\lambda}_{\gamma}^{*}\left(f_{1}\right)\right) \wedge \cdots \wedge d\left(\bar{\lambda}_{\gamma}^{*}\left(f_{c}\right)\right) \wedge d x_{i_{1}} \wedge \cdots \wedge d x_{i_{N-c}} .
$$

We note that this is equal to $\pm \Delta \cdot d x_{1} \wedge \cdots \wedge d x_{N}$, where $\Delta$ is the determinant of the Jacobian matrix with respect to $\bar{\lambda}_{\gamma}^{*}\left(f_{i}\right)$ for $1 \leqslant i \leqslant c$ and $\partial x_{j}$ for $j \in\{1, \ldots, N\} \backslash\left\{i_{1}, \ldots i_{N-c}\right\}$. Hence we have a canonical morphism

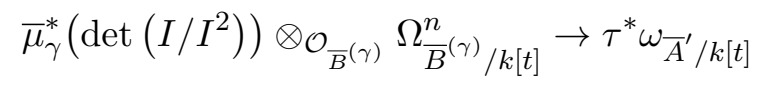

whose image is $\operatorname{Jac}_{\bar{B}^{(\gamma)} / k[t]} \otimes \tau^{*} \omega_{\bar{A}^{\prime} / k[t]}$. We have proved assertion (1).

For assertion (2), we note that a canonical map $\bar{\mu}_{\gamma}^{*} \omega_{B^{\prime} / k[t]}^{[r]} \rightarrow L_{\bar{B}^{(\gamma)}}^{[r]}$ is induced by the isomorphism $\omega_{B^{\prime} / k[t]} \simeq \operatorname{det}^{-1}\left(I / I^{2}\right) \otimes_{\mathcal{O}_{B^{\prime}}} \sigma^{*} \omega_{A^{\prime} / k[t]}$ obtained by adjunction. The commutativity of the diagram is obvious.

In the same way as in Definition 2.11, we denote by $\mathfrak{n}_{1, p}^{\prime}$ and $\mathfrak{n}_{1, \bar{\mu}_{\gamma}}^{\prime}$ the ideal sheaves on $\bar{B}^{(\gamma)}$ 


\section{Y. NAKAmura AND K. Shibata}

satisfying

$$
\operatorname{Im}\left(p^{*} \Omega_{\widetilde{B}^{(\gamma) / k[t]}}^{n} \rightarrow L_{\bar{B}^{(\gamma)}}\right)=\mathfrak{n}_{1, p}^{\prime} \otimes L_{\bar{B}^{(\gamma)}}, \quad \operatorname{Im}\left(\bar{\mu}_{\gamma}^{*} \Omega_{B^{\prime} / k[t]}^{n} \rightarrow L_{\bar{B}^{(\gamma)}}\right)=\mathfrak{n}_{1, \bar{\mu}_{\gamma}}^{\prime} \otimes L_{\bar{B}^{(\gamma)}} .
$$

We now prove Lemmas 4.6 and 4.7 on relations on orders, which will be used in the proof of Theorem 4.8.

Lemma 4.6. Let $\alpha \in \bar{B}_{\infty}^{(\gamma)}$ be an arc with $\operatorname{ord}_{\alpha}\left(\operatorname{Jac}_{\bar{B}^{(\gamma)} / k[t]}\right)<\infty$. Then the following hold:

(1) $\operatorname{ord}_{\alpha}\left(\operatorname{jac}_{p}\right)+\operatorname{ord}_{\alpha}\left(\operatorname{Jac}_{\bar{B}}^{(\gamma) / k[t]}\right)=\operatorname{ord}_{\alpha}\left(\mathfrak{n}_{1, p}^{\prime}\right)$.

(2) $\operatorname{ord}_{\alpha}\left(\operatorname{jac}_{\bar{\mu}_{\gamma}}\right)+\operatorname{ord}_{\alpha}\left(\operatorname{Jac}_{\bar{B}^{(\gamma)} / k[t]}\right)=\operatorname{ord}_{\alpha}\left(\mathfrak{n}_{1, \bar{\mu}_{\gamma}}^{\prime}\right)$.

Proof. The proof of Lemma 2.13(2) works by Lemma 4.5(1).

Lemma 4.7. Let $\alpha \in \bar{B}_{\infty}^{(\gamma)}$ be an arc. Set $\alpha^{\prime}:=\bar{\mu}_{\gamma \infty}(\alpha)$. Suppose $\alpha^{\prime} \notin Z_{\infty}$. Then it follows that

$$
\operatorname{ord}_{\alpha}\left(\mathfrak{n}_{1, \bar{\mu}_{\gamma}}^{\prime}\right)=\frac{1}{r} \operatorname{ord}_{\alpha^{\prime}}\left(\mathfrak{n}_{r, B^{\prime}}\right)+\operatorname{age}(\gamma) .
$$

Proof. We have two diagrams (cf. Lemma 4.5(2)):
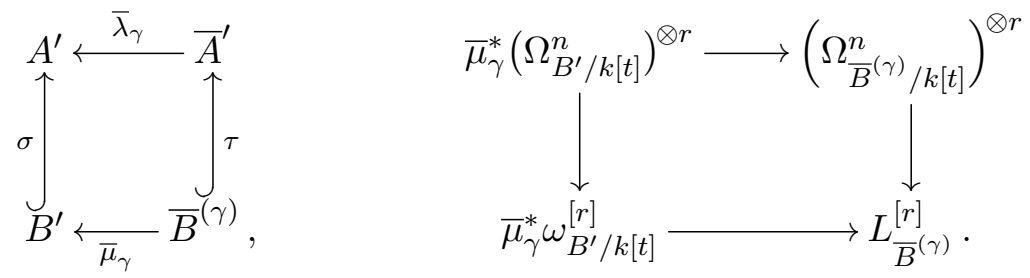

We denote by $\mathfrak{r}_{\bar{B}}^{(\gamma)}$ the ideal sheaf on $\bar{B}^{(\gamma)}$ satisfying

$$
\operatorname{Im}\left(\bar{\mu}_{\gamma}^{*} \omega_{B^{\prime} / k[t]}^{[r]} \rightarrow L_{\bar{B}^{(\gamma)}}^{[r]}\right)=\mathfrak{r}_{\bar{B}^{(\gamma)}} \otimes L_{\bar{B}^{(\gamma)}}^{[r]} .
$$

We also denote by $\mathfrak{r}_{\bar{A}}$ the ideal sheaf on $\bar{A}^{\prime}$ satisfying

$$
\operatorname{Im}\left(\bar{\lambda}_{\gamma}^{*} \omega_{A^{\prime} / k[t]}^{[r]} \rightarrow \omega_{\bar{A}^{\prime} / k[t]}^{[r]}\right)=\mathfrak{r}_{\bar{A}^{\prime}} \otimes \omega_{\bar{A}^{\prime} / k[t]}^{[r]} .
$$

By the definition of the Nash ideals (Definition 2.11), we have

$$
r \operatorname{ord}_{\alpha}\left(\mathfrak{n}_{1, \bar{\mu}_{\gamma}}^{\prime}\right)=\operatorname{ord}_{\alpha}\left(\mathfrak{r}_{\bar{B}}^{(\gamma)}\right)+\operatorname{ord}_{\alpha^{\prime}}\left(\mathfrak{n}_{r, B^{\prime}}\right) .
$$

On the other hand, by Lemma 4.1, we have

$$
\begin{aligned}
r \operatorname{age}(\gamma) & =r \operatorname{ord}_{\alpha}\left(\operatorname{jac}_{\bar{\lambda}_{\gamma}}\right)-\operatorname{ord}_{\alpha^{\prime}}\left(\mathfrak{n}_{r, A^{\prime}}\right) \\
& =r \operatorname{ord}_{\alpha}\left(\mathfrak{n}_{1, \bar{\lambda}_{\gamma}}\right)-\operatorname{ord}_{\alpha^{\prime}}\left(\mathfrak{n}_{r, A^{\prime}}\right) \\
& =\operatorname{ord}_{\alpha}\left(\mathfrak{r}_{\bar{A}^{\prime}}\right) .
\end{aligned}
$$

Here the second equality follows from Lemma 2.13(2) and the fact $\mathfrak{n}_{1, \bar{A}^{\prime}}=\mathcal{O}_{\bar{A}^{\prime}}$. The third equality follows from the definition of the ideals $\mathfrak{n}_{1, \bar{\lambda}_{\gamma}}, \mathfrak{n}_{r, A^{\prime}}$ and $\mathfrak{r}_{\bar{A}^{\prime}}$ and the fact that $\operatorname{ord}_{\alpha^{\prime}}\left(\mathfrak{n}_{r, A^{\prime}}\right)<\infty$, which follows from the assumption $\alpha^{\prime} \notin Z_{\infty}$. Hence it is sufficient to show that $\operatorname{ord}_{\alpha}\left(\mathfrak{r}_{\bar{B}^{(\gamma)}}\right)=$ $\operatorname{ord}_{\alpha}\left(\mathfrak{r}_{\bar{A}^{\prime}}\right)$.

Since

$$
\omega_{B^{\prime} / k[t]} \simeq \operatorname{det}^{-1}\left(I / I^{2}\right) \otimes_{\mathcal{O}_{B^{\prime}}} \sigma^{*} \omega_{A^{\prime} / k[t]}
$$

holds by the adjunction formula, we have $\mathfrak{r}_{\bar{B}}^{(\gamma)}=\mathfrak{r}_{\bar{A}^{\prime}} \mathcal{O}_{\bar{B}^{(\gamma)}}$ by the definition of $L_{\bar{B}^{(\gamma)}}$. This completes the proof. 


\section{INVERSION OF ADJUNCTION FOR QUOTIENT SINGULARITIES}

4.3. Minimal log discrepancies of hyperquotient singularities via jet schemes. In this subsection, we investigate the minimal log discrepancies of hyperquotient singularities in terms of arc spaces. We use the notation from Subsection 4.2.

TheOREM 4.8. Let $x=0 \in B$ be the origin, let $\mathfrak{a} \subset k\left[x_{1}, \ldots, x_{N}\right]^{G} / I$ be a non-zero ideal, and let $\delta$ be a non-negative real number. Then

$$
\operatorname{mld}_{x}\left(B, \mathfrak{a}^{\delta}\right)=\inf _{w, b_{1} \in \mathbb{Z}_{\geqslant 0}, \gamma \in G}\left\{\operatorname{codim}\left(C_{w, \gamma, b_{1}}\right)+\operatorname{age}(\gamma)-b_{1}-\delta w\right\}
$$

holds for

$$
C_{w, \gamma, b_{1}}=\operatorname{Cont}^{w}\left(\mathfrak{a} \mathcal{O}_{\bar{B}^{(\gamma)}}\right) \cap \operatorname{Cont}^{\geqslant 1}\left(\mathfrak{m}_{x} \mathcal{O}_{\bar{B}^{(\gamma)}}\right) \cap \operatorname{Cont}^{b_{1}}\left(\mathrm{Jac}_{\bar{B}^{(\gamma)} / k[t]}\right),
$$

where $\mathfrak{m}_{x} \subset \mathcal{O}_{B}$ is the maximal ideal corresponding to the closed point $x \in B$.

Proof. By [EM09, Theorem 7.4], we have

$$
\operatorname{mld}_{x}\left(B, \mathfrak{a}^{\delta}\right)=\inf _{w, m_{3} \in \mathbb{Z}_{\geqslant 0}}\left\{\operatorname{codim}\left(\operatorname{Cont}^{w}(\mathfrak{a}) \cap \operatorname{Cont}^{m_{3}}\left(\mathfrak{n}_{r, B}\right) \cap \operatorname{Cont}^{\geqslant 1}\left(\mathfrak{m}_{x}\right)\right)-\frac{m_{3}}{r}-\delta w\right\} .
$$

Note that $\mathfrak{n}_{r, B^{\prime}}=\mathfrak{n}_{r, B} \mathcal{O}_{B^{\prime}}$ holds by Remark 2.12 and $B_{m}^{\prime}=B_{m}$ holds for $m \in \mathbb{Z}_{\geqslant 0} \cup\{\infty\}$ by Remark 2.4.

Let $w, b_{1}, b_{2}, b_{3}, m_{1}, m_{2}, m_{3} \in \mathbb{Z}_{\geqslant 0}$ and $\gamma \in G$. We denote by $D_{w, \gamma, b_{1}, b_{2}, b_{3}, m_{1}, m_{2}, m_{3}} \subset \bar{B}_{\infty}^{(\gamma)}$ the cylinder

$$
\begin{aligned}
\operatorname{Cont}^{w}\left(\mathfrak{a O}_{\bar{B}^{(\gamma)}}\right) \cap \operatorname{Cont}^{\geqslant 1}\left(\mathfrak{m}_{x} \mathcal{O}_{\bar{B}^{(\gamma)}}\right) \cap \operatorname{Cont}^{b_{1}}\left(\operatorname{Jac}_{\bar{B}^{(\gamma)} / k[t]}\right) \cap \operatorname{Cont}^{b_{2}}\left(\operatorname{Jac}_{\widetilde{B}^{(\gamma)} / k[t]} \mathcal{O}_{\bar{B}}(\gamma)\right) \\
\cap \operatorname{Cont}^{b_{3}}\left(\operatorname{Jac}_{B^{\prime} / k[t]} \mathcal{O}_{\bar{B}}(\gamma)\right) \cap \operatorname{Cont}^{m_{1}}\left(\mathfrak{n}_{1, p}^{\prime}\right) \cap \operatorname{Cont}^{m_{2}}\left(\mathfrak{n}_{1, \bar{\mu}_{\gamma}}^{\prime}\right) \cap \operatorname{Cont}^{m_{3}}\left(\mathfrak{n}_{r, B^{\prime}} \mathcal{O}_{\bar{B}}(\gamma)\right) .
\end{aligned}
$$

By Propositions 3.4 and 3.8, we have

$$
\begin{aligned}
& \operatorname{Cont}^{w}(\mathfrak{a}) \cap \operatorname{Cont}^{m_{3}}\left(\mathfrak{n}_{r, B}\right) \cap \operatorname{Cont}^{\geqslant 1}\left(\mathfrak{m}_{x}\right) \backslash Z_{\infty} \\
& \quad=\bigsqcup_{\langle\gamma\rangle \in \operatorname{Conj}(G)} \mu_{\gamma \infty} \circ p_{\infty}\left(\operatorname{Cont}^{w}\left(\mathfrak{a} \mathcal{O}_{\bar{B}}^{(\gamma)}\right) \cap \operatorname{Cont}^{m_{3}}\left(\mathfrak{n}_{r, B} \mathcal{O}_{\bar{B}^{(\gamma)}}\right) \cap \operatorname{Cont}^{\geqslant 1}\left(\mathfrak{m}_{x} \mathcal{O}_{\bar{B}^{(\gamma)}}\right)\right) \backslash Z_{\infty} .
\end{aligned}
$$

Note that $\mu_{\gamma \infty} \circ p_{\infty}(C)$ is a thin set of $B_{\infty}^{\prime}$ for any thin set $C$ of $\bar{B}_{\infty}^{(\gamma)}$. Hence we have

$$
\begin{aligned}
& \operatorname{codim}\left(\operatorname{Cont}^{w}(\mathfrak{a}) \cap \operatorname{Cont}^{m_{3}}\left(\mathfrak{n}_{r, B}\right) \cap \operatorname{Cont}^{\geqslant 1}\left(\mathfrak{m}_{x}\right)\right) \\
& \quad=\min _{\gamma, b_{1}, b_{2}, b_{3}, m_{1}, m_{2}} \operatorname{codim}\left(\mu_{\gamma \infty} \circ p_{\infty}\left(D_{w, \gamma, b_{1}, b_{2}, b_{3}, m_{1}, m_{2}, m_{3}}\right)\right)
\end{aligned}
$$

by Proposition 2.25. On the other hand, again by Proposition 2.25, we have

$$
\operatorname{codim}\left(C_{w, \gamma, b_{1}}\right)=\min _{b_{2}, b_{3}, m_{1}, m_{2}, m_{3}} \operatorname{codim}\left(D_{w, \gamma, b_{1}, b_{2}, b_{3}, m_{1}, m_{2}, m_{3}}\right) .
$$

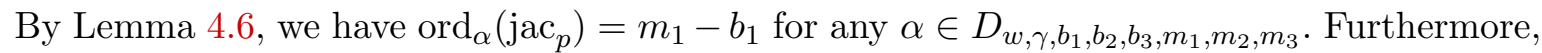
$\operatorname{ord}\left(\operatorname{Jac}_{\bar{B}^{(\gamma)} / k[t]}\right)$ and $\operatorname{ord}\left(\operatorname{Jac}_{\widetilde{B}^{(\gamma)} / k[t]}\right)$ take constant values $b_{1}$ and $b_{2}$ on $D_{w, \gamma, b_{1}, b_{2}, b_{3}, m_{1}, m_{2}, m_{3}}$ and $p_{\infty}\left(D_{w, \gamma, b_{1}, b_{2}, b_{3}, m_{1}, m_{2}, m_{3}}\right)$, respectively. Hence by applying Proposition 2.35 to $p$, we have

$$
\operatorname{codim}\left(p_{\infty}\left(D_{w, \gamma, b_{1}, b_{2}, b_{3}, m_{1}, m_{2}, m_{3}}\right)\right)=\operatorname{codim}\left(D_{w, \gamma, b_{1}, b_{2}, b_{3}, m_{1}, m_{2}, m_{3}}\right)+m_{1}-b_{1} .
$$

Note that $\operatorname{codim}_{B}(B \cap Z) \geqslant 2$ by Lemma 4.2 and that $\left.\mu_{\gamma \infty}\right|_{p_{\infty}\left(D_{w, \gamma, b_{1}, b_{2}, b_{3}, m_{1}, m_{2}, m_{3}}\right)}$ is injective outside $Z_{\infty}$ by Proposition 3.8. By Lemmas 2.10 and 4.6, we have

$$
\operatorname{ord}_{\alpha}\left(\mathrm{jac}_{\mu_{\gamma}}\right)=\left(m_{2}-b_{1}\right)-\left(m_{1}-b_{1}\right)=m_{2}-m_{1}
$$




\section{Y. NAKAmURA AND K. Shibata}

for any $\alpha \in p_{\infty}\left(D_{w, \gamma, b_{1}, b_{2}, b_{3}, m_{1}, m_{2}, m_{3}}\right)$. Furthermore, ord $\left(\operatorname{Jac}_{B^{\prime} / k[t]}\right)$ takes a constant value $b_{3}$ on $\left(\mu_{\gamma} \circ p\right)_{\infty}\left(D_{w, \gamma, b_{1}, b_{2}, b_{3}, m_{1}, m_{2}, m_{3}}\right)$. Hence by applying Proposition 2.33 to $\mu_{\gamma}$, we have

$$
\begin{aligned}
& \operatorname{codim}\left(\left(\mu_{\gamma} \circ p\right)_{\infty}\left(D_{w, \gamma, b_{1}, b_{2}, b_{3}, m_{1}, m_{2}, m_{3}}\right)\right) \\
& \quad=\operatorname{codim}\left(p_{\infty}\left(D_{w, \gamma, b_{1}, b_{2}, b_{3}, m_{1}, m_{2}, m_{3}}\right)\right)+m_{2}-m_{1} .
\end{aligned}
$$

By Lemma 4.7, we have $m_{2}-m_{3} / r=\operatorname{age}(\gamma)$ if $\left(\mu_{\gamma} \circ p\right)_{\infty}\left(D_{w, \gamma, b_{1}, b_{2}, b_{3}, m_{1}, m_{2}, m_{3}}\right) \backslash Z_{\infty}$ is non-empty. Thus we obtain

$$
\begin{aligned}
& \operatorname{codim}\left(D_{w, \gamma, b_{1}, b_{2}, b_{3}, m_{1}, m_{2}, m_{3}}\right)+\operatorname{age}(\gamma)-b_{1} \\
& \quad=\operatorname{codim}\left(\left(\mu_{\gamma} \circ p\right)_{\infty}\left(D_{w, \gamma, b_{1}, b_{2}, b_{3}, m_{1}, m_{2}, m_{3}}\right)\right)-\frac{m_{3}}{r} .
\end{aligned}
$$

Therefore, we obtain the desired formula

$$
\begin{aligned}
\operatorname{mld}_{x}\left(B, \mathfrak{a}^{\delta}\right) & =\inf _{w, m_{3}}\left\{\operatorname{codim}\left(\operatorname{Cont}^{w}(\mathfrak{a}) \cap \operatorname{Cont}^{m_{3}}\left(\mathfrak{n}_{r, B}\right) \cap \operatorname{Cont}^{\geqslant 1}\left(\mathfrak{m}_{x}\right)\right)-\frac{m_{3}}{r}-\delta w\right\} \\
& =\inf _{\gamma, w, b_{1}, b_{2}, b_{3}, m_{1}, m_{2}, m_{3}}\left\{\operatorname{codim}\left(\left(\mu_{\gamma} \circ p\right)_{\infty}\left(D_{w, \gamma, b_{1}, b_{2}, b_{3}, m_{1}, m_{2}, m_{3}}\right)\right)-\frac{m_{3}}{r}-\delta w\right\} \\
& =\inf _{\gamma, w, b_{1}, b_{2}, b_{3}, m_{1}, m_{2}, m_{3}}\left\{\operatorname{codim}\left(D_{w, \gamma, b_{1}, b_{2}, b_{3}, m_{1}, m_{2}, m_{3}}\right)+\operatorname{age}(\gamma)-b_{1}-\delta w\right\} \\
& =\inf _{\gamma, w, b_{1}}\left\{\operatorname{codim}\left(C_{w, \gamma, b_{1}}\right)+\operatorname{age}(\gamma)-b_{1}-\delta w\right\} .
\end{aligned}
$$

This completes the proof.

COROLlary 4.9. In the same setting as in Theorem 4.8, it follows that

$$
\operatorname{mld}_{x}\left(B, \mathfrak{a}^{\delta}\right)=\inf _{w, b_{1} \in \mathbb{Z}_{\geqslant 0}, \gamma \in G}\left\{\operatorname{codim}\left(C_{w, \gamma, b_{1}}^{\prime}\right)+\operatorname{age}(\gamma)-b_{1}-\delta w\right\}
$$

for

$$
C_{w, \gamma, b_{1}}^{\prime}=\operatorname{Cont}^{\geqslant w}\left(\mathfrak{a} \mathcal{O}_{\bar{B}^{(\gamma)}}\right) \cap \operatorname{Cont}^{\geqslant 1}\left(\mathfrak{m}_{x} \mathcal{O}_{\bar{B}^{(\gamma)}}\right) \cap \operatorname{Cont}^{b_{1}}\left(\operatorname{Jac}_{\bar{B}^{(\gamma)} / k[t]}\right) .
$$

Proof. Let $C_{w, \gamma, b_{1}}$ be the cylinder in Theorem 4.8. We fix $\gamma \in G$ and $b_{1} \in \mathbb{Z}_{\geqslant 0}$. Since we have $C_{w, \gamma, b_{1}} \subset C_{w, \gamma, b_{1}}^{\prime}$, it follows that

$$
\inf _{w}\left\{\operatorname{codim}\left(C_{w, \gamma, b_{1}}\right)-\delta w\right\} \geqslant \inf _{w}\left\{\operatorname{codim}\left(C_{w, \gamma, b_{1}}^{\prime}\right)-\delta w\right\} .
$$

We fix $w^{\prime} \in \mathbb{Z}_{\geqslant 0}$. Then it follows that

$$
\operatorname{codim}\left(C_{w^{\prime}, \gamma, b_{1}}^{\prime}\right)-\delta w^{\prime}=\min _{w \geqslant w^{\prime}}\left\{\operatorname{codim}\left(C_{w, \gamma, b_{1}}\right)\right\}-\delta w^{\prime} \geqslant \inf _{w}\left\{\operatorname{codim}\left(C_{w, \gamma, b_{1}}\right)-\delta w\right\} .
$$

The first equality follows from Proposition 2.25, and the last inequality follows from $\delta \geqslant 0$. Therefore, we have the opposite inequality

$$
\inf _{w}\left\{\operatorname{codim}\left(C_{w, \gamma, b_{1}}\right)-\delta w\right\} \leqslant \inf _{w}\left\{\operatorname{codim}\left(C_{w, \gamma, b_{1}}^{\prime}\right)-\delta w\right\} .
$$

This completes the proof.

Remark 4.10. Our formula can easily be extended to $\mathbb{R}$-ideals $\mathfrak{a}=\prod_{i=1}^{r} \mathfrak{a}_{i}^{\delta_{i}}$, where $\mathfrak{a}_{1}, \ldots, \mathfrak{a}_{r}$ are ideals and $\delta_{1}, \ldots, \delta_{r}$ are non-negative real numbers. In this setting, we have

$$
\begin{aligned}
\operatorname{mld}_{x}\left(B, \prod_{i=1}^{r} \mathfrak{a}_{i}^{\delta_{i}}\right) & =\inf _{w_{1}, \ldots, w_{r}, b_{1} \in \mathbb{Z}_{\geqslant 0}, \gamma \in G}\left\{\operatorname{codim}\left(C_{w_{1}, \ldots, w_{r}, \gamma, b_{1}}\right)+\operatorname{age}(\gamma)-b_{1}-\sum_{i=1}^{r} \delta_{i} w_{i}\right\} \\
& =\inf _{w_{1}, \ldots, w_{r}, b_{1} \in \mathbb{Z}_{\geqslant 0}, \gamma \in G}\left\{\operatorname{codim}\left(C_{w_{1}, \ldots, w_{r}, \gamma, b_{1}}^{\prime}\right)+\operatorname{age}(\gamma)-b_{1}-\sum_{i=1}^{r} \delta_{i} w_{i}\right\}
\end{aligned}
$$


for

$$
\begin{aligned}
& C_{w_{1}, \ldots, w_{r}, \gamma, b_{1}}=\left(\bigcap_{i=1}^{r} \operatorname{Cont}^{w_{i}}\left(\mathfrak{a}_{i} \mathcal{O}_{\bar{B}^{(\gamma)}}\right)\right) \cap \operatorname{Cont}^{\geqslant 1}\left(\mathfrak{m}_{x} \mathcal{O}_{\bar{B}^{(\gamma)}}\right) \cap \operatorname{Cont}^{b_{1}}\left(\operatorname{Jac}_{\bar{B}^{(\gamma)} / k[t]}\right), \\
& C_{w_{1}, \ldots, w_{r}, \gamma, b_{1}}^{\prime}=\left(\bigcap_{i=1}^{r} \operatorname{Cont}^{\geqslant w_{i}}\left(\mathfrak{a}_{i} \mathcal{O}_{\bar{B}^{(\gamma)}}\right)\right) \cap \operatorname{Cont}^{\geqslant 1}\left(\mathfrak{m}_{x} \mathcal{O}_{\bar{B}^{(\gamma)}}\right) \cap \operatorname{Cont}^{b_{1}}\left(\operatorname{Jac}_{\bar{B}^{(\gamma)} / k[t]}\right) \text {. }
\end{aligned}
$$

We state Theorem 4.8 in the case of quotient singularities.

Corollary 4.11. Let $x=0 \in A$ be the origin, and let $\mathfrak{a} \subset k\left[x_{1}, \ldots, x_{N}\right]^{G}$ be a non-zero ideal and $\delta$ be a non-negative real number. Then

$$
\operatorname{mld}_{x}\left(A, \mathfrak{a}^{\delta}\right)=\inf _{w \in \mathbb{Z}_{\geqslant 0}, \gamma \in G}\left\{\operatorname{codim}\left(C_{w, \gamma}\right)+\operatorname{age}(\gamma)-\delta w\right\}
$$

holds for

$$
C_{w, \gamma}=\operatorname{Cont}^{w}\left(\mathfrak{a} \mathcal{O}_{\bar{A}^{\prime}}\right) \cap \mathrm{Cont}^{\geqslant 1}\left(\mathfrak{m}_{x} \mathcal{O}_{\bar{A}^{\prime}}\right),
$$

where $\mathfrak{m}_{x} \subset \mathcal{O}_{A}$ is the maximal ideal corresponding to the closed point $x \in A$. Furthermore, the statements analogous to Corollary 4.9 and Remark 4.10 also hold.

Proof. Since $\bar{A}^{\prime}$ is smooth over $k[t]$, we have $\mathrm{Jac}_{\bar{A}^{\prime} / k[t]}=\mathcal{O}_{\bar{A}^{\prime}}$. Therefore, the assertion follows from Theorem 4.8 .

As a corollary, we obtain the following Reid-Tai-type formula on minimal log discrepancies. See also Remark 4.13 for a proof using the resolution of singularities.

Corollary 4.12 (cf. [Bor97, Question 2]). For $\gamma \in G$, we denote by $\langle\gamma\rangle$ the subgroup of $G$ generated by $\gamma$. Let $A^{\langle\gamma\rangle}=\mathbb{A}^{N} /\langle\gamma\rangle$, and let $x_{\gamma} \in A^{\langle\gamma\rangle}$ be the image of the origin of $\mathbb{A}^{N}$. Let $\mathfrak{a}$ be an $\mathbb{R}$-ideal sheaf on $A$. Then it follows that

$$
\operatorname{mld}_{x}(A, \mathfrak{a})=\min _{\gamma \in G} \operatorname{mld}_{x_{\gamma}}\left(A^{\langle\gamma\rangle}, \mathfrak{a} \mathcal{O}_{A\langle\gamma\rangle}\right) .
$$

Proof. We fix $\gamma \in G$ and $\gamma^{\prime} \in\langle\gamma\rangle$. Then we have a $k[t]$-ring homomorphism

$$
\bar{\lambda}_{\gamma^{\prime}}^{*}: k[t]\left[x_{1}, \ldots, x_{N}\right]^{G} \longrightarrow k[t]\left[x_{1}, \ldots, x_{N}\right]
$$

by applying the explanation in Subsection 4.1 to $G$ and $\gamma^{\prime}$. We also have a $k[t]$-ring homomorphism

$$
\bar{\lambda}_{\gamma^{\prime}}^{*}: k[t]\left[x_{1}, \ldots, x_{N}\right]^{\langle\gamma\rangle} \longrightarrow k[t]\left[x_{1}, \ldots, x_{N}\right]
$$

for $\langle\gamma\rangle$ and $\gamma^{\prime}$, where we use the same symbol $\bar{\lambda}_{\gamma^{\prime}}^{*}$ by abuse of notation. We note that, by definition, these two maps are compatible with the inclusion $k[t]\left[x_{1}, \ldots, x_{N}\right]^{G} \hookrightarrow k[t]\left[x_{1}, \ldots, x_{N}\right]^{\langle\gamma\rangle}$, which is induced by $k\left[x_{1}, \ldots, x_{N}\right]^{G} \hookrightarrow k\left[x_{1}, \ldots, x_{N}\right]^{\langle\gamma\rangle}$.

Let $\mathfrak{m}_{x} \subset k\left[x_{1}, \ldots, x_{N}\right]^{G}=\mathcal{O}_{A}$ and $\mathfrak{m}_{x_{\gamma}} \subset k\left[x_{1}, \ldots, x_{N}\right]^{\langle\gamma\rangle}=\mathcal{O}_{A\langle\gamma\rangle}$ be the maximal ideals corresponding to $x \in A$ and $x_{\gamma} \in A^{\langle\gamma\rangle}$, respectively. Since we have $\sqrt{\mathfrak{m}_{x} \mathcal{O}_{A\langle\gamma\rangle}}=\mathfrak{m}_{x_{\gamma}}$, it follows that

$$
\text { Cont }^{\geqslant 1}\left(\mathfrak{m}_{x} \mathcal{O}_{\bar{A}^{\prime}}\right)=\text { Cont }^{\geqslant 1}\left(\mathfrak{m}_{x_{\gamma}} \mathcal{O}_{\bar{A}^{\prime}}\right) \text {. }
$$

Therefore, $C_{w, \gamma^{\prime}}$ defined in Corollary 4.11 for $A, \gamma^{\prime} \in G$ and $\mathfrak{a}$ is exactly same as $C_{w, \gamma^{\prime}}$ defined for $A^{\langle\gamma\rangle}, \gamma^{\prime} \in\langle\gamma\rangle$ and $\mathfrak{a} \mathcal{O}_{A\langle\gamma\rangle}$. Hence the assertion

$$
\operatorname{mld}_{x}(A, \mathfrak{a})=\min _{\gamma \in G} \operatorname{mld}_{x_{\gamma}}\left(A^{\langle\gamma\rangle}, \mathfrak{a} \mathcal{O}_{A^{\langle\gamma\rangle}}\right)
$$

follows from Corollary 4.11. 


\section{Y. NAKAMURA AND K. ShibATA}

Remark 4.13. We can prove a more general statement following the argument in [Rei80] (cf. [Kol13, Section 2.42, Theorem 3.21]).

- Let $V$ be a $\mathbb{Q}$-Gorenstein normal variety, and let $G$ be a finite group acting on $V$. Let $x \in V$ be a closed point, and let $x^{\prime} \in V / G$ be its image. Let $V^{\langle\gamma\rangle}=V /\langle\gamma\rangle$, and let $x_{\gamma} \in V^{\langle\gamma\rangle}$ be the image of $x$. Let $\mathfrak{a}$ be an $\mathbb{R}$-ideal sheaf on $V / G$. Then it follows that

$$
\operatorname{mld}_{x^{\prime}}(V / G, \mathfrak{a})=\min _{\gamma \in G} \operatorname{mld}_{x_{\gamma}}\left(V^{\langle\gamma\rangle}, \mathfrak{a} \mathcal{O}_{V}\langle\gamma\rangle\right)
$$

By [Kol13, equation (2.42.4)], the inequality

$$
\operatorname{mld}_{x^{\prime}}(V / G, \mathfrak{a}) \leqslant \min _{\gamma \in G} \operatorname{mld}_{x_{\gamma}}\left(V^{\langle\gamma\rangle}, \mathfrak{a} \mathcal{O}_{V\langle\gamma\rangle}\right)
$$

is easy. Let $X^{\prime} \rightarrow X:=V / G$ be a resolution of singularities. We may assume that some divisor $E$ on $X^{\prime}$ computes $\operatorname{mld}_{x^{\prime}}(X, \mathfrak{a})$, that is, $E$ satisfies

- $c_{X}(E)=\left\{x^{\prime}\right\}$ and

- $\operatorname{mld}_{x^{\prime}}(X, \mathfrak{a})=a_{E}(X, \mathfrak{a})$ if $\operatorname{mld}_{x^{\prime}}(X, \mathfrak{a}) \geqslant 0$, and $a_{E}(X, \mathfrak{a})<0$ otherwise.

Let $V^{\prime}$ be the normalization of $X^{\prime}$ in the field of fractions $k(V)$ of $V$. Let $F$ be a divisor on $V^{\prime}$ which dominates $E$. We note that $G$ acts on $V^{\prime}$, and we have $X^{\prime}=V^{\prime} / G$. Let $G_{F}$ be the subgroup of $G$ that consists of an element $g \in G$ that fixes $F$ pointwise. Then by [Kol13, Equation (2.42.4)], we have

$$
a_{E}(V / G, \mathfrak{a})=a_{F^{\prime}}\left(V / G_{F}, \mathfrak{a} \mathcal{O}_{V / G_{F}}\right),
$$

where $F^{\prime}$ is the image of $F$ in $V / G_{F}$. Since $G_{F}$ is a cyclic group (cf. [IR96, Sections 2.5 and 2.6]), we get the opposite inequality.

As an application of Corollary 4.12, we can prove the ACC conjecture for quotient singularities (cf. [Mor20]).

Theorem 4.14. Let $n$ be a positive integer. The set

$$
A_{\text {quot }}(n):=\left\{\operatorname{mld}_{x}(X) \mid X \text { has a quotient singularity at } x \text { and } \operatorname{dim} X=n\right\}
$$

satisfies the ascending chain condition.

Proof. By Corollary 4.12 (or Remark 4.13), the set $A_{\text {quot }}(n)$ is equal to

$$
A_{\text {cquot }}(n):=\left\{\operatorname{mld}_{x}(X) \mid X \text { has a cyclic quotient singularity at } x \text { and } \operatorname{dim} X=n\right\} .
$$

Since a cyclic quotient singularity is a toric singularity (cf. [Rei87, Section (4.3)]), the set $A_{\text {cquot }}$ satisfies the ascending chain condition by [Amb06, Theorem 1]. This completes the proof.

\section{Precise inversion of the adjunction formula for quotient singularities}

In this section, we prove the precise inversion of the adjunction formula for the quotient of a complete intersection singularity by a finite linear group action (Theorem 5.1).

Let $\xi, G, \gamma, C_{\gamma}, Z, \bar{A}, A, A^{(\gamma)}, r, \bar{\lambda}_{\gamma}^{*}, \lambda_{\gamma}, q, \bar{\lambda}_{\gamma}, f_{i}, I, \widetilde{I}^{(\gamma)}, \bar{I}^{(\gamma)}, B, \widetilde{B}^{(\gamma)}, \bar{B}^{(\gamma)}, p, \tau, L_{\bar{B}^{(\gamma)}}$, $\mathfrak{n}_{1, p}^{\prime}$ and $\mathfrak{n}_{1, \bar{\mu}_{\gamma}}^{\prime}$ be as in Section 4 .

TheOREM 5.1. Let $\mathfrak{a} \subset k\left[x_{1}, \ldots, x_{N}\right]^{G}$ be a non-zero ideal, and let $\delta$ be a non-negative real number. We set $\mathfrak{b}=\mathfrak{a}\left(k\left[x_{1}, \ldots, x_{N}\right]^{G} /\left(f_{1}, \ldots, f_{c}\right)\right)$. Suppose $\mathfrak{b} \neq 0$. Let $x=0 \in A$ be the origin. 


\section{INVERSION OF ADJUNCTION FOR QUOTIENT SINGULARITIES}

Suppose that $B$ is klt. Then we have

$$
\operatorname{mld}_{x}\left(A,\left(f_{1} \cdots f_{c}\right) \mathfrak{a}^{\delta}\right)=\operatorname{mld}_{x}\left(B, \mathfrak{b}^{\delta}\right) .
$$

Proof. Since $\operatorname{mld}_{x}\left(A,\left(f_{1} \cdots f_{c}\right) \mathfrak{a}^{\delta}\right) \leqslant \operatorname{mld}_{x}\left(B, \mathfrak{b}^{\delta}\right)$ is true in general by adjunction, it is enough to prove the opposite inequality $\operatorname{mld}_{x}\left(A,\left(f_{1} \cdots f_{c}\right) \mathfrak{a}^{\delta}\right) \geqslant \operatorname{mld}_{x}\left(B, \mathfrak{b}^{\delta}\right)$.

By Corollary 4.9 (cf. Remark 4.10), we have

$$
\operatorname{mld}_{x}\left(A,\left(f_{1} \cdots f_{c}\right) \mathfrak{a}^{\delta}\right)=\inf _{w, v \in \mathbb{Z} \geqslant 0, \gamma \in G}\left\{\operatorname{codim}_{\bar{A}_{\infty}^{\prime}}\left(C_{w, v, \gamma}\right)+\operatorname{age}(\gamma)-w-\delta v\right\}
$$

where we define $C_{w, v, \gamma} \subset \bar{A}_{\infty}^{\prime}$ as

$$
C_{w, v, \gamma}:=\text { Cont }^{\geqslant w}\left(f_{1} \cdots f_{c} \mathcal{O}_{\bar{A}^{\prime}}\right) \cap \mathrm{Cont}^{\geqslant v}\left(\mathfrak{a} \mathcal{O}_{\bar{A}^{\prime}}\right) \cap \text { Cont }^{\geqslant 1}\left(\mathfrak{m}_{x} \mathcal{O}_{\bar{A}^{\prime}}\right) .
$$

We fix $w, v \in \mathbb{Z}_{\geqslant 0}$ and $\gamma \in G$.

First, we note that the following claim holds. It will be proved in the end of the proof of Theorem 5.1.

Claim 5.2. The intersection $C^{\prime} \cap \bar{B}_{\infty}^{(\gamma)}$ is not a thin set of $\bar{B}_{\infty}^{(\gamma)}$ for any irreducible component $C^{\prime}$ of $C_{w, v, \gamma}$.

Let $C_{w, v, \gamma}^{\prime} \subset C_{w, v, \gamma}$ be an irreducible component satisfying

$$
\operatorname{codim}_{\bar{A}_{\infty}^{\prime}}\left(C_{w, v, \gamma}\right)=\operatorname{codim}_{\bar{A}_{\infty}^{\prime}}\left(C_{w, v, \gamma}^{\prime}\right) .
$$

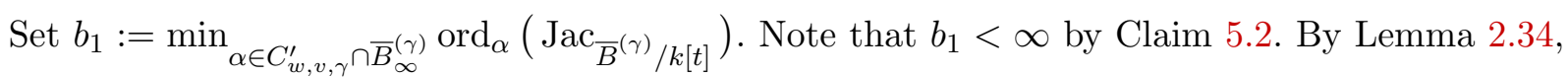
we have

$$
\begin{aligned}
& \operatorname{codim}_{\bar{A}_{\infty}^{\prime}}\left(C_{w, v, \gamma}^{\prime} \cap \operatorname{Cont}^{\leqslant b_{1}}\left(\left(\tau^{*}\right)^{-1} \operatorname{Jac}_{\bar{B}^{(\gamma)} / k[t]}\right)\right)-w \\
& \geqslant \operatorname{codim}_{\bar{B}_{\infty}^{(\gamma)}}\left(C_{w, v, \gamma}^{\prime} \cap \bar{B}_{\infty}^{(\gamma)} \cap \operatorname{Cont}^{b_{1}}\left(\operatorname{Jac}_{\bar{B}^{(\gamma)} / k[t]}\right)\right)-b_{1} .
\end{aligned}
$$

Since $C_{w, v, \gamma}^{\prime}$ is an irreducible closed cylinder, its non-empty open subcylinder has the same codimension. Therefore,

$$
\operatorname{codim}_{\bar{A}_{\infty}^{\prime}}\left(C_{w, v, \gamma}^{\prime} \cap \operatorname{Cont}^{\leqslant b_{1}}\left(\left(\tau^{*}\right)^{-1} \operatorname{Jac}_{\bar{B}^{(\gamma)} / k[t]}\right)\right)=\operatorname{codim}_{\bar{A}_{\infty}^{\prime}}\left(C_{w, v, \gamma}^{\prime}\right) .
$$

Hence we have

$$
\operatorname{codim}_{\bar{A}_{\infty}^{\prime}}\left(C_{w, v, \gamma}\right)-w \geqslant \operatorname{codim}_{\bar{B}_{\infty}^{(\gamma)}}\left(C_{w, v, \gamma} \cap \bar{B}_{\infty}^{(\gamma)} \cap \operatorname{Cont}^{b_{1}}\left(\operatorname{Jac}_{\bar{B}^{(\gamma)} / k[t]}\right)\right)-b_{1} .
$$

Since

$$
\begin{aligned}
& C_{w, v, \gamma} \cap \bar{B}_{\infty}^{(\gamma)} \cap \operatorname{Cont}^{b_{1}}\left(\operatorname{Jac}_{\bar{B}^{(\gamma)} / k[t]}\right) \\
& \quad \subset \operatorname{Cont}^{\geqslant v}\left(\mathfrak{b}_{\bar{B}^{(\gamma)}}\right) \cap \operatorname{Cont}^{\geqslant 1}\left(\mathfrak{m}_{x} \mathcal{O}_{\bar{B}^{(\gamma)}}\right) \cap \operatorname{Cont}^{b_{1}}\left(\operatorname{Jac}_{\bar{B}^{(\gamma)} / k[t]}\right),
\end{aligned}
$$

by applying Corollary 4.9 (cf. Remark 4.10) to $B$, we obtain the desired formula

$$
\begin{aligned}
& \operatorname{mld}_{x}\left(A,\left(f_{1} \cdots f_{c}\right) \mathfrak{a}^{\delta}\right) \\
& =\inf _{w, v \in \mathbb{Z}_{\geqslant 0}, \gamma \in G}\left\{\operatorname{codim}_{\bar{A}_{\infty}^{\prime}}\left(C_{w, v, \gamma}\right)+\operatorname{age}(\gamma)-w-\delta v\right\} \\
& \geqslant \inf _{v, b \in \mathbb{Z}_{\geqslant 0}, \gamma \in G}\left\{\operatorname{codim}_{\bar{B}_{\infty}^{(\gamma)}}\left(\begin{array}{c}
\mathrm{Cont}^{\geqslant v}\left(\mathfrak{b O}_{\left.\bar{B}^{(\gamma)}\right)} \cap \operatorname{Cont}^{\geqslant 1}\left(\mathfrak{m}_{x} \mathcal{O}_{\bar{B}^{(\gamma)}}\right)\right. \\
\cap \operatorname{Cont}^{b}\left(\operatorname{Jac}_{\bar{B}^{(\gamma)} / k[t]}\right)
\end{array}\right)-b-\delta v\right\} \\
& =\operatorname{mld}_{x}\left(B, \mathfrak{b}^{\delta}\right) \text {. }
\end{aligned}
$$




\section{Y. NAKAmura AND K. Shibata}

Therefore, it is sufficient to show Claim 5.2.

Proof of Claim 5.2. First, we introduce some notation. For an $\operatorname{arc} \alpha \in \bar{A}_{\infty}^{\prime}$, we set $g_{i}^{\alpha}:=\alpha^{*}\left(x_{i}\right) \in$ $k[[t]]$, where $\alpha^{*}: k[t]\left[x_{1}, \ldots, x_{N}\right] \rightarrow k[[t]]$ is the corresponding ring homomorphism. We denote by $\beta \in \bar{A}_{\infty}^{\prime}$ the trivial arc determined by $g_{i}^{\beta}:=0$. Let $T \subset \bar{A}^{\prime}$ be the closed subscheme defined by the ideal $\left(x_{1}, \ldots, x_{N}\right) \subset k[t]\left[x_{1}, \ldots, x_{N}\right]$. Then we have $T_{\infty}=\{\beta\}$.

Since $\bar{I}^{(\gamma)} \subset\left(x_{1}, \ldots, x_{N}\right)$, it follows that $\beta \in T_{\infty} \subset \bar{B}_{\infty}^{(\gamma)}$. Let $W \subset \bar{B}^{(\gamma)}$ be the irreducible component dominating Spec $k[t]$, and let $h: W^{\prime} \rightarrow W$ be a resolution of singularities of $W$. Let $T^{\prime}:=h^{-1}(T)$. Since $B$ is klt by assumption, we note that $\bar{B}$ is also klt by Lemma 4.2 . Therefore, $W$ is klt outside $t=0$ by Remark 4.3, and hence $T^{\prime} \rightarrow T$ has a section by [HM07, Corollary 1.7(2)]. Consequently, there exists an arc $\beta^{\prime} \in T_{\infty}^{\prime} \subset W_{\infty}^{\prime}$ such that $h_{\infty}\left(\beta^{\prime}\right)=\beta$.

Then by Lemma 2.27, it is suffices to show that $\beta \in C^{\prime}$. In order to prove this, we introduce a $k$-action on the arc space $\bar{A}_{\infty}^{\prime}$ as follows. Let $\alpha \in \bar{A}_{\infty}^{\prime}$ and $a \in k$. Then we define $a \cdot \alpha$ by

$$
g_{i}^{a \cdot \alpha}(t):=a^{e_{i}} g_{i}^{\alpha}\left(a^{d} t\right)
$$

We adopt the convention that $a^{e_{i}}=1$ when $a=0$ and $e_{i}=0$. Then for $f \in k\left[x_{1}, \ldots, x_{N}\right]^{G}$, it is easy to see that $v(t)=u\left(a^{d} t\right)$ when we set

$$
u(t):=\alpha^{*}\left(\bar{\lambda}_{\gamma}^{*}(f)\right), \quad v(t):=(a \cdot \alpha)^{*}\left(\bar{\lambda}_{\gamma}^{*}(f)\right) \in k[[t]]
$$

for $\alpha \in \bar{A}_{\infty}^{\prime}$ and $a \in k$. Therefore,

$$
\operatorname{ord}_{\alpha}\left(\bar{\lambda}_{\gamma}^{*}(f)\right)=\operatorname{ord}_{a \cdot \alpha}\left(\bar{\lambda}_{\gamma}^{*}(f)\right)
$$

holds if $a \in k^{\times}$. Hence any cylinder of form Cont ${ }^{\geqslant c}\left(\mathfrak{c} \mathcal{O}_{\bar{A}^{\prime}}\right)$ with an ideal $\mathfrak{c} \subset k\left[x_{1}, \ldots, x_{N}\right]^{G}$ is invariant under the $k$-action. Therefore, $C_{w, v, \gamma}$ and its irreducible component $C^{\prime}$ are invariant under the $k$-action. The assertion $\beta \in C^{\prime}$ then follows from the observation that $\beta=0 \cdot \alpha$ holds for any $\alpha \in$ Cont $^{\geqslant 1}\left(\mathfrak{m}_{x} \mathcal{O}_{\bar{B}}^{(\gamma)}\right)$.

This completes the proof of the theorem.

Remark 5.3. In Theorem 5.1, we assume that $B$ is klt. This assumption is essential in the proof of Claim 5.2.

If $B$ is not klt, then the arc space $\bar{B}_{\infty}^{(\gamma)}$ itself might be a thin set of $\bar{B}_{\infty}^{(\gamma)}$. Let

$$
\bar{B}=\operatorname{Spec} k\left[x_{1}, x_{2}, x_{3}\right] /\left(x_{1}^{3}+x_{2}^{3}+x_{3}^{3}\right), \quad\left(d, e_{1}, e_{2}, e_{3}\right)=(3,0,1,2) .
$$

Then we have

$$
\bar{B}^{(\gamma)}=\operatorname{Spec} k[t]\left[x_{1}, x_{2}, x_{3}\right] /\left(x_{1}^{3}+t x_{2}^{3}+t^{2} x_{3}^{3}\right),
$$

and it follows that $\bar{B}_{\infty}^{(\gamma)}=\{\beta\}$, where $\beta$ is the trivial arc corresponding to the origin. Therefore, $\bar{B}_{\infty}^{(\gamma)}$ turns out to be a thin set of $\bar{B}_{\infty}^{(\gamma)}$.

Remark 5.4. Theorem 5.1 can be generalized to $\mathbb{R}$-ideals due to Remark 4.10. Let $\mathfrak{a}$ be an $\mathbb{R}$-ideal on $A$. Then we have

$$
\operatorname{mld}_{x}\left(A,\left(f_{1} \cdots f_{c}\right) \mathfrak{a}\right)=\operatorname{mld}_{x}\left(B, \mathfrak{a} \mathcal{O}_{B}\right) .
$$

Remark 5.5. All the statements in Section 4 are still true when we replace $k\left[x_{1}, \ldots, x_{N}\right]$ with its localization $k\left[x_{1}, \ldots, x_{N}\right]_{g}$ at a $G$-invariant element $g$ which does not vanish at the origin. Therefore, Theorem 5.1 is also true for the local setting, that is, when $B$ is locally defined by a regular sequence $f_{1}, \ldots, f_{c}$ at the origin and has only klt singularity at the origin. 


\section{INVERSION OF ADJUNCTION FOR QUOTIENT SINGULARITIES}

\section{Proofs of the main theorems}

As a corollary of Theorem 5.1, we prove the PIA conjecture for hyperquotient singularities.

Corollary 6.1. Suppose that a finite subgroup $G \subset \mathrm{GL}_{N}(k)$ acts on $\mathbb{A}_{k}^{N}$ freely in codimension one. Let $X:=\mathbb{A}_{k}^{N} / G$ be the quotient variety, and let $x \in X$ be the image of the origin of $\mathbb{A}_{k}^{N}$. Let $Y$ be a subvariety of $X$ through $x$ of codimension $c$, and let $\mathfrak{a}$ be an $\mathbb{R}$-ideal sheaf on $Y$. Suppose that $Y$ is locally defined by $c$ equations at $x$ in $X$. Let $D$ be a Cartier prime divisor on $Y$ through $x$ with a klt singularity at $x \in D$. Suppose that $D$ is not contained in the cosupport of the $\mathbb{R}$-ideal sheaf $\mathfrak{a}$. Then it follows that

$$
\operatorname{mld}_{x}\left(Y, \mathfrak{a} \mathcal{O}_{Y}(-D)\right)=\operatorname{mld}_{x}\left(D, \mathfrak{a} \mathcal{O}_{D}\right) .
$$

Proof. Let $R:=k\left[x_{1}, \ldots, x_{N}\right]^{G}$ be the invariant ring. Take an $\mathbb{R}$-ideal sheaf $\mathfrak{b}$ on $X$ such that $\mathfrak{a}=\mathfrak{b} \mathcal{O}_{Y}$, and take local equations $f_{1}, \ldots, f_{c} \in \mathcal{O}_{X, x}$ of $Y$ in $X$. Furthermore, take $g \in \mathcal{O}_{X, x}$ such that its image $\bar{g} \in \mathcal{O}_{Y, x}$ defines $D$. We note that $Y$ has a klt singularity at $x$ by inversion of adjunction (cf. [KM98, Theorem 5.50]). Then it follows that

$$
\operatorname{mld}_{x}\left(Y, \mathfrak{a} \mathcal{O}_{Y}(-D)\right)=\operatorname{mld}_{x}\left(X,\left(f_{1} \cdots f_{c} \cdot g\right) \mathfrak{b}\right)=\operatorname{mld}_{x}\left(D, \mathfrak{a} \mathcal{O}_{D}\right)
$$

by applying Theorem 5.1 twice (cf. Remark 5.5).

Theorem 6.2. Suppose that a finite subgroup $G \subset \mathrm{GL}_{N}(k)$ acts on $\mathbb{A}_{k}^{N}$ freely in codimension one. Let $X:=\mathbb{A}_{k}^{N} / G$ be the quotient variety. Let $Y$ be a subvariety of $X$ of codimension $c$ which has only klt singularities, and let $\mathfrak{a}$ be an $\mathbb{R}$-ideal sheaf on $Y$. Suppose that $Y$ is locally defined by $c$ equations in $X$. Then the function

$$
|Y| \rightarrow \mathbb{R}_{\geqslant 0} \cup\{-\infty\}, \quad y \mapsto \operatorname{mld}_{y}(Y, \mathfrak{a})
$$

is lower semi-continuous, where we denote by $|Y|$ the set of all closed points of $Y$ with the Zariski topology.

Proof. We keep the notation of the proof of Corollary 6.1. Since the lower semi-continuity holds for $X$ by [Nak16b, Corollary 1.3], it is sufficient to show that

$$
\operatorname{mld}_{y}(Y, \mathfrak{a})=\operatorname{mld}_{y}\left(X,\left(f_{1} \cdots f_{c}\right) \mathfrak{b}\right)
$$

for any $y \in Y$.

We fix a closed point $y \in Y$. Take a closed point $y^{\prime} \in \mathbb{A}_{k}^{N}$ whose image in $Y$ is $y$. Let $G_{y^{\prime}}:=\left\{g \in G \mid g\left(y^{\prime}\right)=y^{\prime}\right\}$ be the stabilizer group of $y^{\prime}$. Then $\mathbb{A}^{N} / G_{y^{\prime}} \rightarrow \mathbb{A}^{N} / G$ is étale at $y$. We note that the minimal log discrepancy is preserved under an étale map. Hence by replacing $X=\mathbb{A}_{k}^{N} / G$ with $\mathbb{A}^{N} / G_{y^{\prime}}$ and changing the coordinate of $\mathbb{A}^{N}$, we may assume that $y^{\prime}$ is the origin and the group action is still linear. Then we have

$$
\operatorname{mld}_{y}(Y, \mathfrak{a})=\operatorname{mld}_{y}\left(X,\left(f_{1} \cdots f_{c}\right) \mathfrak{b}\right)
$$

by Theorem 5.1, which completes the proof.

\section{ACKNOWLEDGEMENTS}

We would like to thank Professor Takehiko Yasuda for discussions and many suggestions. We also thank the referee, whose comments and suggestions have greatly improved the article. The referee pointed out the relevance to the papers [Seb04, Yas16, Yas19]. Remarks 2.14 and 3.9 are largely due to the referee's comments. 


\section{Y. NAKAMURA AND K. ShiBATA}

\section{REFERENCES}

Amb99 F. Ambro, On minimal log discrepancies, Math. Res. Lett. 6 (1999), no. 5, 573-580; doi: 10.4310/MRL.1999.v6.n5.a10.

Amb06 - The set of toric minimal log discrepancies, Cent. Eur. J. Math. 4 (2006), no. 3, 358-370; doi:10.2478/s11533-006-0013-x.

Bor97 A. Borisov, Minimal discrepancies of toric singularities, Manuscripta Math. 92 (1997), no. 1, 33-45; doi:10.1007/BF02678179.

CNS18 A. Chambert-Loir, J. Nicaise and J. Sebag, Motivic integration, Progr. Math., vol. 325 (Birkhäuser/Springer, New York, 2018); doi:10.1007/978-1-4939-7887-8.

DL99 J. Denef and F. Loeser, Germs of arcs on singular algebraic varieties and motivic integration, Invent. Math. 135 (1999), no. 1, 201-232; doi:10.1007/s002220050284.

DL02 Motivic integration, quotient singularities and the McKay correspondence, Compos. Math. 131 (2002), no. 3, 267-290; doi:10.1023/A:1015565912485.

Eis95 D. Eisenbud, Commutative algebra with a view toward algebraic geometry, Grad. Texts in Math., vol. 150 (Springer-Verlag, New York, 1995); doi:10.1007/978-1-4612-5350-1.

EM04 L. Ein and M. Mustaţă, Inversion of adjunction for local complete intersection varieties, Amer. J. Math. 126 (2004), no. 6, 1355-1365; doi:10.1353/ajm.2004.0044.

EM09 Jet schemes and singularities, in Algebraic Geometry - Seattle 2005, Part 2, Proc. Sympos. Pure Math., vol. 80 (Amer. Math. Soc., Providence, RI, 2009), 505-546; doi:10. $1090 /$ pspum/080.2/2483946.

EMY03 L. Ein, M. Mustaţă and T. Yasuda, Jet schemes, log discrepancies and inversion of adjunction, Invent. Math. 153 (2003), no. 3, 519-535; doi:10.1007/s00222-003-0298-3.

GHS03 T. Graber, J. Harris and J. Starr, Families of rationally connected varieties, J. Amer. Math. Soc. 16 (2003), no. 1, 57-67; doi:10.1090/S0894-0347-02-00402-2.

GW10 U. Görtz and T. Wedhorn, Algebraic geometry I. schemes with examples and exercises, Adv. Lectures Math. (Vieweg + Teubner, Wiesbaden, 2010); doi:10.1007/978-3-8348-9722-0.

Har77 R. Hartshorne, Algebraic geometry, Grad. Texts in Math., vol. 52 (Springer-Verlag, New York Heidelberg, 1977); doi:10.1007/978-1-4757-3849-0.

HLS19 J. Han, J. Liu and V.V. Shokurov, ACC for minimal log discrepancies of exceptional singularities, 2019, arXiv: 1903.04338.

HM07 C. D. Hacon and J. Mckernan, On Shokurov's rational connectedness conjecture, Duke Math. J. 138 (2007), no. 1, 119-136; doi:10.1215/S0012-7094-07-13813-4.

IR96 Y. Ito and M. Reid, The McKay correspondence for finite subgroups of SL(3, C), HigherDimensional Complex Varieties (Trento, 1994) (de Gruyter, Berlin, 1996), 221-240; doi: 10.1515/9783110814736.221.

Kaw14 M. Kawakita, Discreteness of log discrepancies over log canonical triples on a fixed pair, J. Algebraic Geom. 23 (2014), no. 4, 765-774; doi:10.1090/S1056-3911-2014-00630-5.

Kaw15_, A connectedness theorem over the spectrum of a formal power series ring, Internat. J. Math. 26 (2015), no. 11, 1550088; doi:10.1142/S0129167X15500883.

Kaw21_, On equivalent conjectures for minimal log discrepancies on smooth threefolds, J. Algebraic Geom. 30 (2021), no. 1, 97-149; doi:10.1090/jag/757.

KM98 J. Kollár and S. Mori, Birational geometry of algebraic varieties, Cambridge Tracts in Math., vol. 134 (Cambridge Univ. Press, Cambridge, 1998); doi:10.1017/CB09780511662560.

Kol92 J. Kollár (ed.), Flips and abundance for algebraic threefolds, a summer seminar at the University of Utah (Salt Lake City, 1991), Astérisque, 211 (1992).

Kol07_ Lectures on resolution of singularities, Annals of Mathematics Studies, vol. 166, Princeton University Press, Princeton, NJ, 2007. 


\section{INVERSION OF ADJUNCTION FOR QUOTIENT SINGULARITIES}

Kol13_ Singularities of the minimal model program, Cambridge Tracts in Math., vol. 200 (Cambridge Univ. Press, Cambridge, 2013); doi:10.1017/CB09781139547895.

Liu02 Q. Liu, Algebraic geometry and arithmetic curves, Oxford Grad. Texts Math., vol. 6 (Oxford Univ. Press, Oxford, 2002).

Mat89 H. Matsumura, Commutative ring theory, 2nd ed., Cambridge Stud. Adv. Math., vol. 8 (Cambridge Univ. Press, Cambridge, 1989).

MN18 M. Mustaţă and Y. Nakamura, A boundedness conjecture for minimal log discrepancies on a fixed germ, in Local and Global Methods in Algebraic Geometry, Contemp. Math., vol. 712 (Amer. Math. Soc., Providence, RI, 2018), 287-306; doi:10.1090/conm/712/14351.

Mor20 J. Moraga, Small quotient minimal log discrepancies, 2020, arXiv:2008.13311.

Nag59 M. Nagata, On the purity of branch loci in regular local rings, Illinois J. Math. 3 (1959), 328-333; doi:10.1215/ijm/1255455255.

Nak16a Y. Nakamura, On minimal log discrepancies on varieties with fixed Gorenstein index, Michigan Math. J. 65 (2016), no. 1, 165-187; doi:10.1307/mmj/1457101816.

Nak16b_, On semi-continuity problems for minimal log discrepancies, J. reine angew. Math. 711 (2016), 167-187; doi:10.1515/crelle-2013-0112.

Rei80 M. Reid, Canonical 3-folds, Journées de Géometrie Algébrique d'Angers, Juillet 1979/Algebraic Geometry, Angers, 1979 (Sijthoff \& Noordhoff, Alphen aan den Rijn - Germantown, MD, 1980), 273-310.

Rei87 _ Y Young person's guide to canonical singularities, Algebraic Geometry, Bowdoin, 1985 (Brunswick, Maine, 1985), Proc. Sympos. Pure Math., vol. 46 (Amer. Math. Soc., Providence, RI, 1987), 345-414.

Seb04 J. Sebag, Intégration motivique sur les schémas formels, Bull. Soc. Math. France 132 (2004), no. 1, 1-54; doi:10.24033/bsmf. 2458.

Shi19 K. Shibata, Minimal log discrepancies in positive characteristic, Comm. Algebra 50 (2022), no. 2, 571-582; doi:10.1080/00927872.2021.1962898.

Sho04 V.V. Shokurov, Letters of a bi-rationalist. V. Minimal log discrepancies and termination of log flips, Proc. Steklov Inst. Math. 246 (2004), no. 3, 315-336.

Sta21 The Stacks Project Authors, Stacks Project, version 2021, available at https://stacks.math. columbia.edu.

WY15 M. M. Wood and T. Yasuda, Mass formulas for local Galois representations and quotient singularities. I: a comparison of counting functions, Int. Math. Res. Not. 2015 (2015), no. 23, 12590-12619; doi:10.1093/imrn/rnv074.

Yas04 T. Yasuda, Twisted jets, motivic measures and orbifold cohomology, Compos. Math. 140 (2004), no. 2, 396-422; doi:10.1112/S0010437X03000368.

Yas06 _ Motivic integration over Deligne-Mumford stacks, Adv. Math. 207 (2006), no. 2, 707761; doi:10.1016/j.aim.2006.01.004.

Yas16 W Wilder McKay correspondences, Nagoya Math. J. 221 (2016), no. 1, 111-164; doi: 10.1017/nmj.2016.3.

Yas19__ Motivic integration over wild Deligne-Mumford stacks, 2019, arXiv:1908.02932.

Yusuke Nakamura nakamura@ms.u-tokyo.ac.jp

Graduate School of Mathematical Sciences, the University of Tokyo, 3-8-1 Komaba, Meguro-ku, Tokyo 153-8914, Japan

Kohsuke Shibata shibata.kohsuke@gmail.com

Department of Mathematics, College of Humanities and Sciences, Nihon University, 3-25-40 Sakurajosui, Setagaya-ku, Tokyo 156-8550, Japan 